\title{
Synthesis of Aminated Phenanthridinones via Palladium/Norbornene Catalysis
}

\author{
Xavier Abel-Snape, Andrew Whyte, and Mark Lautens* \\ mark.lautens@utoronto.ca
}
Department of Chemistry, University of Toronto, 80 St. George Street, Toronto, Ontario, M5S 3H6, Canada

\section{Supporting Information}

\section{Table of Contents}

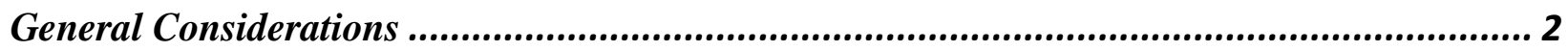

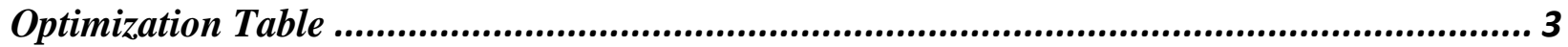

Procedures for the Synthesis of Substrates............................................................................... 4

General Procedure 1 (GP1) ................................................................................................

General Procedure 2 (GP2) .......................................................................................................4

Procedures for the Catalytic Reactions .................................................................................. 6

General Procedure 3 (GP3) .............................................................................................

General Procedure 4 (GP4) ................................................................................................

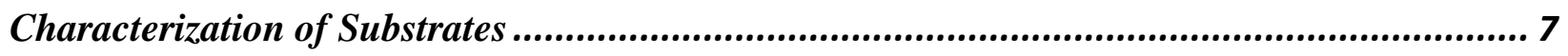

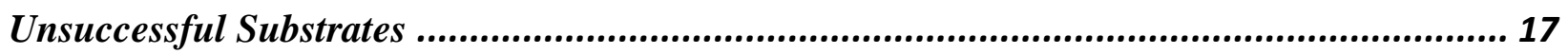

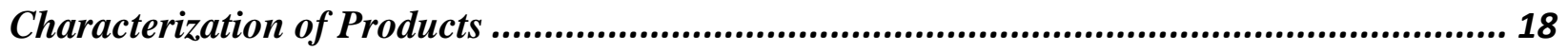

Procedure for the 1 mmol-Scale Synthesis of $3 j$................................................................... 33

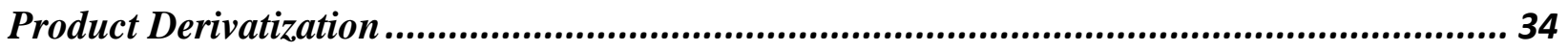

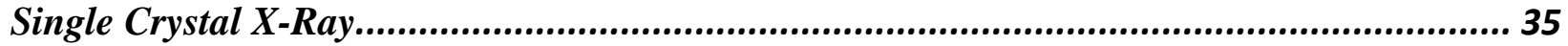

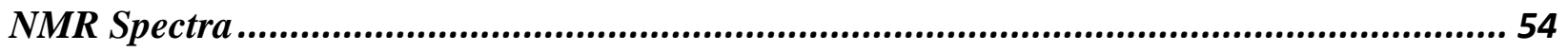

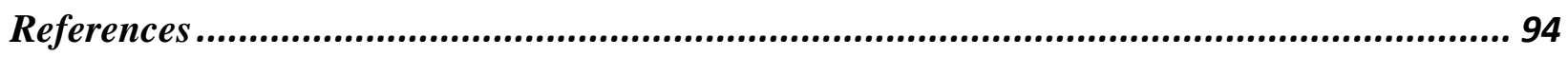




\section{General Considerations}

Reactions were performed under argon in a dry environment unless otherwise stated. Reaction progress was monitored by thin layer chromatography (TLC) and visualized under UV light. Toluene was distilled over calcium hydride. $\mathrm{Pd}\left(\mathrm{PPh}_{3}\right)_{4}$ was purchased from Sigma Aldrich and kept in the freezer. Potassium carbonate (anhydrous) was purchased from Sigma Aldrich and was stored in a desiccator. 1,3,5Trimethoxybenzene was purchased from Combi-Blocks and used as received. All other starting materials and reagents were purchased from Sigma, Alfa Aesar, Fisher, or Combi-Blocks and were used as received. Catalytic reactions were performed in 2 dram vials equipped with a teflon septa (ThermoScientific National B7995-15) and a stir bar (Fisher cat no. 14-513-57, 12 x 4.5 mm). An oil bath was used as the heating source for reactions requiring heat. Flash column chromatography was performed with Silicycle $46-60 \mu \mathrm{m}$ silica gel. $O$-benzoylhydroxylamines were synthesized following previous methods. ${ }^{1}$

${ }^{1} \mathrm{H}$ and ${ }^{13} \mathrm{C}$ NMR were obtained at $296 \mathrm{~K}$ on Agilent DD2 500 equipped with a $5 \mathrm{~mm}$ Xses Cold Probe. Measurements were referenced to the solvent. NMR data are referenced as chemical shift ( $\delta \mathrm{ppm})$, multiplicity ( $\mathrm{s}=$ singlet, $\mathrm{d}=$ doublet, $\mathrm{t}=$ triplet, $\mathrm{q}=$ quartet, $\mathrm{m}=$ multiplet, $\mathrm{b}=$ broad), coupling constant $(\mathrm{Hz})$, integration. NMR yields were obtained by ${ }^{1} \mathrm{H}$ NMR analysis using a 10 second relaxation delay and 1,3,5-trimethoxybenzene as an internal standard. HRMS were obtained on a JEOL AccuTOF-DART performed at the Advanced Instrumentation for Molecular Structure (AIMS) at the University of Toronto. IR spectra were aquired on a Perkin-Elmer Spectrum 100 instrument with a single-bounce diamond/ZeSe ATR accessory. Data is presented in wavenumbers $\left(\mathrm{cm}^{-1}\right)$. 


\section{Optimization Table}

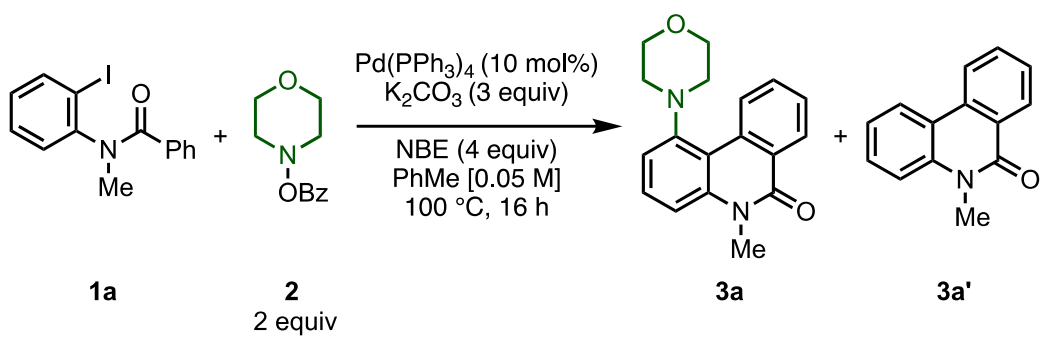

\begin{tabular}{|c|c|c|c|}
\hline entry & variation(s) & $\mathbf{3 a}(\%)^{\mathrm{a}}$ & $3 \mathbf{a}^{\prime}(\%)^{\mathrm{a}}$ \\
\hline 1 & none & 77 & 0 \\
\hline 2 & $\mathrm{Cs}_{2} \mathrm{CO}_{3}$ instead of $\mathrm{K}_{2} \mathrm{CO}_{3}$, PivOH as additive (30 mol\%) & 50 & 37 \\
\hline 3 & $\mathrm{Cs}_{2} \mathrm{CO}_{3}$ instead of $\mathrm{K}_{2} \mathrm{CO}_{3}, 120{ }^{\circ} \mathrm{C}$ & 63 & 18 \\
\hline 4 & $\mathrm{Cs}_{2} \mathrm{CO}_{3}$ instead of $\mathrm{K}_{2} \mathrm{CO}_{3}, 80{ }^{\circ} \mathrm{C}$ & 53 & 31 \\
\hline 5 & $\mathrm{Cs}_{2} \mathrm{CO}_{3}$ instead of $\mathrm{K}_{2} \mathrm{CO}_{3}$ & 77 & 4 \\
\hline 6 & $\mathrm{PivOH}$ as additive (30 mol\%) & 62 & 1 \\
\hline 7 & $120^{\circ} \mathrm{C}$ & 75 & 0 \\
\hline 8 & $0.1 \mathrm{M}$ & 68 & 0 \\
\hline 9 & $\mathrm{Pd}(\mathrm{OAc})_{2}(10 \mathrm{~mol} \%), \mathrm{PPh}_{3}(20 \mathrm{~mol} \%)$ & 56 & 2 \\
\hline 10 & $\mathrm{Pd}(\mathrm{OAc})_{2}(10 \mathrm{~mol} \%), \mathrm{P}(\mathrm{PMP})_{3}(20 \mathrm{~mol} \%)$ & 55 & 2 \\
\hline 11 & $\operatorname{Pd}(\mathrm{OAc})_{2}(10 \mathrm{~mol} \%)$, JohnPhos (20 mol\%) & 8 & 0 \\
\hline 12 & $\mathrm{~K}_{2} \mathrm{CO}_{3}$ (2 equiv) & 58 & 0 \\
\hline 13 & $\mathrm{~K}_{2} \mathrm{CO}_{3}$ (4 equiv) & 75 & 0 \\
\hline 14 & NBE (3 equiv) & 62 & 0 \\
\hline 15 & NBE (5 equiv) & 58 & 0 \\
\hline 16 & THF instead of PhMe & 47 & 0 \\
\hline 17 & $\mathrm{MeCN}$ instead of $\mathrm{PhMe}$ & 35 & 1 \\
\hline 18 & 1,4-dioxane instead of $\mathrm{PhMe}$ & 61 & 0 \\
\hline 19 & $\mathrm{Pd}\left(\mathrm{PPh}_{3}\right)_{4} 5 \mathrm{~mol} \%$ & 75 & 0 \\
\hline $20^{b}$ & $\operatorname{Pd}\left(\mathbf{P P h}_{3}\right)_{4} 5$ mol\%, 2 (2 equiv) & $79(74)^{b}$ & $\mathbf{0}$ \\
\hline
\end{tabular}

${ }^{a}$ Yields were determined by ${ }^{1} \mathrm{H}$ NMR spectroscopy analysis of crude reaction mixture using 1,3,5-trimethoxybenzene as an internal standard. 'bsolated yield. 


\section{Procedures for the Synthesis of Substrates}

\section{General Procedure 1 (GP1)}

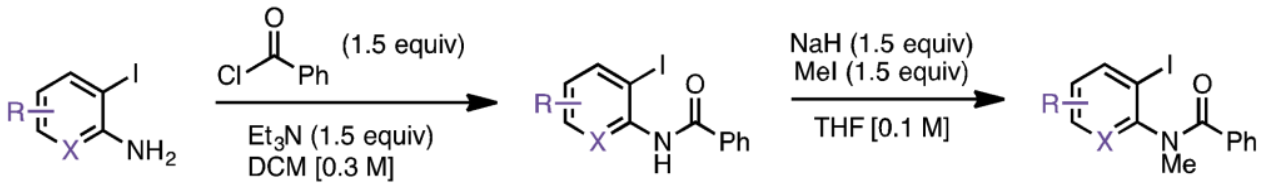

2-Iodoaniline or a derivative (typically 2 to $5 \mathrm{mmol}$ ) was stirred in DCM $[0.3 \mathrm{M}]$ with triethylamine (1.5 equiv) at $0{ }^{\circ} \mathrm{C}$. Benzoyl chloride (1.5 equiv) was added dropwise and the reaction was stirred at room temperature overnight. The reaction was quenched with $1 \mathrm{M} \mathrm{HCl}$, extracted twice in DCM. $2 \mathrm{M}$ aqueous $\mathrm{NaOH}$ was added to the organic layer and extracted twice more in DCM. The organic layer was washed with brine, dried over magnesium sulfate, filtered and concentrated under reduced pressure. The crude mixture was purified by flash chromatography $5 \%$ EtOAc/pentanes.

The resulting $\mathrm{N}$-(2-iodophenyl)benzamide was stirred in THF $[0.1 \mathrm{M}]$ at $0{ }^{\circ} \mathrm{C}$. Sodium hydride, $60 \%$ dispersion in mineral oil (1.5 equiv) was added followed by iodomethane (1.5 equiv) 5 min later and the reaction was stirred at room temperature overnight. The reaction was quenched with brine and extracted three times with EtOAc. The organic layer was dried over magnesium sulfate, filtered and concentrated under reduced pressure. The crude mixture was purified by flash chromatography $15 \%$ EtOAc/pentanes.

\section{General Procedure 2 (GP2)}
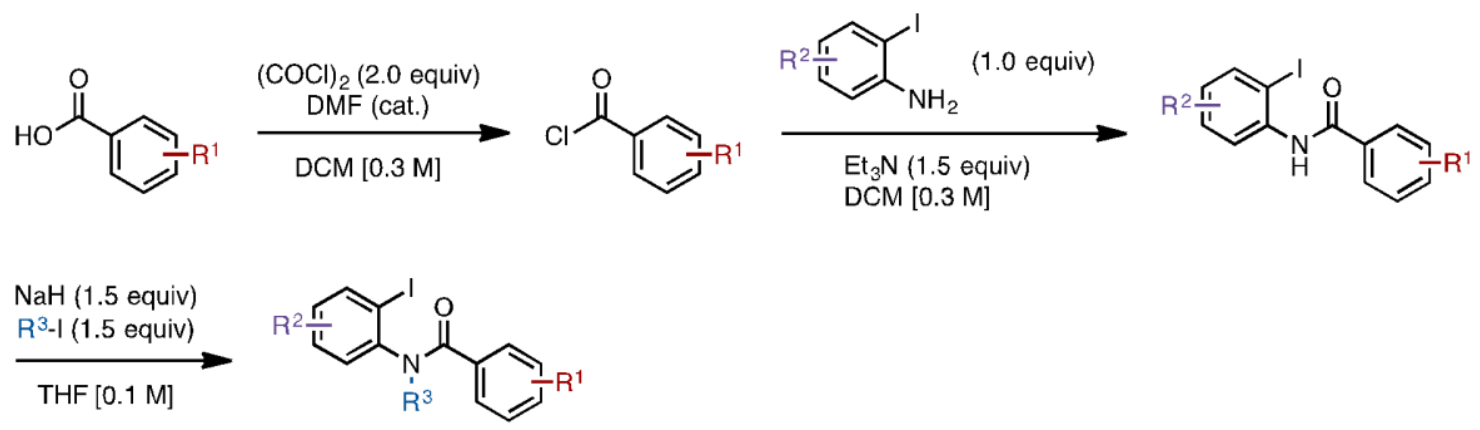

The aryl carboxylic acid (typically 2 to $5 \mathrm{mmol}$ ) and 3 drops of DMF were stirred in DCM [0.3 M] at $0{ }^{\circ} \mathrm{C}$. Oxalyl chloride ( 2.0 equiv) was added dropwise to this mixture and the reaction was stirred at room temperature for $3 \mathrm{~h}$. The resulting solution was concentration under reduced pressure to provide the acyl chloride as a yellow slurry that was used without further purification.

The crude acyl chloride was taken up with DCM $(10 \mathrm{~mL})$ and the resulting mixture was cooled at $0{ }^{\circ} \mathrm{C}$. A solution of 2 -iodoaniline (1 equiv) and triethylamine (1.5 equiv) in DCM $(5 \mathrm{~mL})$ was added dropwise to the acid chloride and the reaction was stirred at room temperature overnight. The reaction was quenched with $1 \mathrm{M} \mathrm{HCl}$ and extracted twice in DCM. $2 \mathrm{M} \mathrm{NaOH}$ was added to 
the organic layer and extracted twice more in DCM. The organic layer was washed with brine, dried over magnesium sulfate, filtered and concentrated under reduced pressure. The crude mixture was purified by flash chromatography.

The $N$-(2-iodophenyl)benzamide was stirred in THF $[0.1 \mathrm{M}]$ at $0{ }^{\circ} \mathrm{C}$. Sodium hydride, $60 \%$ dispersion in mineral oil ( 1.5 equiv) was added followed by iodoalkane ( 1.5 equiv) 5 min later and the reaction was stirred at room temperature overnight. The reaction was quenched with brine and extracted three times with EtOAc. The organic layer was dried over magnesium sulfate, filtered and concentrated under reduced pressure. The crude mixture was purified by flash chromatography. 


\section{Procedures for the Catalytic Reactions}

\section{General Procedure 3 (GP3)}

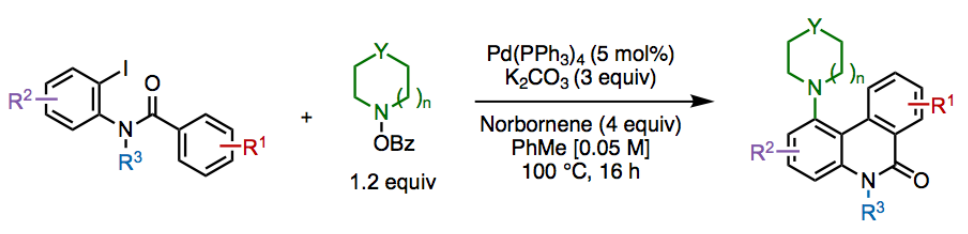

Vials and stir bars were dried in $110^{\circ} \mathrm{C}$ oven overnight prior to use. Two $2 \mathrm{dr}$ vials, one equipped with stir bar and one without were cooled to room temperature while under argon flow. Norbornene $(0.0829 \mathrm{~g}, 0.88 \mathrm{mmol}, 4.4$ equiv) was weighed in the vial without stir bar. Potassium carbonate ( $0.0829 \mathrm{~g}, 0.6 \mathrm{mmol}, 3$ equiv) starting material $(0.2 \mathrm{mmol}), O$-benzoylhydroxylamine ( 1.2 equiv) and tetrakis(triphenylphosphine)palladium(0) $(0.0116 \mathrm{~g}, 0.01 \mathrm{mmol}, 5 \mathrm{~mol} \%)$ were added in that order to the vial with stir bar. $4.4 \mathrm{~mL}$ of freshly distilled toluene were added via syringe to the norbornene-containing vial and the latter was sonicated for $15 \mathrm{~s}$ to make sure all the norbornene was fully dissolved. $4 \mathrm{~mL}$ of the resulting solution were transferred via syringe to the other vial. The vial was equipped with a Teflon-sealed cap and immediately stirred at $100{ }^{\circ} \mathrm{C}$ for $16 \mathrm{~h}$. The reaction was passed through a silica pad washing with ethyl acetate. The filtrate was concentrated under reduced pressure and the resulting residue purified by flash chromatography.

\section{General Procedure 4 (GP4)}

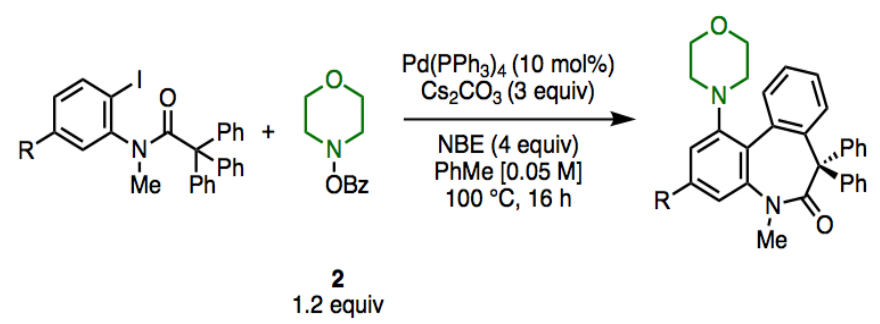

Vials and stir bars were dried in $110^{\circ} \mathrm{C}$ oven overnight prior to use. Two $2 \mathrm{dr}$ vials, one equipped with a stir bar and one without were cooled to room temperature while under argon flow. Norbornene $(0.0829 \mathrm{mg}, 0.88 \mathrm{mmol}, 4.4$ equiv) was weighed in the vial without stir bar. Cesium carbonate $(0.1955 \mathrm{mg}, 0.6 \mathrm{mmol}, 3$ equiv) starting material $(0.2 \mathrm{mmol})$, electrophilic nitrogen reagent (1.2 equiv) and tetrakis(triphenylphosphine)palladium(0) $(0.0231 \mathrm{mg}, 0.02 \mathrm{mmol}$, $10 \mathrm{~mol} \%$ ) were added in that order to the vial with stir bar. $4.4 \mathrm{~mL}$ of freshly distilled toluene were added via syringe to the norbornene-containing vial and the latter was sonicated for $15 \mathrm{~s}$ to make sure all the norbornene was fully dissolved. $4 \mathrm{~mL}$ of the resulting solution were transferred via syringe to the other vial. The vial was equipped with a Teflon-sealed cap and immediately stirred at $100{ }^{\circ} \mathrm{C}$ for $16 \mathrm{~h}$. The reaction was passed through a silica pad washing with ethyl acetate. The filtrate was concentrated under reduced pressure and the resulting residue purified by flash chromatography. 


\section{Characterization of Substrates}

$N$-(2-Iodophenyl)benzamide (1a)<smiles>O=C(Nc1ccccc1I)c1ccccc1</smiles>

Synthesized following GP1. Characterization consistent with literature. ${ }^{2}$

${ }^{1} \mathbf{H}$ NMR $\left(399 \mathrm{MHz}, \mathrm{CDCl}_{3}\right) \delta 7.79(\mathrm{~d}, J=8.0 \mathrm{~Hz}, 1 \mathrm{H}), 7.36(\mathrm{~d}, J=7.5 \mathrm{~Hz}, 2 \mathrm{H}), 7.24-7.11(\mathrm{~m}$, $4 \mathrm{H}), 7.09$ (d, $J=7.5 \mathrm{~Hz}, 1 \mathrm{H}), 6.89$ (t, $J=7.7 \mathrm{~Hz}, 1 \mathrm{H}), 3.38$ (s, 3H).

${ }^{13}$ C NMR $\left(101 \mathrm{MHz}, \mathrm{CDCl}_{3}\right) \delta 170.9,147.1,140.3,135.8,130.3,129.9,129.5,129.2,128.5$, $127.8,99.2,37.7$.

\section{$N$-Benzyl- $N$-(2-iodophenyl)benzamide (1b)}<smiles>O=C(c1ccccc1)N(Cc1ccccc1)c1ccccc1I</smiles>

Synthesized following GP2 (coupled with benzyl bromide). Characterization consistent with literature. ${ }^{3}$

${ }^{1} \mathbf{H}$ NMR $\left(399 \mathrm{MHz}, \mathrm{CDCl}_{3}\right) \delta 7.77(\mathrm{~d}, J=7.7 \mathrm{~Hz}, 1 \mathrm{H}), 7.37(\mathrm{~d}, J=7.4 \mathrm{~Hz}, 2 \mathrm{H}), 7.28(\mathrm{~m}, 5 \mathrm{H})$, $7.20(\mathrm{t}, J=7.4 \mathrm{~Hz}, 1 \mathrm{H}), 7.13(\mathrm{t}, J=7.4 \mathrm{~Hz}, 2 \mathrm{H}), 7.01(\mathrm{t}, J=7.7 \mathrm{~Hz}, 1 \mathrm{H}), 6.83(\mathrm{t}, J=7.7 \mathrm{~Hz}, 1 \mathrm{H})$, $6.62(\mathrm{~d}, J=7.7 \mathrm{~Hz}, 1 \mathrm{H}), 5.82(\mathrm{~d}, J=14.3 \mathrm{~Hz}, 1 \mathrm{H}), 4.27(\mathrm{~d}, J=14.3 \mathrm{~Hz}, 1 \mathrm{H})$.

${ }^{13} \mathrm{C}$ NMR $\left(101 \mathrm{MHz}, \mathrm{CDCl}_{3}\right) \delta 170.5,144.6,140.2,136.9,136.0,132.2,129.8,129.7,129.2$, $128.7,128.5,128.4,127.7,127.7,100.1,52.5$.

$N$-(2-Iodophenyl)- $N$-(4-methoxybenzyl)benzamide (1c)<smiles>COc1ccc(CN(C(=O)c2ccccc2)c2ccccc2I)cc1</smiles> 
The following steps are based on a literature procedure. ${ }^{5}$ 2-Iodoaniline $(5 \mathrm{mmol})$ was dissolved in ethyl acetate $[0.25 \mathrm{M}]$. $p$-Methoxybenzaldehyde (1.5 equiv) was added followed by trifluoroacetic acid ( 2 equiv). The reaction was stirred for $30 \mathrm{~min}$ then sodium triacetoxyborohydride ( 2 equiv) was added. The reduction was stirred for $2 \mathrm{~h}$ then quenched with $4 \mathrm{M} \mathrm{NaOH}$. The reaction was diluted with ethyl acetate and washed twice with brine. The organic layer was dried over magnesium sulfate, filtered, and concentrated under reduced pressure. The crude mixture was purified by flash column chromatography $5 \rightarrow 15 \%$ EtOAc/pentanes.

The alkylated amine was stirred in DCM $[0.3 \mathrm{M}]$ with triethylamine $\left(1.5\right.$ equiv) at $0{ }^{\circ} \mathrm{C}$. Benzoyl chloride (1.5 equiv) was added dropwise and the reaction was stirred at room temperature overnight. The reaction was quenched with $1 \mathrm{M} \mathrm{HCl}$, extracted twice in DCM. $2 \mathrm{M} \mathrm{NaOH}$ was added to the organic layer and extracted twice more in DCM. The organic layer was washed with brine, dried over magnesium sulfate, filtered and concentrated under reduced pressure. The crude mixture was purified by flash chromatography $5 \rightarrow 15 \%$ EtOAc/pentanes. Obtained $838 \mathrm{mg}$ of an off-white solid (70\% yield over 2 steps from 2-iodoaniline).

${ }^{1} \mathbf{H}$ NMR $\left(500 \mathrm{MHz}, \mathrm{CDCl}_{3}\right) \delta 7.77(\mathrm{~d}, J=7.8 \mathrm{~Hz}, 1 \mathrm{H}), 7.35(\mathrm{~d}, J=7.3 \mathrm{~Hz}, 2 \mathrm{H}), 7.19(\mathrm{~m}, 3 \mathrm{H})$, $7.12(\mathrm{t}, J=7.3 \mathrm{~Hz}, 2 \mathrm{H}), 7.01(\mathrm{t}, J=7.8 \mathrm{~Hz}, 1 \mathrm{H}), 6.84(\mathrm{td}, J=7.8,1.6 \mathrm{~Hz}, 1 \mathrm{H}), 6.80(\mathrm{~d}, J=8.6 \mathrm{~Hz}$, $1 \mathrm{H}), 6.59(\mathrm{dd}, J=7.8,1.6 \mathrm{~Hz}, 1 \mathrm{H}), 5.74(\mathrm{~d}, J=14.2 \mathrm{~Hz}, 1 \mathrm{H}), 4.22(\mathrm{~d}, J=14.2 \mathrm{~Hz}, 1 \mathrm{H}), 3.78$ (s, $3 \mathrm{H})$.

${ }^{13}$ C NMR $\left(126 \mathrm{MHz}, \mathrm{CDCl}_{3}\right) \delta 170.4,159.2,144.5,140.1,136.1,132.3,131.1,129.7,129.2$, $129.1,128.7,128.3,127.6,113.8,100.2,55.3,51.9$.

IR (ATR) 3037, 3012, 2964, 2838, 1629, 1566, 1510, 1470, 1435, 1393, 1243, 1176, 1024, 607

HRMS (DART) m/z: [M + H]+ Calcd for $\mathrm{C}_{21} \mathrm{H}_{19} \mathrm{INO}_{2} 444.0455$; Found 444.0450

MP $55-57{ }^{\circ} \mathrm{C}$

Ethyl $N$-benzoyl- $N$-(2-iodophenyl)glycinate (1d)<smiles>CCOC(=O)CN(C(=O)c1ccccc1)c1ccccc1I</smiles>

Synthesized following GP2 on a 2 mmol scale (coupled with iodoacetate). Purified by flash column chromatography $5 \rightarrow 15 \%$ EtOAc/pentanes. Obtained $822 \mathrm{mg}$ of an off-white solid (50\% yield over 2 steps from 2-iodoaniline). 
${ }^{1} \mathbf{H}$ NMR $\left(500 \mathrm{MHz}, \mathrm{CDCl}_{3}\right) \delta 7.82(\mathrm{dd}, J=7.9,1.5 \mathrm{~Hz}, 1 \mathrm{H}), 7.39-7.33(\mathrm{~m}, 3 \mathrm{H}), 7.23(\mathrm{t}, J=$ $7.6 \mathrm{~Hz}, 1 \mathrm{H}), 7.19-7.11(\mathrm{~m}, 2 \mathrm{H}), 6.89(\mathrm{td}, J=7.6,1.6 \mathrm{~Hz}, 1 \mathrm{H}), 5.08(\mathrm{~d}, J=17.2 \mathrm{~Hz}, 1 \mathrm{H}), 4.30-$ $4.13(\mathrm{~m}, 1 \mathrm{H}), 3.89(\mathrm{~d}, J=17.2 \mathrm{~Hz}, 1 \mathrm{H}), 1.29(\mathrm{t}, J=7.1 \mathrm{~Hz}, 3 \mathrm{H})$.

${ }^{13} \mathrm{C}$ NMR $\left(126 \mathrm{MHz}, \mathrm{CDCl}_{3}\right) \delta 170.9,168.8,145.5,139.9,134.9,132.4,130.2,129.5,129.2$, 128.3, 127.8, 99.4, 61.4, 51.3, 14.3.

IR (ATR) 3069, 2981, 1751, 1634, 1575, 1471, 1420, 1376, 1325, 1190, 1018, 996, 779, 726

HRMS (DART) m/z: [M + H]+ Calcd for $\mathrm{C}_{17} \mathrm{H}_{17} \mathrm{INO}_{3} 410.0248$; Found 410.0251

MP $71-74{ }^{\circ} \mathrm{C}$

\section{$N$-(2-Iodophenyl)- $N$,4-dimethylbenzamide (1e)}<smiles>Cc1ccc(C(=O)Nc2ccccc2I)cc1</smiles>

Synthesized following GP2. Characterization consistent with literature. ${ }^{4}$

${ }^{1} \mathbf{H}$ NMR $\left(399 \mathrm{MHz}, \mathrm{CDCl}_{3}\right) \delta 7.80(\mathrm{~d}, J=8.0 \mathrm{~Hz}, 1 \mathrm{H}), 7.25-7.16(\mathrm{~m}, 3 \mathrm{H}), 7.09(\mathrm{~d}, J=7.9 \mathrm{~Hz}$, $1 \mathrm{H}), 6.99-6.85(\mathrm{~m}, 3 \mathrm{H}), 3.36(\mathrm{~s}, 3 \mathrm{H}), 2.23(\mathrm{~s}, 3 \mathrm{H})$.

${ }^{13} \mathrm{C}$ NMR $\left(101 \mathrm{MHz}, \mathrm{CDCl}_{3}\right) \delta 171.0,147.4,140.2,140.1,132.9,130.2,129.5,129.0,128.6$, 128.4, 37.8, 21.5.

\section{$N$-(2-Iodophenyl)- $N, 3$-dimethylbenzamide (1f)}<smiles>Cc1cccc(C(=O)Nc2ccccc2I)c1</smiles>

Synthesized following GP2. Characterization consistent with literature. ${ }^{4}$

${ }^{1} \mathbf{H}$ NMR $\left(399 \mathrm{MHz}, \mathrm{CDCl}_{3}\right) \delta 7.79(\mathrm{~d}, J=8.0 \mathrm{~Hz}, 1 \mathrm{H}), 7.26(\mathrm{~s}, 1 \mathrm{H}), 7.20(\mathrm{t}, J=7.7 \mathrm{~Hz}, 1 \mathrm{H}), 7.08$ $(\mathrm{d}, J=7.7 \mathrm{~Hz}, 2 \mathrm{H}), 7.03-6.95(\mathrm{~m}, 2 \mathrm{H}), 6.88(\mathrm{t}, J=7.7 \mathrm{~Hz}, 1 \mathrm{H}), 3.36(\mathrm{~s}, 3 \mathrm{H}), 2.21(\mathrm{~s}, 3 \mathrm{H})$.

${ }^{13} \mathrm{C}$ NMR $\left(101 \mathrm{MHz}, \mathrm{CDCl}_{3}\right) \delta 171.1,147.2,140.2,137.5,130.7,130.3,129.4,129.3,129.1$, $127.5,125.4,99.2,37.7,21.3$. 


\section{$N$-(2-iodophenyl)- $N$,2-dimethylbenzamide (1g)}<smiles>Cc1ccccc1C(=O)Nc1ccccc1I</smiles>

Synthesized following GP2. Characterization consistent with literature. ${ }^{4}$ Product was isolated as a 2.3:1 mixture of rotamers. ${ }^{1} \mathrm{H} N \mathrm{NM}$ data listed is for the major rotamer. ${ }^{13} \mathrm{C} N \mathrm{~N}$ is listed for the mixture of rotamers.

${ }^{1} \mathbf{H}$ NMR $\left(500 \mathrm{MHz}, \mathrm{CDCl}_{3}\right) \delta 7.78(\mathrm{~d}, J=7.9 \mathrm{~Hz}, 1 \mathrm{H}), 7.52-7.22(\mathrm{~m}, 2 \mathrm{H}), 7.20-7.04(\mathrm{~m}, 3 \mathrm{H})$, $7.04-6.80(\mathrm{~m}, 2 \mathrm{H}), 3.41(\mathrm{~s}, 3 \mathrm{H}), 2.47$ (s, 3H).

${ }^{13} \mathrm{C}$ NMR $\left(126 \mathrm{MHz}, \mathrm{CDCl}_{3}\right) \delta 171.1,146.5,145.6,140.2,136.2,135.8,135.6,130.7,130.5$, 130.0, 129.6, 129.5, 129.3, 129.2, 129.0, 129.0, 126.5, 126.2, 126.0, 125.0, 98.9, 98.4, 39.6, 36.9, $20.3,19.5$.

\section{$N$-(2-Iodophenyl)-4-methoxy- $N$-methylbenzamide (1h)}<smiles>COc1ccc(C(=O)Nc2ccccc2I)cc1</smiles>

Synthesized following and characterization consistent with literature. ${ }^{5}$

${ }^{1} \mathbf{H}$ NMR $\left(399 \mathrm{MHz}, \mathrm{CDCl}_{3}\right) \delta 7.81(\mathrm{~d}, J=7.4 \mathrm{~Hz}, 1 \mathrm{H}), 7.31(\mathrm{~d}, J=8.3 \mathrm{~Hz}, 2 \mathrm{H}), 7.22(\mathrm{t}, J=$ $7.4 \mathrm{~Hz}, 1 \mathrm{H}), 7.09(\mathrm{~d}, J=7.4 \mathrm{~Hz}, 1 \mathrm{H}), 6.91(\mathrm{t}, J=7.4 \mathrm{~Hz}, 1 \mathrm{H}), 6.65(\mathrm{~d}, J=8.3 \mathrm{~Hz}, 2 \mathrm{H}), 3.72(\mathrm{~s}$, $3 \mathrm{H}), 3.35$ (s, 3H).

${ }^{13}$ C NMR $\left(101 \mathrm{MHz}, \mathrm{CDCl}_{3}\right) \delta 170.5,160.9,147.6,140.3,130.7,130.1,129.6,129.0,128.0$, 113.1, 99.2, 55.3, 38.0.

4-Fluoro- $N$-(2-iodophenyl)- $N$-methylbenzamide (1i)<smiles>CN(C(=O)c1ccc(F)cc1)c1ccccc1I</smiles> 
Synthesized following GP2 on a $5 \mathrm{mmol}$ scale. Purified by flash column chromatography $5 \rightarrow 15 \%$ EtOAc/pentanes. Obtained $741 \mathrm{mg}$ of an off-white solid (42\% yield over 2 steps from 2iodoaniline).

${ }^{1} \mathbf{H}$ NMR $\left(500 \mathrm{MHz}, \mathrm{CDCl}_{3}\right) \delta 7.80(\mathrm{~d}, J=7.7 \mathrm{~Hz}, 1 \mathrm{H}), 7.36(\mathrm{t}, J=8.6 \mathrm{~Hz}, 2 \mathrm{H}), 7.23(\mathrm{~d}, J=$ $7.7 \mathrm{~Hz}, 1 \mathrm{H}), 7.09(\mathrm{~d}, J=7.7 \mathrm{~Hz}, 1 \mathrm{H}), 6.92(\mathrm{t}, J=7.7 \mathrm{~Hz}, 2 \mathrm{H}), 6.83(\mathrm{t}, J=8.6 \mathrm{~Hz}, 3 \mathrm{H}), 3.36(\mathrm{~s}$, $3 \mathrm{H})$.

${ }^{13} \mathrm{C}$ NMR $\left(126 \mathrm{MHz}, \mathrm{CDCl}_{3}\right) \delta 169.8,163.4(\mathrm{~d}, J=250.6 \mathrm{~Hz}), 147.0,140.4, \delta 131.9(\mathrm{~d}, J=$ $3.5 \mathrm{~Hz}), 130.9$ (d, $J=8.6 \mathrm{~Hz}), 130.2,129.6,129.4,114.9(\mathrm{~d}, J=21.8 \mathrm{~Hz}), 99.2,37.8$.

${ }^{19}$ F NMR $\left(375 \mathrm{MHz}, \mathrm{CDCl}_{3}\right) \delta-109.87(\mathrm{td}, J=8.7,4.4 \mathrm{~Hz})$.

IR (ATR) 3055, 1628, 1602, 1509, 1468, 1368, 1237, 1158, 1020, 845, 756, 722, 600

HRMS (DART) m/z: [M + H]+ Calcd for $\mathrm{C}_{14} \mathrm{H}_{12}$ FINO 355.9942; Found 355.9946

MP $52-56{ }^{\circ} \mathrm{C}$

\section{$N$-(2-Iodophenyl)- $N$-methyl-4-(trifluoromethyl)benzamide (1j)}<smiles>CN(C(=O)c1ccc(C(F)(F)F)cc1)c1ccccc1I</smiles>

Synthesized following GP2 on a $5 \mathrm{mmol}$ scale. Purified by flash column chromatography $5 \rightarrow 15 \%$ EtOAc/pentanes. Obtained $1280 \mathrm{mg}$ of an off-white solid (63\% yield).

${ }^{1} \mathbf{H}$ NMR $\left(500 \mathrm{MHz}, \mathrm{CDCl}_{3}\right) \delta 7.80(\mathrm{dd}, J=7.8,1.4 \mathrm{~Hz}, 1 \mathrm{H}), 7.48(\mathrm{~d}, J=8.1 \mathrm{~Hz}, 2 \mathrm{H}), 7.42(\mathrm{~d}$, $J=8.1 \mathrm{~Hz}, 2 \mathrm{H}), 7.24(\mathrm{td}, J=7.8,1.4 \mathrm{~Hz}, 1 \mathrm{H}), 7.11(\mathrm{dd}, J=7.8,1.6 \mathrm{~Hz}, 1 \mathrm{H}), 6.93(\mathrm{td}, J=7.8$, $1.6 \mathrm{~Hz}, 1 \mathrm{H}), 3.39$ (s, 3H).

${ }^{13} \mathrm{C}$ NMR $\left(126 \mathrm{MHz}, \mathrm{CDCl}_{3}\right) \delta 169.4,146.3,140.4,139.3,139.3,131.56(\mathrm{q}, J=32.7 \mathrm{~Hz}), 130.1$, 129.8, 129.7, 129.7, 128.7, 127.0, 124.8 (q, $J=3.7 \mathrm{~Hz}), 123.8$ (q, $J=272.6 \mathrm{~Hz}), 99.1$, 37.6.

${ }^{19}$ F NMR $\left(375 \mathrm{MHz}, \mathrm{CDCl}_{3}\right) \delta-63.01(\mathrm{~s})$.

IR (ATR) 3055, 1737, 1631, 1470, 1369, 1306, 1157, 1106, 1020, 846, 724, 600

HRMS (DART) m/z: [M + H]+ Calcd for $\mathrm{C}_{15} \mathrm{H}_{12} \mathrm{~F}_{3} \mathrm{INO} 405.9910$; Found 405.9911

MP $61-65^{\circ} \mathrm{C}$ 


\section{$N$-(2-iodophenyl)- $N$-methyl-2-naphthamide (1k)}<smiles>CNC(=O)c1ccc2ccccc2c1</smiles>

Synthesized following GP2. Characterization consistent with literature. ${ }^{4}$

${ }^{1} \mathbf{H}$ NMR $\left(399 \mathrm{MHz}, \mathrm{CDCl}_{3}\right) \delta 7.91(\mathrm{~s}, 1 \mathrm{H}), 7.78(\mathrm{~d}, J=7.9 \mathrm{~Hz}, 1 \mathrm{H}), 7.73-7.68(\mathrm{~m}, 1 \mathrm{H}), 7.61(\mathrm{~d}$, $J=8.5 \mathrm{~Hz}, 1 \mathrm{H}), 7.49-7.40(\mathrm{~m}, 4 \mathrm{H}), 7.21-7.10(\mathrm{~m}, 2 \mathrm{H}), 6.88-6.82(\mathrm{~m}, 1 \mathrm{H}), 3.43(\mathrm{~s}, 3 \mathrm{H})$.

${ }^{13} \mathrm{C}$ NMR $\left(101 \mathrm{MHz}, \mathrm{CDCl}_{3}\right) \delta 170.9,147.2,140.3,133.8,133.2,132.3,130.3,129.5,129.2$, $129.1,128.8,127.7,127.4,127.3,126.4,125.4,99.2,37.9$.

\section{$N$-(2-Iodophenyl)- $N$-methylthiophene-2-carboxamide (11)}<smiles>CN(C(=O)c1cccs1)c1ccccc1I</smiles>

Synthesized following and consistent with literature. ${ }^{6}$

${ }^{1}$ H NMR $\left(399 \mathrm{MHz}, \mathrm{CDCl}_{3}\right) \delta 7.94(\mathrm{dd}, J=8.0,1.4 \mathrm{~Hz}, 1 \mathrm{H}), 7.42(\mathrm{td}, J=7.5,1.4 \mathrm{~Hz}, 1 \mathrm{H}), 7.34$ $(\mathrm{dd}, J=7.8,1.7 \mathrm{~Hz}, 1 \mathrm{H}), 7.30(\mathrm{~d}, J=5.0 \mathrm{~Hz}, 1 \mathrm{H}), 7.12(\mathrm{td}, J=7.7,1.7 \mathrm{~Hz}, 1 \mathrm{H}), 6.87-6.77(\mathrm{~m}$, $2 \mathrm{H}), 3.36(\mathrm{~s}, 3 \mathrm{H})$.

${ }^{13}$ C NMR (101 MHz, $\left.\mathrm{CDCl}_{3}\right) \delta 162.4, \delta 146.4,140.5,137.7,132.3,131.0,130.4,130.2,130.1$, $126.9,100.4,37.9$.

\section{$N$-(3-iodopyridin-2-yl)- $N$-methylbenzamide (1m)}<smiles>CN(C(=O)c1ccccc1)c1ncccc1I</smiles>

Synthesized following GP1 on a 2 mmol scale. Purified by flash column chromatography 15\% EtOAc/pentanes. Obtained $144 \mathrm{mg}$ of an off-white solid (19\% yield over 2 steps from 3iodopyridin-2-amine).

${ }^{1}$ H NMR $\left(500 \mathrm{MHz}, \mathrm{CDCl}_{3}\right) \delta 8.40$ (br s, 1H), 8.03 (br s, 1H), 7.40 (br s, 2H), 7.25 - 7.07 (m, $3 \mathrm{H}), 6.86$ (br s, 1H), 3.41 (s, 3H). 
${ }^{13} \mathbf{C ~ N M R}\left(126 \mathrm{MHz}, \mathrm{CDCl}_{3}\right) \delta 170.7,158.2,149.1,149.0,136.0,130.2,128.7,127.7,124.0,94.5$, 35.8 .

IR (ATR) 3106, 3033, 1739, 1651, 1558, 1413, 1352, 1132, 1067, 1014, 933, 794, 720

HRMS (DART) m/z: [M + H]+ Calcd for $\mathrm{C}_{13} \mathrm{H}_{12} \mathrm{IN}_{2} \mathrm{O}$ 338.9989; Found 338.9985

MP $135-137{ }^{\circ} \mathrm{C}$

\section{$N$-(2-iodo-5-methylphenyl)- $N$-methylbenzamide (1n)}<smiles>Cc1ccc(I)c(NC(=O)c2ccccc2)c1</smiles>

Prepared on $2 \mathrm{mmol}$ scale by GP1. Purified by flash column chromatography $15 \%$ EtOAc/pentanes. Obtained $496 \mathrm{mg}$ of an off-white solid (71\% yield over 2 steps from 2-iodo-5methylaniline).

${ }^{1}$ H NMR $\left(500 \mathrm{MHz}, \mathrm{CDCl}_{3}\right) \delta 7.62(\mathrm{~d}, J=7.9 \mathrm{~Hz}, 1 \mathrm{H}), 7.37(\mathrm{~d}, J=7.3 \mathrm{~Hz}, 2 \mathrm{H}), 7.21(\mathrm{t}, J=$ $7.3 \mathrm{~Hz}, 1 \mathrm{H}), 7.14(\mathrm{t}, J=7.3 \mathrm{~Hz}, 2 \mathrm{H}), 6.91(\mathrm{~s}, 1 \mathrm{H}), 6.70(\mathrm{~d}, J=7.9 \mathrm{~Hz}, 1 \mathrm{H}), 3.35(\mathrm{~s}, 3 \mathrm{H}), 2.17$ (s, $3 \mathrm{H})$.

${ }^{13} \mathbf{C}$ NMR $\left(126 \mathrm{MHz}, \mathrm{CDCl}_{3}\right) \delta 170.9,146.8,139.8,139.8,135.9,130.9,130.2,129.8,128.5$, 127.7, 94.9, 37.7, 20.8 .

IR (ATR) 2971, 1737, 1635, 1558, 1418, 1354, 1304, 1015, 722, 698

HRMS (DART) m/z: [M + H]+ Calcd for $\mathrm{C}_{15} \mathrm{H}_{15} \mathrm{INO} 352.0193$; Found 352.0198

MP $131-133{ }^{\circ} \mathrm{C}$

\section{N-(5-chloro-2-iodophenyl)-N-methylbenzamide (10)}<smiles>O=C(c1ccccc1)[N+]([O-])c1cc(Cl)ccc1I</smiles>

Prepared on 2 mmol scale by GP1. Purified by flash column chromatography $15 \%$ EtOAc/pentanes. Obtained $291 \mathrm{mg}$ of a white solid (39\% yield over 2 steps from 5-chloro-2iodoaniline). 
${ }^{1} \mathbf{H}$ NMR $\left(500 \mathrm{MHz}, \mathrm{CDCl}_{3}\right) \delta 7.68(\mathrm{~d}, J=8.5 \mathrm{~Hz}, 1 \mathrm{H}), 7.36(\mathrm{~d}, J=7.5 \mathrm{~Hz}, 2 \mathrm{H}), 7.25-7.05(\mathrm{~m}$, $4 \mathrm{H}), 6.90(\mathrm{~d}, J=8.5 \mathrm{~Hz}, 1 \mathrm{H}), 3.35(\mathrm{~s}, 3 \mathrm{H})$.

${ }^{13}$ C NMR $\left(126 \mathrm{MHz}, \mathrm{CDCl}_{3}\right) \delta 170.7,148.3,140.9,135.4,135.1,130.4,130.3,129.5,128.4$, 127.9, 96.6, 37.6.

IR (ATR) 3055, 2927, 1738, 1634, 1556, 1424, 1358, 1094, 1012, 722, 698

HRMS (DART) m/z: [M + H]+ Calcd for $\mathrm{C}_{14} \mathrm{H}_{12} \mathrm{ClINO} 371.9647$; Found 371.9647

MP $151-153{ }^{\circ} \mathrm{C}$

\section{$N$-(2-iodophenyl)- $N$-methyl-2,2,2-triphenylacetamide (5a)}<smiles>CN(C(=O)C(c1ccccc1)(c1ccccc1)c1ccccc1)c1ccccc1I</smiles>

A solution of triphenylacetic acid ( $2 \mathrm{mmol}$ ) in thionyl chloride (10 equiv) was stirred at $80{ }^{\circ} \mathrm{C}$ for $2.5 \mathrm{~h}$. The reaction was concentrated under reduced pressure, and the crude 2-phenylacryloyl chloride was purified by flash chromatography $5 \rightarrow 15 \%$ EtOAc/pentanes.

2-phenylacryloyl chloride was taken up with DCM $(10 \mathrm{~mL})$ and the resulting mixture was cooled at $0{ }^{\circ} \mathrm{C}$. A solution of 2-iodoaniline (1 equiv) and triethylamine (1.5 equiv) in DCM $(5 \mathrm{~mL})$ was added dropwise to the mixture and the reaction was stirred at room temperature overnight. The reaction was quenched with $1 \mathrm{M} \mathrm{HCl}$ and extracted twice in DCM. $2 \mathrm{M} \mathrm{NaOH}$ was added to the organic layer and extracted twice more in DCM. The organic layer was washed with brine, dried over magnesium sulfate, filtered and concentrated under reduced pressure. The crude mixture was purified by flash chromatography.

The resulting $N$-(2-iodophenyl)-2,2,2-triphenylacetamide was stirred in DMF $[0.1 \mathrm{M}]$ at $0{ }^{\circ} \mathrm{C}$. Sodium hydride, $60 \%$ dispersion in mineral oil (1.5 equiv) was added followed by iodomethane (1.5 equiv) 5 min later and the reaction was stirred at room temperature overnight. The reaction was quenched with brine and extracted three times with EtOAc. The organic layer was dried over magnesium sulfate, filtered and concentrated under reduced pressure. The crude mixture was purified by flash chromatography $5 \rightarrow 15 \%$ EtOAc/pentanes. Obtained $705 \mathrm{mg}$ of a white solid (35\% yield over 3 steps from triphenylacetic acid).

${ }^{1} \mathbf{H}$ NMR $\left(500 \mathrm{MHz}, \mathrm{CDCl}_{3}\right) \delta 7.90(\mathrm{~d}, J=8.9 \mathrm{~Hz}, 1 \mathrm{H}), 7.48(\mathrm{~d}, J=7.3 \mathrm{~Hz}, 6 \mathrm{H}), 7.40-7.31(\mathrm{~m}$, $7 \mathrm{H}), 7.26(\mathrm{t}, J=7.3 \mathrm{~Hz}, 3 \mathrm{H}), 7.08(\mathrm{dd}, J=7.9,1.6 \mathrm{~Hz}, 1 \mathrm{H}), 6.99(\mathrm{t}, J=7.9 \mathrm{~Hz}, 1 \mathrm{H}), 2.62(\mathrm{~s}, 3 \mathrm{H})$.

${ }^{13} \mathbf{C}$ NMR $\left(126 \mathrm{MHz}, \mathrm{CDCl}_{3}\right) \delta 172.9,148.3,142.7,140.2,130.7,129.7,129.0,128.5,127.9$, $126.8,97.5,67.9,40.1$. 
IR (ATR) 3057, 1650, 1596, 1578, 1492, 1471, 1445, 1347, 1105, 1003, 899, 743, 698

HRMS (DART) m/z: [M + H]+ Calcd for $\mathrm{C}_{27} \mathrm{H}_{23} \mathrm{INO} 504.0819$; Found 504.0813

MP $182-184{ }^{\circ} \mathrm{C}$

$N$-(2-Iodo-5-methylphenyl)- $N$-methyl-2,2,2-triphenylacetamide (5b)<smiles>Cc1ccc(I)c(N(C)C(=O)C(c2ccccc2)(c2ccccc2)c2ccccc2)c1</smiles>

A solution of triphenylacetic acid ( $2 \mathrm{mmol}$ ) in thionyl chloride (10 equiv) was stirred at $80{ }^{\circ} \mathrm{C}$ for $2.5 \mathrm{~h}$. The reaction was concentrated under reduced pressure, and the crude 2-phenylacryloyl chloride was purified by flash chromatography $5 \rightarrow 15 \%$ EtOAc/pentanes.

To a solution of 2-iodo-5-methylaniline (1.1 equiv) in dry THF at $-78{ }^{\circ} \mathrm{C}$ was added MeLi [1.6 M in $\mathrm{Et}_{2} \mathrm{O}$ ] (1.0 equiv) dropwise and the reaction mixture was stirred for $1 \mathrm{~h}$. A $1 \mathrm{M}$ solution of 2phenylacryloyl chloride in dry THF was added dropwise to the reaction mixture, the latter was warmed up to room temperature and stirred overnight. The reaction was quenched with brine and extracted three times with EtOAc. The organic layer was dried over magnesium sulfate, filtered and concentrated under reduced pressure. The crude mixture was purified by flash column chromatography $5 \rightarrow 15 \%$ EtOAc/pentanes.

The resulting $N$-(2-iodo-5-methylphenyl)-2,2,2-triphenylacetamide was stirred in DMF $[0.1 \mathrm{M}]$ at $0{ }^{\circ} \mathrm{C}$. Sodium hydride, $60 \%$ dispersion in mineral oil (1.5 equiv) was added followed by iodomethane (1.5 equiv) 5 min later and the reaction was stirred at room temperature overnight. The reaction was quenched with brine and extracted three times with EtOAc. The organic layer was dried over magnesium sulfate, filtered and concentrated under reduced pressure. The crude was purified by flash chromatography $5 \rightarrow 15 \%$ EtOAc/pentanes. Obtained $384 \mathrm{mg}$ of a white solid (19\% yield over 3 steps from triphenylacetic acid).

${ }^{1} \mathbf{H}$ NMR $\left(500 \mathrm{MHz}, \mathrm{CDCl}_{3}\right) \delta 7.75(\mathrm{~d}, J=8.1 \mathrm{~Hz}, 1 \mathrm{H}), 7.48(\mathrm{~d}, J=7.5 \mathrm{~Hz}, 6 \mathrm{H}), 7.34(\mathrm{t}, J=$ $7.5 \mathrm{~Hz}, 6 \mathrm{H}), 7.26(\mathrm{t}, J=7.5 \mathrm{~Hz}, 3 \mathrm{H}), 6.89(\mathrm{~s}, 1 \mathrm{H}), 6.82(\mathrm{~d}, J=8.1 \mathrm{~Hz}, 1 \mathrm{H}), 2.61(\mathrm{~s}, 3 \mathrm{H}), 2.30$ (s, $3 \mathrm{H})$.

${ }^{13} \mathbf{C}$ NMR $\left(126 \mathrm{MHz}, \mathrm{CDCl}_{3}\right) \delta 172.8,147.9,142.7,140.0,139.8,130.7,130.1,129.2,127.9$, $126.8,93.1,67.8,40.1,21.1$.

IR (ATR) 3059, 2924, 1643, 1597, 1492, 1443, 1336, 1106, 1018, 811, 748

HRMS (DART) m/z: [M + H]+ Calcd for $\mathrm{C}_{28} \mathrm{H}_{25} \mathrm{INO} 518.0975$; Found 518.0969 


\section{$N$-Methyl- $N$-phenyl-2-iodobenzamide (7)}<smiles>CN(C(=O)c1ccccc1I)c1ccccc1</smiles>

Synthesized following and consistent with literature. ${ }^{7}$ Product was isolated as a 2.3:1 mixture of rotamers. ${ }^{1} \mathrm{H}$ NMR data listed is for the major rotamer. ${ }^{13} \mathrm{C}$ NMR is listed for the mixture of rotamers.

${ }^{1} \mathbf{H}$ NMR $\left(399 \mathrm{MHz}, \mathrm{CDCl}_{3}\right) \delta 7.87-7.65(\mathrm{~d}, J=7.9 \mathrm{~Hz}, 1 \mathrm{H}), 7.16(\mathrm{~d}, J=7.9 \mathrm{~Hz}, 9 \mathrm{H}), 6.84(\mathrm{td}$, $J=7.6,1.8 \mathrm{~Hz}, 1 \mathrm{H}), 3.51-3.19(\mathrm{~s}, 3 \mathrm{H})$.

${ }^{13}$ C NMR $\left(101 \mathrm{MHz}, \mathrm{CDCl}_{3}\right) \delta 170.3,143.4,142.6,139.3,129.8,129.1,128.7,127.4,127.3$, 127.2, 93.8, 37.5.

\section{$N$-benzyl- $N$-(2-iodophenyl)-4-methylbenzenesulfonamide (9)}<smiles>CN(Cc1ccccc1)c1ccccc1I</smiles>

Synthesized following a reported procedure. ${ }^{8}$ The crude was purified by flash chromatography $5 \rightarrow 15 \%$ EtOAc/pentanes. Obtained $505 \mathrm{mg}$ of an off-white solid (39\% yield from $\mathrm{N}$-(2iodophenyl)-4-methylbenzenesulfonamide).

${ }^{1} \mathbf{H}$ NMR $\left(500 \mathrm{MHz}, \mathrm{CDCl}_{3}\right) \delta 7.80(\mathrm{dd}, J=7.9,1.5 \mathrm{~Hz}, 1 \mathrm{H}), 7.66(\mathrm{~d}, J=8.2 \mathrm{~Hz}, 2 \mathrm{H}), 7.29(\mathrm{~d}$, $J=8.0 \mathrm{~Hz}, 2 \mathrm{H}), 7.24-7.12(\mathrm{~m}, 6 \mathrm{H}), 6.94(\mathrm{t}, J=7.6 \mathrm{~Hz}, 1 \mathrm{H}), 6.84(\mathrm{dd}, J=8.0,1.6 \mathrm{~Hz}, 1 \mathrm{H}), 4.76$ $(\mathrm{d}, J=14.1 \mathrm{~Hz}, 1 \mathrm{H}), 4.71(\mathrm{~d}, J=14.1 \mathrm{~Hz}, 1 \mathrm{H}), 2.45(\mathrm{~s}, 3 \mathrm{H})$.

${ }^{13} \mathbf{C}$ NMR $\left(126 \mathrm{MHz}, \mathrm{CDCl}_{3}\right) \delta 143.8,141.0,140.6,136.8,135.2,131.9,129.9,129.9,129.7$, $128.6,128.4,128.3,128.1,102.4,55.4,21.7$.

IR (ATR) 3407, 3068, 3026, 2982, 2918, 2875, 1703, 1593, 1463, 1345, 1161, 1085, 1021, 872, $817,771,712,693,656$

HRMS (DART) m/z: [M + H]+ Calcd for $\mathrm{C}_{20} \mathrm{H}_{19} \mathrm{INO}_{2} \mathrm{~S}$ 464.0176; Found 464.0180

MP $120-122{ }^{\circ} \mathrm{C}$ 


\section{Unsuccessful Substrates}<smiles>CN(C(=O)c1ccco1)c1ccccc1I</smiles>

No conversion $0 \%$ yield<smiles>CN(C(=O)c1ccccc1)c1ccc(Cl)cc1I</smiles>

Full conversion $0 \%$ yield<smiles>CN(C(=O)c1cccn1C)c1ccccc1I</smiles>

No conversion $0 \%$ yield<smiles>CN(C(=O)c1ccccc1)c1ccccc1Br</smiles>

$16 \%$ conversion $13 \%$ yield<smiles>CN(C(=O)c1ccncc1)c1ccccc1I</smiles>

Full conversion $0 \%$ yield<smiles>CN(C(=O)C(C)(C)c1ccccc1)c1ccccc1I</smiles>

Full conversion $28 \%$ NMR yield, unisolable 


\section{Characterization of Products}

\section{5-Methyl-1-morpholinophenanthridin-6(5H)-one (3a)}<smiles>Cn1c(=O)c2ccccc2c2c(N3CCOCC3)cccc21</smiles>

Prepared on $2 \mathrm{mmol}$ scale by GP3. Purified by flash column chromatography 1/1/8 $\rightarrow 2 / 2 / 6$ EtOAc/ $\mathrm{CH}_{2} \mathrm{Cl}_{2} /$ pentanes. Obtained $44 \mathrm{mg}$ of an off-white solid (74\% yield).

${ }^{1} \mathbf{H}$ NMR $\left(500 \mathrm{MHz}, \mathrm{CDCl}_{3}\right) \delta 9.91(\mathrm{~d}, J=8.5 \mathrm{~Hz}, 1 \mathrm{H}), 8.54(\mathrm{dd}, J=8.5,1.6 \mathrm{~Hz}, 1 \mathrm{H}), 7.69(\mathrm{t}, J=$ $8.5 \mathrm{~Hz}, 1 \mathrm{H}), 7.53(\mathrm{t}, J=8.5, \mathrm{~Hz} 1 \mathrm{H}), 7.45(\mathrm{t}, J=8.2 \mathrm{~Hz}, 1 \mathrm{H}), 7.16(\mathrm{~d}, J=8.2 \mathrm{~Hz}, 1 \mathrm{H}), 7.04(\mathrm{~d}, J$ $=8.2 \mathrm{~Hz}, 1 \mathrm{H}), 3.99-3.92(\mathrm{~m}, 4 \mathrm{H}), 3.78(\mathrm{~s}, 3 \mathrm{H}), 3.16-3.10(\mathrm{~m}, 2 \mathrm{H}), 2.98-2.88(\mathrm{~m}, 2 \mathrm{H})$.

${ }^{13} \mathrm{C}$ NMR $\left(126 \mathrm{MHz}, \mathrm{CDCl}_{3}\right) \delta 161.8,152.1,140.4,134.1,131.6,129.5,128.5,127.5,126.0$, $125.7,113.4,112.7,110.7,67.0,52.8,30.9$.

IR (ATR) 2974, 2853, 1739, 1635, 1584, 1441, 1430, 1338, 1223, 1112, 1027, 959, 795, 721

HRMS (DART) m/z: [M + H]+ Calcd for $\mathrm{C}_{18} \mathrm{H}_{19} \mathrm{~N}_{2} \mathrm{O}_{2} 295.1441$; Found 295.1443

MP $167-172{ }^{\circ} \mathrm{C}$

\section{5-Benzyl-1-morpholinophenanthridin-6(5H)-one (3b)}<smiles>O=c1c(=O)n(Cc2ccccc2)c2cccc(N3CCOCC3)c2c2ccccc12</smiles>

Prepared on $2 \mathrm{mmol}$ scale by GP3. Purified by flash column chromatography 1/1/8 $\rightarrow 2 / 2 / 6$ EtOAc/ $\mathrm{CH}_{2} \mathrm{Cl}_{2} /$ pentanes. Obtained $63 \mathrm{mg}$ of an off-white solid (85\% yield). 
${ }^{1} \mathbf{H}$ NMR $\left(500 \mathrm{MHz}, \mathrm{CDCl}_{3}\right) \delta 9.98(\mathrm{dd}, J=8.5,1.1 \mathrm{~Hz}, 1 \mathrm{H}), 8.63(\mathrm{dd}, J=8.0,1.6 \mathrm{~Hz}, 1 \mathrm{H}), 7.75$ (ddd, $J=8.5,7.0,1.6 \mathrm{~Hz}, 1 \mathrm{H}), 7.58$ (ddd, $J=8.0,7.0,1.1 \mathrm{~Hz}, 1 \mathrm{H}), 7.33-7.18(\mathrm{~m}, 6 \mathrm{H}), 7.06$ (dd, $J=8.2,1.1 \mathrm{~Hz}, 1 \mathrm{H}), 7.00(\mathrm{dd}, J=8.2,1.1 \mathrm{~Hz}, 1 \mathrm{H}), 5.65(\mathrm{br} \mathrm{s}, 2 \mathrm{H}), 4.02-3.92(\mathrm{~m}, 4 \mathrm{H}), 3.15(\mathrm{dd}$, $J=11.6,1.9 \mathrm{~Hz}, 2 \mathrm{H}), 2.99-2.90(\mathrm{~m}, 2 \mathrm{H})$.

${ }^{13} \mathrm{C}$ NMR $\left(126 \mathrm{MHz}, \mathrm{CDCl}_{3}\right) \delta 162.0,152.1,139.7,136.9,134.4,131.9,129.4,128.9,128.8$, $127.6,127.2,126.6,126.1,125.5,113.5,113.0,111.8,67.0,52.8,47.4$.

IR (ATR) 2851, 1737, 1635, 1590, 1490, 1429, 1335, 1226, 1107, 960, 799, 724,

HRMS (DART) m/z: [M + H]+ Calcd for $\mathrm{C}_{24} \mathrm{H}_{23} \mathrm{~N}_{2} \mathrm{O}_{2}$ 371.1754; Found 371.1759

MP $136-139{ }^{\circ} \mathrm{C}$

\section{5-(4-Methoxybenzyl)-1-morpholinophenanthridin-6(5H)-one (3c)}<smiles>COc1ccc(Cn2c(=O)c3ccccc3c3c(N4CCOCC4)cccc32)cc1</smiles>

Prepared on 2 mmol scale by GP3. Purified by flash column chromatography 1/1/8 $\rightarrow 2 / 2 / 6$ EtOAc/ $\mathrm{CH}_{2} \mathrm{Cl}_{2} /$ pentanes. Obtained $53 \mathrm{mg}$ of a beige solid (67\% yield).

${ }^{1} \mathbf{H}$ NMR $\left(500 \mathrm{MHz}, \mathrm{CDCl}_{3}\right) \delta 9.97(\mathrm{dd}, J=8.5,0.7 \mathrm{~Hz}, 1 \mathrm{H}), 8.62(\mathrm{dd}, J=8.0,1.1 \mathrm{~Hz}, 1 \mathrm{H}), 7.74$ (ddd, $J=8.5,7.1,1.6 \mathrm{~Hz}, 1 \mathrm{H}), 7.57(\mathrm{ddd}, J=8.0,7.1,1.1 \mathrm{~Hz}, 1 \mathrm{H}), 7.31(\mathrm{t}, J=8.2 \mathrm{~Hz}, 1 \mathrm{H}), 7.19$ $(\mathrm{d}, J=8.8 \mathrm{~Hz}, 2 \mathrm{H}), 7.10(\mathrm{dd}, J=8.2,1.1 \mathrm{~Hz}, 1 \mathrm{H}), 6.99(\mathrm{dd}, J=8.2,1.1 \mathrm{~Hz}, 1 \mathrm{H}), 6.83(\mathrm{~d}, J=$ $8.8 \mathrm{~Hz}, 2 \mathrm{H}), 5.57$ (br s, 2H), $4.00-3.88$ (m, 4H), 3.75 (s, 3H), 3.14 (dd, J = 11.6, $1.9 \mathrm{~Hz}, 2 \mathrm{H})$, $3.00-2.86(\mathrm{~m}, 2 \mathrm{H})$.

${ }^{13} \mathrm{C}$ NMR $\left(126 \mathrm{MHz}, \mathrm{CDCl}_{3}\right) \delta 162.0,158.8,152.1,139.7,134.4,131.9,129.4,128.9,128.8$, $127.9,127.6,126.1,125.6,114.3,113.5,113.0,111.8,67.0,55.4,52.8,46.8$.

IR (ATR) 2953, 2850, 2832, 1738, 1645, 1585, 1511, 1443, 1243, 1112, 1033, 958, 797, 724

HRMS (DART) m/z: [M + H]+ Calcd for $\mathrm{C}_{25} \mathrm{H}_{25} \mathrm{~N}_{2} \mathrm{O}_{3} 401.1860$; Found 401.1861

MP $100-104{ }^{\circ} \mathrm{C}$ 
Ethyl 2-(1-morpholino-6-oxophenanthridin-5(6H)-yl)acetate (3d)<smiles>CCOC(=O)Cn1c(=O)c2ccccc2c2c(N3CCOCC3)cccc21</smiles>

Prepared on $2 \mathrm{mmol}$ scale by GP3. Purified by flash column chromatography 1/1/8 $\rightarrow 2 / 2 / 6$ EtOAc/ $\mathrm{CH}_{2} \mathrm{Cl}_{2} /$ pentanes. Obtained $60 \mathrm{mg}$ of a beige solid ( $82 \%$ yield).

${ }^{1} \mathbf{H}$ NMR $\left(500 \mathrm{MHz}, \mathrm{CDCl}_{3}\right) \delta 9.93(\mathrm{dd}, J=8.6,0.6 \mathrm{~Hz}, 1 \mathrm{H}), 8.53(\mathrm{ddd}, J=8.0,1.6,0.6 \mathrm{~Hz}, 1 \mathrm{H})$, $7.72(\mathrm{ddd}, J=8.6,7.1,1.6 \mathrm{~Hz}, 1 \mathrm{H}), 7.53(\mathrm{ddd}, J=8.0,7.1,1.1 \mathrm{~Hz}, 1 \mathrm{H}), 7.40(\mathrm{t}, J=8.2 \mathrm{~Hz}, 1 \mathrm{H})$, $7.04(\mathrm{dd}, J=8.2,1.0 \mathrm{~Hz}, 1 \mathrm{H}), 6.86(\mathrm{dd}, J=8.2,1.0 \mathrm{~Hz}, 1 \mathrm{H}), 5.17(\mathrm{~s}, 2 \mathrm{H}), 4.24(\mathrm{q}, J=7.1 \mathrm{~Hz}$, $2 \mathrm{H}), 4.00-3.88(\mathrm{~m}, 4 \mathrm{H}), 3.13(\mathrm{dd}, J=11.6,2.0 \mathrm{~Hz}, 2 \mathrm{H}), 2.99-2.86(\mathrm{~m}, 2 \mathrm{H}), 1.26(\mathrm{t}, J=7.1 \mathrm{~Hz}$, $3 \mathrm{H})$.

${ }^{13} \mathrm{C}$ NMR $\left(126 \mathrm{MHz}, \mathrm{CDCl}_{3}\right) \delta 168.6,161.7,152.4,139.5,134.4,132.1,129.6,128.7,127.6$, $126.2,125.2,113.8,112.9,110.1,67.0,61.8,52.8,45.2,14.3$.

IR (ATR) 2962, 2848, 2818, 1741, 1647, 1587, 1443, 1344, 1212, 1117, 969, 795, 722

HRMS (DART) m/z: [M + H]+ Calcd for $\mathrm{C}_{21} \mathrm{H}_{23} \mathrm{~N}_{2} \mathrm{O}_{4}$ 367.1652; Found 367.1652

MP $160-162{ }^{\circ} \mathrm{C}$

\section{5,9-Dimethyl-1-morpholinophenanthridin-6(5H)-one (3e)}<smiles>Cc1ccc2c(=O)n(C)c3cccc(N4CCOCC4)c3c2c1</smiles>

Prepared on $2 \mathrm{mmol}$ scale by GP3. Purified by flash column chromatography 1/1/8 $\rightarrow$ 2/2/6 EtOAc/ $\mathrm{CH}_{2} \mathrm{Cl}_{2} /$ pentanes. Obtained $46 \mathrm{mg}$ of a beige solid (74\% yield).

${ }^{1} \mathbf{H}$ NMR $\left(500 \mathrm{MHz}, \mathrm{CDCl}_{3}\right) \delta 9.79(\mathrm{~s}, 1 \mathrm{H}), 8.42(\mathrm{~d}, J=8.1 \mathrm{~Hz}, 1 \mathrm{H}), 7.43(\mathrm{t}, J=8.2 \mathrm{~Hz}, 1 \mathrm{H}), 7.35$ $(\mathrm{dd}, J=8.1,1.7 \mathrm{~Hz}, 1 \mathrm{H}), 7.14(\mathrm{dd}, J=8.2,1.1 \mathrm{~Hz}, 1 \mathrm{H}), 7.02(\mathrm{dd}, J=8.2,1.1 \mathrm{~Hz}, 1 \mathrm{H}), 3.99-3.89$ (m, 4H), 3.76 (s, 3H), $3.14(\mathrm{dd}, J=11.7,2.0 \mathrm{~Hz}, 2 \mathrm{H}), 2.99-2.87(\mathrm{~m}, 2 \mathrm{H}), 2.55(\mathrm{~s}, 3 \mathrm{H})$. 
${ }^{13}$ C NMR $\left(126 \mathrm{MHz}, \mathrm{CDCl}_{3}\right) \delta 161.7,152.0,141.9,140.5,134.1,129.3,128.8,128.5,126.0$, $123.5,113.3,112.7,110.8,67.1,52.8,30.8,22.3$.

IR (ATR) 2963, 2852, 1738, 1637, 1446, 1340, 1223, 1111, 1034, 798, 728

HRMS (DART) m/z: [M + H]+ Calcd for $\mathrm{C}_{19} \mathrm{H}_{21} \mathrm{~N}_{2} \mathrm{O}_{2}$ 309.1598; Found 309.1602

MP $166-170{ }^{\circ} \mathrm{C}$

\section{5,8-Dimethyl-1-morpholinophenanthridin-6(5H)-one (3f)}<smiles>Cc1ccc2c(=O)n(C)c3cccc(N4CCOCC4)c3c2c1</smiles>

Prepared on $2 \mathrm{mmol}$ scale by GP3. Purified by flash column chromatography $1 / 1 / 8 \rightarrow 2 / 2 / 6$ EtOAc/ $\mathrm{CH}_{2} \mathrm{Cl}_{2} /$ pentanes. Obtained $46 \mathrm{mg}$ of a beige solid (74\% yield). Product was isolated as a 10:1 mixture of regioisomers. ${ }^{1} \mathrm{H} N \mathrm{MR}$ and ${ }^{13} \mathrm{C} N \mathrm{NR}$ data listed are for the major rotamer.

${ }^{1} \mathbf{H}$ NMR (500 MHz, $\left.\mathrm{CDCl}_{3}\right)$ Major isomer: $\delta 9.79(\mathrm{~d}, J=8.6 \mathrm{~Hz}, 1 \mathrm{H}), 8.35(\mathrm{~s}, 1 \mathrm{H}), 7.51(\mathrm{dd}, J=$ $8.6,1.8 \mathrm{~Hz}, 1 \mathrm{H}), 7.42(\mathrm{t}, J=8.2 \mathrm{~Hz}, 1 \mathrm{H}), 7.15(\mathrm{~d}, J=8.2 \mathrm{~Hz}, 1 \mathrm{H}), 7.03(\mathrm{~d}, J=8.2 \mathrm{~Hz}, 1 \mathrm{H}), 3.98-$ $3.90(\mathrm{~m}, 4 \mathrm{H}), 3.78(\mathrm{~s}, 3 \mathrm{H}), 3.13(\mathrm{dd}, J=11.6,1.9 \mathrm{~Hz}, 2 \mathrm{H}), 2.98-2.87(\mathrm{~m}, 2 \mathrm{H}), 2.50(\mathrm{~s}, 3 \mathrm{H})$.

${ }^{13}$ C NMR (126 MHz, $\left.\mathrm{CDCl}_{3}\right)$ Major isomer: $\delta 161.8,151.8,140.0,137.6,132.9,131.6,129.0$, $128.3,126.1,125.7,113.4,113.0,110.8,67.1,52.7,30.9,21.3$.

IR (ATR) 3106, 2963, 2847, 2820, 1739, 1639, 1585, 1445, 1345, 1228, 1112, 913, 839, 803, 745

HRMS (DART) m/z: [M + H]+ Calcd for $\mathrm{C}_{19} \mathrm{H}_{21} \mathrm{~N}_{2} \mathrm{O}_{2}$ 309.1598; Found 309.1605

MP $217-222{ }^{\circ} \mathrm{C}$

\section{5,7-Dimethyl-1-morpholinophenanthridin-6(5H)-one (3g)}


<smiles>Cc1cccc2c1c(=O)n(C)c1cccc(N3CCOCC3)c21</smiles>

Prepared on 2 mmol scale by GP3. Purified by flash column chromatography 1/1/8 $\rightarrow 2 / 2 / 6$ EtOAc/ $\mathrm{CH}_{2} \mathrm{Cl}_{2} /$ pentanes. Obtained $42 \mathrm{mg}$ of a beige solid (69\% yield).

${ }^{1} \mathbf{H}$ NMR $\left(500 \mathrm{MHz}, \mathrm{CDCl}_{3}\right) \delta 9.77(\mathrm{dd}, J=8.5 \mathrm{~Hz}, 1 \mathrm{H}), 7.51(\mathrm{dd}, J=8.5,7.3 \mathrm{~Hz}, 1 \mathrm{H}), 7.40(\mathrm{t}$, $J=8.2 \mathrm{~Hz}, 1 \mathrm{H}), 7.30(\mathrm{~d}, J=7.3 \mathrm{~Hz}, 1 \mathrm{H}), 7.05(\mathrm{dd}, J=8.2,1.1 \mathrm{~Hz}, 1 \mathrm{H}), 6.94(\mathrm{dd}, J=8.2,1.1 \mathrm{~Hz}$, $1 \mathrm{H}), 4.00-3.84(\mathrm{~m}, 4 \mathrm{H}), 3.70(\mathrm{~s}, 3 \mathrm{H}), 3.14(\mathrm{dd}, J=11.6,2.0 \mathrm{~Hz}, 2 \mathrm{H}), 2.94(\mathrm{~s}, 3 \mathrm{H}), 2.91-2.84$ $(\mathrm{m}, 2 \mathrm{H})$.

${ }^{13} \mathrm{C}$ NMR $\left(126 \mathrm{MHz}, \mathrm{CDCl}_{3}\right) \delta 162.6,151.6,141.3,140.4,135.8,131.2,130.5,129.3,124.5$, $123.8,112.4,112.4,109.7,67.0,52.5,30.7,24.8$.

IR (ATR) 2851, 2814, 1737, 1641, 1586, 1481, 1224, 1111, 956, 811, 728

HRMS (DART) m/z: [M + H]+ Calcd for $\mathrm{C}_{19} \mathrm{H}_{21} \mathrm{~N}_{2} \mathrm{O}_{2} 309.1598$; Found 309.1606

MP $160-163{ }^{\circ} \mathrm{C}$

\section{9-Methoxy-5-methyl-1-morpholinophenanthridin-6(5H)-one (3h)}<smiles>COc1ccc2c(=O)n(C)c3cccc(N4CCOCC4)c3c2c1</smiles>

Prepared on $2 \mathrm{mmol}$ scale by GP3. Purified by flash column chromatography 1/1/8 $\rightarrow 2 / 2 / 6$ EtOAc/ $\mathrm{CH}_{2} \mathrm{Cl}_{2} /$ pentanes. Obtained $41 \mathrm{mg}$ of a beige solid ( $62 \%$ yield).

${ }^{1} \mathbf{H}$ NMR $\left(500 \mathrm{MHz}, \mathrm{CDCl}_{3}\right) \delta 9.52(\mathrm{~d}, J=2.6 \mathrm{~Hz}, 1 \mathrm{H}), 8.48(\mathrm{~d}, J=8.9 \mathrm{~Hz}, 1 \mathrm{H}), 7.44(\mathrm{t}, J=$ $8.2 \mathrm{~Hz}, 1 \mathrm{H}), 7.14(\mathrm{dd}, J=8.2,1.1 \mathrm{~Hz}, 1 \mathrm{H}), 7.10(\mathrm{dd}, J=8.9,2.6 \mathrm{~Hz}, 1 \mathrm{H}), 7.05$ (dd, $J=8.2,1.1$ $\mathrm{Hz}, 1 \mathrm{H}), 4.01-3.89$ (m, 7H), 3.75 (s, 3H), 3.13 (dd, J=11.6, 2.0 Hz, 2H), $2.99-2.88$ (m, 2H).

${ }^{13}$ C NMR $\left(126 \mathrm{MHz}, \mathrm{CDCl}_{3}\right) \delta 162.5,161.5,152.3,140.8,135.8,130.6,129.5,119.6,114.7$, $114.7,113.5,112.9,111.0,109.9,67.1,55.8,52.9,30.7$. 
IR (ATR) 2964, 2920, 2862, 2815, 1737, 1642, 1605, 1438, 1337, 1285, 1250, 1221, 1109, 1031, 794,728

HRMS (DART) m/z: [M + H]+ Calcd for $\mathrm{C}_{19} \mathrm{H}_{21} \mathrm{~N}_{2} \mathrm{O}_{3}$ 325.1547; Found 325.1557

MP $182-186{ }^{\circ} \mathrm{C}$

9-Fluoro-5-methyl-1-morpholinophenanthridin-6(5H)-one (3i)<smiles>Cn1c(=O)c2ccc(F)cc2c2c(N3CCOCC3)cccc21</smiles>

Prepared on $2 \mathrm{mmol}$ scale by GP3. Purified by flash column chromatography 1/1/8 $\rightarrow 2 / 2 / 6$ EtOAc/ $\mathrm{CH}_{2} \mathrm{Cl}_{2} /$ pentanes. Obtained $48 \mathrm{mg}$ of a beige solid (77\% yield).

${ }^{1} \mathbf{H}$ NMR $\left(500 \mathrm{MHz}, \mathrm{CDCl}_{3}\right) \delta 9.73(\mathrm{dd}, J=13.0,2.6 \mathrm{~Hz}, 1 \mathrm{H}), 8.53(\mathrm{dd}, J=8.9,6.5 \mathrm{~Hz}, 1 \mathrm{H}), 7.47$ (t, $J=8.2 \mathrm{~Hz}, 1 \mathrm{H}), 7.22(\mathrm{ddd}, J=8.9,7.3,2.6 \mathrm{~Hz}, 1 \mathrm{H}), 7.16(\mathrm{dd}, J=8.2,1.1 \mathrm{~Hz}, 1 \mathrm{H}), 7.06(\mathrm{dd}$, $J=8.2,1.1 \mathrm{~Hz}, 1 \mathrm{H}), 4.03-3.89(\mathrm{~m}, 4 \mathrm{H}), 3.76(\mathrm{~s}, 3 \mathrm{H}), 3.10(\mathrm{dd}, J=11.6,2.0 \mathrm{~Hz}, 2 \mathrm{H}), 3.03-2.89$ $(\mathrm{m}, 2 \mathrm{H})$.

${ }^{13}$ C NMR $\left(126 \mathrm{MHz}, \mathrm{CDCl}_{3}\right) \delta 165.1(\mathrm{~d}, J=248.7 \mathrm{~Hz}), 161.0,152.4,140.8,136.2(\mathrm{~d}, J=11.2$ $\mathrm{Hz}), 131.4(\mathrm{~d}, J=9.8 \mathrm{~Hz}), 130.1,122.3(\mathrm{~d}, J=1.9 \mathrm{~Hz}), 115.6(\mathrm{~d}, J=23.1 \mathrm{~Hz}), 113.8,112.2(\mathrm{~d}$, $J=3.0 \mathrm{~Hz}), 112.1(\mathrm{~d}, J=26.9 \mathrm{~Hz}), 111.0,66.9,53.0,30.9$.

${ }^{19}$ F NMR $\left(375 \mathrm{MHz}, \mathrm{CDCl}_{3}\right) \delta-106.85(\mathrm{dt}, J=13.7,6.8 \mathrm{~Hz})$.

IR (ATR) 2959, 2850, 1644, 1588, 1443, 1342, 1109, 879, 844, 735

HRMS (DART) m/z: [M + H]+ Calcd for $\mathrm{C}_{18} \mathrm{H}_{18} \mathrm{FN}_{2} \mathrm{O}_{2} 313.1347$; Found 313.1355

MP $204-209{ }^{\circ} \mathrm{C}$

5-Methyl-1-morpholino-9-(trifluoromethyl)phenanthridin-6(5H)-one (3j) 
<smiles>Cn1c(=O)c2ccc(C(F)(F)F)cc2c2c(N3CCOCC3)cccc21</smiles>

Prepared on 2 mmol scale by GP3. Purified by flash column chromatography 1/1/8 $\rightarrow 2 / 2 / 6$ EtOAc/ $\mathrm{CH}_{2} \mathrm{Cl}_{2} /$ pentanes. Obtained $60 \mathrm{mg}$ of a beige solid ( $83 \%$ yield).

${ }^{1} \mathbf{H}$ NMR $\left(500 \mathrm{MHz}, \mathrm{CDCl}_{3}\right) \delta 10.48-10.44(\mathrm{~m}, 1 \mathrm{H}), 8.63(\mathrm{~d}, J=8.3 \mathrm{~Hz}, 1 \mathrm{H}), 7.73(\mathrm{dd}, J=8.3$, $1.2 \mathrm{~Hz}, 1 \mathrm{H}), 7.51(\mathrm{t}, J=8.2 \mathrm{~Hz}, 1 \mathrm{H}), 7.20(\mathrm{dd}, J=8.2,1.1 \mathrm{~Hz}, 1 \mathrm{H}), 7.12(\mathrm{dd}, J=8.2,1.1 \mathrm{~Hz}, 1 \mathrm{H})$, $4.00-3.89(\mathrm{~m}, 4 \mathrm{H}), 3.80(\mathrm{~s}, 3 \mathrm{H}), 3.08(\mathrm{dd}, J=11.5,1.9 \mathrm{~Hz}, 2 \mathrm{H}), 3.04-2.92(\mathrm{~m}, 2 \mathrm{H})$.

${ }^{13}$ C NMR $\left(126 \mathrm{MHz}, \mathrm{CDCl}_{3}\right) \delta 160.9,152.5,140.6,134.3,133.3(\mathrm{q}, J=32.1 \mathrm{~Hz}), 130.4,129.4$, $127.8,127.8,124.4(\mathrm{q}, J=273.1 \mathrm{~Hz}) 123.7(\mathrm{q}, J=4.2 \mathrm{~Hz}), 123.6(\mathrm{q}, J=3.7 \mathrm{~Hz}), 123.3,114.4$, $112.2,111.2,66.8,53.0,31.1$.

${ }^{19}$ F NMR $\left(375 \mathrm{MHz}, \mathrm{CDCl}_{3}\right) \delta-62.31(\mathrm{~s})$.

IR (ATR) 2849, 2821, 1738, 1646, 1588, 1452, 1231, 1107, 1068, 801, 735

HRMS (DART) m/z: [M + H]+ Calcd for $\mathrm{C}_{19} \mathrm{H}_{18} \mathrm{~F}_{3} \mathrm{~N}_{2} \mathrm{O}_{2}$ 363.1315; Found 363.1320

MP $208-210{ }^{\circ} \mathrm{C}$

\section{5-Methyl-1-morpholinobenzo[j]phenanthridin-6(5H)-one (3k)}<smiles>Cn1c(=O)c2cc3ccccc3cc2c2c(N3CCOCC3)cccc21</smiles>

Prepared on 2 mmol scale by GP3. Co-eluted with a byproduct (22\% NMR yield).

HRMS (DART) m/z: [M + H]+ Calcd for $\mathrm{C}_{22} \mathrm{H}_{21} \mathrm{~N}_{2} \mathrm{O}_{2}$ 345.1598; Found 345.1603

\section{5-Methyl-9-morpholinothieno[2,3-c]quinolin-4(5H)-one (3I)}


<smiles>Cn1c(=O)c2sccc2c2c(N3CCOCC3)cccc21</smiles>

Prepared on 2 mmol scale by GP3. Purified by flash column chromatography 1/1/8 $\rightarrow 2 / 2 / 6$ EtOAc/ $\mathrm{CH}_{2} \mathrm{Cl}_{2} /$ pentanes. Obtained $34 \mathrm{mg}$ of a light-orange solid (56\% yield).

${ }^{1} \mathbf{H}$ NMR $\left(500 \mathrm{MHz}, \mathrm{CDCl}_{3}\right) \delta 8.82(\mathrm{~d}, J=5.3 \mathrm{~Hz}, 1 \mathrm{H}), 7.76(\mathrm{~d}, J=5.3 \mathrm{~Hz}, 1 \mathrm{H}), 7.50(\mathrm{dd}, J=8.5$, $8.0 \mathrm{~Hz}, 1 \mathrm{H}), 7.26(\mathrm{dd}, J=8.5,1.0 \mathrm{~Hz}, 1 \mathrm{H}), 7.13(\mathrm{dd}, J=8.0,1.0 \mathrm{~Hz}, 1 \mathrm{H}), 4.02-3.88(\mathrm{~m}, 4 \mathrm{H})$, $3.82(\mathrm{~s}, 3 \mathrm{H}), 3.08-2.94(\mathrm{~m}, 4 \mathrm{H})$.

${ }^{13}$ C NMR $\left(126 \mathrm{MHz}, \mathrm{CDCl}_{3}\right) \delta 158.2,151.0,140.6,140.0,132.0,131.8,129.1,127.5,114.2$, $114.0,111.7,67.2,53.4,30.4$.

IR (ATR) 2956, 2857, 2818, 1738, 1628, 1583, 1461, 1232, 1111, 1010, 798, 725

HRMS (DART) m/z: [M + H]+ Calcd for $\mathrm{C}_{16} \mathrm{H}_{17} \mathrm{~N}_{2} \mathrm{O}_{2} \mathrm{~S} 301.1005$; Found 301.1012

MP $146-150{ }^{\circ} \mathrm{C}$

\section{5-Methyl-1-morpholinobenzo[c][1,8]naphthyridin-6(5H)-one (3m)}<smiles>Cn1c(=O)c2ccccc2c2c(N3CCOCC3)ccnc21</smiles>

Prepared on 2 mmol scale by GP3. Purified by flash column chromatography 1/1/8 $\rightarrow 2 / 2 / 6$ EtOAc/ $\mathrm{CH}_{2} \mathrm{Cl}_{2} /$ pentanes. Obtained $18 \mathrm{mg}$ of a beige solid (30\% yield).

${ }^{1} \mathbf{H}$ NMR $\left(500 \mathrm{MHz}, \mathrm{CDCl}_{3}\right) \delta 9.33(\mathrm{~d}, J=8.5 \mathrm{~Hz}, 1 \mathrm{H}), 8.53(\mathrm{dd}, J=8.1,1.6 \mathrm{~Hz}, 1 \mathrm{H}), 8.37(\mathrm{~d}$, $J=5.5 \mathrm{~Hz}, 1 \mathrm{H}), 7.70(\mathrm{ddd}, J=8.5,7.1,1.6 \mathrm{~Hz}, 1 \mathrm{H}), 7.55(\mathrm{ddd}, J=8.1,7.1,1.2 \mathrm{~Hz}, 1 \mathrm{H}), 6.87(\mathrm{~d}$, $J=5.5 \mathrm{~Hz}, 1 \mathrm{H}), 3.98-3.89(\mathrm{~m}, 4 \mathrm{H}), 3.88(\mathrm{~s}, 3 \mathrm{H}), 3.26(\mathrm{dd}, J=11.8,2.0 \mathrm{~Hz}, 2 \mathrm{H}), 3.01-2.90(\mathrm{~m}$, $2 \mathrm{H})$.

${ }^{13}$ C NMR $\left(126 \mathrm{MHz}, \mathrm{CDCl}_{3}\right) \delta 162.5,158.3,150.8,148.3,132.4,131.8,128.6,127.9,125.8$, 125.5, 108.6, 106.7, 66.6, 51.8, 29.6. 
IR (ATR) 2965, 2845, 1736, 1644, 1553, 1493, 1449, 1420, 1335, 1110, 1023, 960, 826, 759, 698

HRMS (DART) m/z: [M + H]+ Calcd for $\mathrm{C}_{17} \mathrm{H}_{18} \mathrm{~N}_{3} \mathrm{O}_{2}$ 296.1394; Found 296.1392

MP $125-129^{\circ} \mathrm{C}$

\section{3,5-Dimethyl-1-morpholinophenanthridin-6(5H)-one (3n)}<smiles>Cc1cc(N2CCOCC2)c2c3ccccc3c(=O)n([Y6](=O)[O-])c2c1</smiles>

Prepared on 2 mmol scale by GP3. Purified by flash column chromatography $1 / 1 / 8 \rightarrow 2 / 2 / 6$ EtOAc/ $\mathrm{CH}_{2} \mathrm{Cl}_{2} /$ pentanes. Obtained $49 \mathrm{mg}$ of a beige solid (79\% yield).

${ }^{1} \mathbf{H}$ NMR $\left(500 \mathrm{MHz}, \mathrm{CDCl}_{3}\right) \delta 9.85(\mathrm{dd}, J=8.6,0.6 \mathrm{~Hz}, 1 \mathrm{H}), 8.52$ (ddd, $\left.J=8.0,1.6,0.6 \mathrm{~Hz}, 1 \mathrm{H}\right)$, 7.67 (ddd, $J=8.6,7.0,1.6 \mathrm{~Hz}, 1 \mathrm{H}), 7.49$ (ddd, $J=8.0,7.0,1.2 \mathrm{~Hz}, 1 \mathrm{H}), 6.97(\mathrm{~s}, 1 \mathrm{H}), 6.85$ (d, $J=$ $0.9 \mathrm{~Hz}, 1 \mathrm{H}), 3.99-3.87(\mathrm{~m}, 4 \mathrm{H}), 3.77(\mathrm{~s}, 3 \mathrm{H}), 3.11(\mathrm{dd}, J=11.6,2.0 \mathrm{~Hz}, 2 \mathrm{H}), 2.98-2.87(\mathrm{~m}$, $2 \mathrm{H}), 2.47(\mathrm{~s}, 3 \mathrm{H})$.

${ }^{13}$ C NMR $\left(126 \mathrm{MHz}, \mathrm{CDCl}_{3}\right) \delta 161.9,152.0,140.3,139.7,134.2,131.6,128.5,127.0,125.8$, $125.3,114.6,111.3,110.3,67.0,52.8,30.9,22.2$.

IR (ATR) 2957, 2887, 2850, 2828, 1641, 1602, 1496, 1443, 1343, 1113, 1038, 867, 738, 692

HRMS (DART) m/z: [M + H]+ Calcd for $\mathrm{C}_{19} \mathrm{H}_{21} \mathrm{~N}_{2} \mathrm{O}_{2}$ 309.1598; Found 309.1607

MP $185-189^{\circ} \mathrm{C}$

3-Chloro-5-methyl-1-morpholinophenanthridin-6(5H)-one (3o)<smiles>Cn1c(=O)c2ccccc2c2c(N3CCOCC3)cc(Cl)cc21</smiles> 
Prepared on $2 \mathrm{mmol}$ scale by GP3. Purified by flash column chromatography 1/1/8 $\rightarrow 2 / 2 / 6$ EtOAc/ $\mathrm{CH}_{2} \mathrm{Cl}_{2} /$ pentanes. Obtained $56 \mathrm{mg}$ of a beige solid ( $86 \%$ yield).

${ }^{1} \mathbf{H}$ NMR $\left(500 \mathrm{MHz}, \mathrm{CDCl}_{3}\right) \delta 9.74(\mathrm{~d}, J=8.6 \mathrm{~Hz}, 1 \mathrm{H}), 8.51(\mathrm{dd}, J=8.0,1.6 \mathrm{~Hz}, 1 \mathrm{H}), 7.69$ (ddd, $J=8.6,7.1,1.6 \mathrm{~Hz}, 1 \mathrm{H}), 7.53(\mathrm{ddd}, J=8.1,7.1,1.2 \mathrm{~Hz}, 1 \mathrm{H}), 7.13(\mathrm{~d}, J=2.0 \mathrm{~Hz}, 1 \mathrm{H}), 6.97(\mathrm{~d}, J=$ $2.0 \mathrm{~Hz}, 1 \mathrm{H}), 3.97-3.84(\mathrm{~m}, 4 \mathrm{H}), 3.74(\mathrm{~s}, 3 \mathrm{H}), 3.11(\mathrm{dd}, J=11.5,2.0 \mathrm{~Hz}, 2 \mathrm{H}), 2.97-2.84(\mathrm{~m}$, $2 \mathrm{H})$.

${ }^{13} \mathbf{C}$ NMR $\left(126 \mathrm{MHz}, \mathrm{CDCl}_{3}\right) \delta 161.6,152.9,141.1,135.3,133.5,131.9,128.6,127.8,125.7$, $125.5,113.9,111.1,110.7,66.8,52.7,31.0$.

IR (ATR) 2972, 2920, 2849, 1738, 1646, 1572, 1488, 1414, 1337, 1215, 1109, 1029, 966, 862, 734,695

HRMS (DART) m/z: [M + H]+ Calcd for $\mathrm{C}_{18} \mathrm{H}_{18} \mathrm{ClN}_{2} \mathrm{O}_{2}$ 329.1051; Found 329.1058

MP $170-173{ }^{\circ} \mathrm{C}$

\section{5-Methyl-1-(1,4-dioxa-8-azaspiro[4.5]decan-8-yl)phenanthridin-6(5H)-one (3p)}<smiles>Cn1c(=O)c2ccccc2c2c(N3CCC4(CC3)OCCO4)cccc21</smiles>

Prepared on $2 \mathrm{mmol}$ scale by GP3. Purified by flash column chromatography 1/1/8 $\rightarrow 2 / 2 / 6$ EtOAc/ $\mathrm{CH}_{2} \mathrm{Cl}_{2} /$ pentanes. Obtained $35 \mathrm{mg}$ of a beige solid ( $50 \%$ yield).

${ }^{1} \mathbf{H}$ NMR $\left(500 \mathrm{MHz}, \mathrm{CDCl}_{3}\right) \delta 9.89(\mathrm{~d}, J=8.6 \mathrm{~Hz}, 1 \mathrm{H}), 8.54(\mathrm{dd}, J=8.0,1.7 \mathrm{~Hz}, 1 \mathrm{H}), 7.68(\mathrm{ddd}$, $J=8.6,7.1,1.7 \mathrm{~Hz}, 1 \mathrm{H}), 7.52(\mathrm{ddd}, J=8.0,7.1,1.1 \mathrm{~Hz}, 1 \mathrm{H}), 7.41(\mathrm{t}, J=8.2 \mathrm{~Hz}, 1 \mathrm{H}), 7.13(\mathrm{dd}$, $J=8.2,1.1 \mathrm{~Hz}, 1 \mathrm{H}), 7.08(\mathrm{dd}, J=8.2,1.1 \mathrm{~Hz}, 1 \mathrm{H}), 4.02(\mathrm{dd}, J=9.4,3.9 \mathrm{~Hz}, 2 \mathrm{H}), 3.99(\mathrm{dd}, J=$ 9.4, $3.9 \mathrm{~Hz}, 2 \mathrm{H}), 3.78(\mathrm{~s}, 3 \mathrm{H}), 3.30-3.23(\mathrm{~m}, 2 \mathrm{H}), 2.95(\mathrm{td}, J=12.3,2.5 \mathrm{~Hz}, 2 \mathrm{H}), 2.16(\mathrm{td}, J=$ $13.3,4.7 \mathrm{~Hz}, 2 \mathrm{H}), 1.80(\mathrm{~d}, J=13.3 \mathrm{~Hz}, 2 \mathrm{H})$.

${ }^{13} \mathrm{C}$ NMR $\left(126 \mathrm{MHz}, \mathrm{CDCl}_{3}\right) \delta 161.8,152.5,140.2,134.4,131.7,129.2,128.4,127.4,125.9$, $125.5,114.2,112.9,110.5,107.0,64.5,50.9,35.3,30.9$.

IR (ATR) 2952, 2927, 2834, 1738, 1647, 1584, 1488, 1434, 1336, 1218, 1103, 1055, 945, 914, 796,722

HRMS (DART) m/z: [M + H]+ Calcd for $\mathrm{C}_{21} \mathrm{H}_{23} \mathrm{~N}_{2} \mathrm{O}_{3}$ 351.1703; Found 351.1711 
MP $183-185^{\circ} \mathrm{C}$

tert-Butyl 4-(5-methyl-6-oxo-5,6-dihydrophenanthridin-1-yl)piperazine-1-carboxylate (3q)<smiles>Cn1c(=O)c2ccccc2c2c(N3CCN(C(=O)OC(C)(C)C)CC3)cccc21</smiles>

Prepared on 2 mmol scale by GP3. Purified by flash column chromatography 1/1/8 $\rightarrow 2 / 2 / 6$ EtOAc/ $\mathrm{CH}_{2} \mathrm{Cl}_{2} /$ pentanes. Obtained $53 \mathrm{mg}$ of beige solid (67\% yield).

${ }^{1} \mathbf{H}$ NMR $\left(500 \mathrm{MHz}, \mathrm{CDCl}_{3}\right) \delta 9.90(\mathrm{~d}, J=8.5 \mathrm{~Hz}, 1 \mathrm{H}), 8.54(\mathrm{~d}, J=8.5 \mathrm{~Hz}, 1 \mathrm{H}), 7.67(\mathrm{t}, J=$ $8.5 \mathrm{~Hz}, 1 \mathrm{H}), 7.53(\mathrm{t}, J=8.5 \mathrm{~Hz}, 1 \mathrm{H}), 7.43(\mathrm{t}, J=8.2 \mathrm{~Hz}, 1 \mathrm{H}), 7.16(\mathrm{~d}, J=8.2 \mathrm{~Hz}, 1 \mathrm{H}), 7.02(\mathrm{~d}$, $J=8.2 \mathrm{~Hz}, 1 \mathrm{H}), 4.09(\mathrm{~s}, 2 \mathrm{H}), 3.78(\mathrm{~s}, 3 \mathrm{H}), 3.39-3.11(\mathrm{~m}, 4 \mathrm{H}), 2.84-2.65(\mathrm{~m}, 1 \mathrm{H}), 1.48(\mathrm{~s}, 9 \mathrm{H})$.

${ }^{13} \mathrm{C}$ NMR $\left(126 \mathrm{MHz}, \mathrm{CDCl}_{3}\right) \delta 161.7,155.0,152.1,140.3,134.1,131.6,129.4,128.5,127.6$, $126.1,125.8,113.8,112.9,110.9,80.1,52.4,30.9,29.8,28.5$.

IR (ATR) 2970, 2921, 2854, 1740, 1698, 1656, 1587, 1398, 1366, 1334, 1220, 1117, 798, 730

HRMS (DART) m/z: [M + H]+ Calcd for $\mathrm{C}_{23} \mathrm{H}_{28} \mathrm{~N}_{3} \mathrm{O}_{3} 394.2125$; Found 394.2130

MP $171-173{ }^{\circ} \mathrm{C}$

1-(3,4-Dihydroisoquinolin-2(1H)-yl)-5-methylphenanthridin-6(5H)-one (3r)<smiles>Cn1c(=O)c2ccccc2c2c(N3CCc4ccccc4C3)cccc21</smiles>

Prepared on 2 mmol scale by GP3. Co-eluted with a byproduct (10\% NMR yield).

HRMS (DART) m/z: [M + H] + Calcd for $\mathrm{C}_{23} \mathrm{H}_{21} \mathrm{~N}_{2} \mathrm{O} 341.1654$; Found 341.1648 


\section{1-(Azepan-1-yl)-5-methylphenanthridin-6(5H)-one (3s)}<smiles>Cn1c(=O)c2ccccc2c2c(N3CCCCCC3)cccc21</smiles>

Prepared on 2 mmol scale by GP3. Co-eluted with a byproduct (12\% NMR yield).

${ }^{1}$ H NMR $\left(500 \mathrm{MHz}, \mathrm{CDCl}_{3}\right) \delta 9.61(\mathrm{~d}, J=8.6 \mathrm{~Hz}, 1 \mathrm{H}), 8.52(\mathrm{dd}, J=7.9,1.6 \mathrm{~Hz}, 1 \mathrm{H}), 7.67$ (ddd, $J=8.6,7.0,1.6 \mathrm{~Hz}, 1 \mathrm{H}), 7.54-7.47(\mathrm{~m}, 1 \mathrm{H}), 7.39(\mathrm{t}, J=8.2 \mathrm{~Hz}, 1 \mathrm{H}), 7.11(\mathrm{dd}, J=8.2,1.1 \mathrm{~Hz}$, $1 \mathrm{H}), 7.05(\mathrm{~d}, J=8.2 \mathrm{~Hz}, 1 \mathrm{H}), 3.78(\mathrm{~s}, 2 \mathrm{H}), 3.44-3.36(\mathrm{~m}, 2 \mathrm{H}), 3.20-3.11(\mathrm{~m}, 2 \mathrm{H}), 1.84-1.65$ $(\mathrm{m}, 8 \mathrm{H})$.

${ }^{13}$ C NMR $\left(126 \mathrm{MHz}, \mathrm{CDCl}_{3}\right) \delta 161.97,154.31,140.21,134.46,131.63,129.03,128.15,127.09$, $126.26,125.42,116.48,112.89,109.24,55.89,30.85,28.44,28.30$.

HRMS (DART) m/z: [M + H]+ Calcd for $\mathrm{C}_{20} \mathrm{H}_{23} \mathrm{~N}_{2} \mathrm{O} 307.1805$; Found 307.1804

\section{5-Methyl-1-(piperidin-1-yl)phenanthridin-6(5H)-one (3t)}<smiles>Cn1c(=O)c2ccccc2c2c(N3CCCCC3)cccc21</smiles>

Prepared on $2 \mathrm{mmol}$ scale by GP3. Purified by flash column chromatography 1/1/8 $\rightarrow 2 / 2 / 6$ EtOAc/ $\mathrm{CH}_{2} \mathrm{Cl}_{2} /$ pentanes. Obtained $17 \mathrm{mg}$ of an off-white solid (29\% yield).

${ }^{1} \mathbf{H}$ NMR $\left(500 \mathrm{MHz}, \mathrm{CDCl}_{3}\right) \delta 9.94(\mathrm{~d}, J=8.6 \mathrm{~Hz}, 1 \mathrm{H}), 8.54(\mathrm{dd}, J=8.0,1.6 \mathrm{~Hz}, 1 \mathrm{H}), 7.69$ (ddd, $J=8.6,7.0,1.6 \mathrm{~Hz}, 1 \mathrm{H}), 7.52(\mathrm{ddd}, J=8.0,7.0,1.2 \mathrm{~Hz}, 1 \mathrm{H}), 7.42(\mathrm{t}, J=8.2 \mathrm{~Hz}, 1 \mathrm{H}), 7.11(\mathrm{dd}, J$ $=8.2,1.1 \mathrm{~Hz}, 1 \mathrm{H}), 7.05(\mathrm{dd}, J=8.2,1.1 \mathrm{~Hz}, 1 \mathrm{H}), 3.79(\mathrm{~s}, 3 \mathrm{H}), 3.28(\mathrm{~d}, J=10.1 \mathrm{~Hz}, 2 \mathrm{H}), 2.62(\mathrm{td}$, $J=11.7,2.5 \mathrm{~Hz}, 2 \mathrm{H}), 1.93-1.81(\mathrm{~m}, 3 \mathrm{H}), 1.78-1.71(\mathrm{~m}, 2 \mathrm{H}), 1.40-1.28(\mathrm{~m}, 1 \mathrm{H})$.

${ }^{13} \mathrm{C}$ NMR $\left(126 \mathrm{MHz}, \mathrm{CDCl}_{3}\right) \delta 161.9,153.8,140.2,134.6,131.5,129.2,128.2,127.3,126.4$, $125.4,113.9,113.0,110.0,54.1,30.9,26.2,24.2$. 
IR (ATR) 2930, 2855, 2811, 1737, 1639, 1585, 1484, 1430, 1337, 1223, 1105, 1019, 951, 795 , 721

HRMS (DART) m/z: $[\mathrm{M}+\mathrm{H}]+$ Calcd for $\mathrm{C}_{19} \mathrm{H}_{21} \mathrm{~N}_{2} \mathrm{O}$ 293.1648; Found 293.1660

MP $130-133{ }^{\circ} \mathrm{C}$

5-Methyl-1-(pyrrolidin-1-yl)phenanthridin-6(5H)-one (3u)<smiles>Cn1c(=O)c2ccccc2c2c(N3CCCC3)cccc21</smiles>

Prepared on $2 \mathrm{mmol}$ scale by GP3. Purified by flash column chromatography 1/1/8 $\rightarrow 2 / 2 / 6$ EtOAc/ $\mathrm{CH}_{2} \mathrm{Cl}_{2} /$ pentanes. Obtained $9 \mathrm{mg}$ of a grey viscous solid (17\% yield).

${ }^{1} \mathbf{H}$ NMR $\left(500 \mathrm{MHz}, \mathrm{CDCl}_{3}\right) 8.96(\mathrm{~d}, J=8.5 \mathrm{~Hz}, 1 \mathrm{H}), 8.49(\mathrm{ddd}, J=8.0,1.6,0.6 \mathrm{~Hz}, 1 \mathrm{H}), 7.64$ (ddd, $J=8.5,7.0,1.6 \mathrm{~Hz}, 1 \mathrm{H}), 7.47$ (ddd, $J=8.0,7.0,1.2 \mathrm{~Hz}, 1 \mathrm{H}), 7.37(\mathrm{t}, J=8.2 \mathrm{~Hz}, 1 \mathrm{H}), 6.99-$ $6.97(\mathrm{~m}, 1 \mathrm{H}), 6.97-6.96(\mathrm{~m}, 1 \mathrm{H}), 3.77(\mathrm{~s}, 3 \mathrm{H}), 3.52(\mathrm{br} \mathrm{s}, 2 \mathrm{H}), 2.74(\mathrm{br} \mathrm{s}, 2 \mathrm{H}), 2.10-1.71(\mathrm{~m}$, $4 \mathrm{H})$.

${ }^{13} \mathbf{C}$ NMR $\left(126 \mathrm{MHz}, \mathrm{CDCl}_{3}\right) \delta 162.1,149.5,140.2,134.4,131.4,129.0,127.9,126.6,125.8$, 125.3, 111.1, 111.1, 107.6, 51.5, 30.7, 24.7.

IR (ATR) 2941, 2872, 2822, 2810, 1639, 1583, 1486, 1446, 1341, 1061, 1036, 794, 720

HRMS (DART) m/z: [M + H]+ Calcd for $\mathrm{C}_{18} \mathrm{H}_{19} \mathrm{~N}_{2} \mathrm{O}$ 279.1492; Found 279.1500

MP $65-67^{\circ} \mathrm{C}$

5-Methyl-1-morpholino-7,7-diphenyl-5,7-dihydro-6H-dibenzo $[b, d]$ azepin-6-one (6a)<smiles>CN1C(=O)C(c2ccccc2)(c2ccccc2)c2ccccc2-c2c(N3CCOCC3)cccc21</smiles> 
Prepared on $2 \mathrm{mmol}$ scale by GP4. Purified by flash column chromatography $5 \rightarrow 15 \%$ EtOAc/ $\mathrm{CH}_{2} \mathrm{Cl}_{2} /$ pentanes. Obtained $51 \mathrm{mg}$ of a white solid (55\% yield).

${ }^{1} \mathbf{H}$ NMR $\left(500 \mathrm{MHz}, \mathrm{CDCl}_{3}\right) \delta 8.24(\mathrm{dd}, J=7.9,1.6 \mathrm{~Hz}, 1 \mathrm{H}), 7.39-7.23(\mathrm{~m}, 7 \mathrm{H}), 7.07-6.96(\mathrm{~m}$, $4 \mathrm{H}), 6.93-6.86(\mathrm{~m}, 2 \mathrm{H}), 6.72(\mathrm{~d}, J=7.9 \mathrm{~Hz}, 1 \mathrm{H}), 6.67(\mathrm{dd}, J=8.2,1.1 \mathrm{~Hz}, 1 \mathrm{H}), 6.51(\mathrm{dd}, J=$ 8.2, 1.1 Hz, 1H), $3.64-3.54(\mathrm{~m}, 4 \mathrm{H}), 3.41(\mathrm{~s}, 3 \mathrm{H}), 2.70-2.64(\mathrm{~m}, 2 \mathrm{H}), 2.57-2.51(\mathrm{~m}, 2 \mathrm{H})$.

${ }^{13} \mathrm{C}$ NMR $\left(126 \mathrm{MHz}, \mathrm{CDCl}_{3}\right) \delta 173.6,149.0,142.8,142.8,141.7,139.4,134.1,131.5,130.0$, $128.8,128.6,128.4,128.1,127.6,127.4,127.4,126.9,126.8,126.5,126.1,116.5,115.0,67.3$, $66.8,51.0,39.1$.

IR (ATR) 2852, 2818, 1663, 1583, 1442, 1264, 1228, 1114, 988, 803, 741, 705

HRMS (DART) m/z: [M + H]+ Calcd for $\mathrm{C}_{31} \mathrm{H}_{29} \mathrm{~N}_{2} \mathrm{O}_{2}$ 461.2224; Found 461.2227

MP $236-240{ }^{\circ} \mathrm{C}$

\section{3,5-Dimethyl-1-morpholino-7,7-diphenyl-5,7-dihydro-6H-dibenzo[b,d]azepin-6-one (6b)}<smiles>Cc1cc(N2CCOCC2)c2c(c1)N(C)C(=O)C(c1ccccc1)(c1ccccc1)c1ccccc1-2</smiles>

Prepared on 2 mmol scale by GP4. Purified by flash column chromatography $5 \rightarrow 15 \%$ EtOAc/ $\mathrm{CH}_{2} \mathrm{Cl}_{2}$ /pentanes. Obtained $53 \mathrm{mg}$ of a white solid ( $55 \%$ yield).

${ }^{1} \mathbf{H}$ NMR $\left(500 \mathrm{MHz}, \mathrm{CDCl}_{3}\right) \delta 8.21(\mathrm{dd}, J=7.8,1.5 \mathrm{~Hz}, 1 \mathrm{H}), 7.50-7.18(\mathrm{~m}, 8 \mathrm{H}), 7.01-6.96(\mathrm{~m}$, $2 \mathrm{H}), 6.96-6.82(\mathrm{~m}, 2 \mathrm{H}), 6.70(\mathrm{~d}, J=7.8 \mathrm{~Hz}, 1 \mathrm{H}), 6.49-6.46(\mathrm{~m}, 1 \mathrm{H}), 6.32-6.28(\mathrm{~m}, 1 \mathrm{H}), 3.63-$ $3.53(\mathrm{~m}, 4 \mathrm{H}), 3.39(\mathrm{~s}, 3 \mathrm{H}), 2.68-2.61(\mathrm{~m}, 2 \mathrm{H}), 2.60-2.47(\mathrm{~m}, 2 \mathrm{H}), 2.19(\mathrm{~s}, 3 \mathrm{H})$.

${ }^{13} \mathrm{C}$ NMR $\left(126 \mathrm{MHz}, \mathrm{CDCl}_{3}\right) \delta 173.5,148.8,142.7,142.7,141.8,139.5,138.7,134.3,131.6$, $130.6,130.5,129.9,128.6,128.5,127.9,127.4,127.1,126.8,126.8,126.5,126.1,124.9,116.9$, $116.0,67.3,66.9,51.1,39.1,21.5$.

IR (ATR) 2949, 2811, 1658, 1606, 1575, 1489, 1443, 1327, 1267, 1231, 1116, 840, 749, 703

HRMS (DART) m/z: [M + H]+ Calcd for $\mathrm{C}_{32} \mathrm{H}_{31} \mathrm{~N}_{2} \mathrm{O}_{2}$ 475.2380; Found 475.2384

MP $269-273{ }^{\circ} \mathrm{C}$ 


\section{5-methyl-10-morpholinophenanthridin-6(5H)-one (8)}<smiles>Cn1c(=O)c2cccc(N3CCOCC3)c2c2ccccc21</smiles>

Synthesized following GP3 on a 0.2 mmol scale. 28\% NMR Yield.

${ }^{1} \mathbf{H}$ NMR $\left(500 \mathrm{MHz}, \mathrm{CDCl}_{3}\right) \delta 9.96(\mathrm{dd}, J=8.4,1.5 \mathrm{~Hz}, 1 \mathrm{H}), 8.34(\mathrm{dd}, J=7.8,1.5 \mathrm{~Hz}, 1 \mathrm{H}), 7.54$ $-7.48(\mathrm{~m}, 2 \mathrm{H}), 7.46(\mathrm{dd}, J=7.8,1.5 \mathrm{~Hz}, 1 \mathrm{H}), 7.40(\mathrm{dd}, J=8.4,1.3 \mathrm{~Hz}, 1 \mathrm{H}), 7.28$ (ddd, $J=8.4$, 7.1, $1.3 \mathrm{~Hz}, 1 \mathrm{H}), 4.01-3.91(\mathrm{~m}, 4 \mathrm{H}), 3.79$ (s, 3H), 3.17 (dd, $J=11.6,2.0 \mathrm{~Hz}, 2 \mathrm{H}), 3.00-2.89$ $(\mathrm{m}, 2 \mathrm{H})$.

${ }^{13} \mathrm{C}$ NMR $\left(126 \mathrm{MHz}, \mathrm{CDCl}_{3}\right) \delta 162.0,150.3,137.5,129.2,128.5,128.4,127.2,126.6,124.2$, $122.9,121.9,120.1,114.7,67.1,52.6,30.5$.

HRMS (DART) m/z: [M + H]+ Calcd for $\mathrm{C}_{18} \mathrm{H}_{19} \mathrm{~N}_{2} \mathrm{O}_{2} 295.1441$; Found 295.1439

\section{4-(5-Tosyl-5,6-dihydrophenanthridin-1-yl)morpholine (10)}<smiles>[13CH3]N1Cc2ccccc2-c2ccccc21</smiles>

Synthesized following GP3 on a $0.2 \mathrm{mmol}$ scale. Purified by flash column chromatography $5 \rightarrow 15 \%$ EtOAc/pentanes. Obtained $35 \mathrm{mg}$ of an off-white solid (42\% yield). Spectroscopic data is consistent with literature. ${ }^{9}$

${ }^{1} \mathbf{H}$ NMR $\left(500 \mathrm{MHz}, \mathrm{CDCl}_{3}\right) \delta 8.90(\mathrm{~s}, 1 \mathrm{H}), 7.81(\mathrm{~d}, J=8.1 \mathrm{~Hz}, 1 \mathrm{H}), 7.28(\mathrm{dd}, J=8.0,0.9 \mathrm{~Hz}$, $1 \mathrm{H}), 7.23(\mathrm{t}, J=8.2 \mathrm{~Hz}, 1 \mathrm{H}), 7.20-7.10(\mathrm{~m}, 5 \mathrm{H}), 6.87(\mathrm{dd}, J=8.1,0.9 \mathrm{~Hz}, 2 \mathrm{H}), 4.98(\mathrm{~s}, 2 \mathrm{H})$, $3.99-3.57(\mathrm{~m}, 4 \mathrm{H}), 3.22-2.80(\mathrm{~m}, 4 \mathrm{H}), 2.46(\mathrm{~s}, 3 \mathrm{H})$.

${ }^{13} \mathrm{C}$ NMR $\left(126 \mathrm{MHz}, \mathrm{CDCl}_{3}\right) \delta 151.4,141.4,140.6,136.2,133.0,133.0,130.3,128.6,128.6$, $127.8,127.5,127.3,122.1,118.0,115.6,114.5,66.9,52.9,52.0,22.0$. 


\section{Procedure for the $1 \mathrm{mmol}-S c a l e$ Synthesis of $\mathbf{3 j}$}

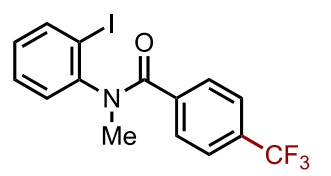

$1 \mathrm{mmol}$

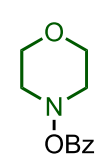

2

1.2 equiv
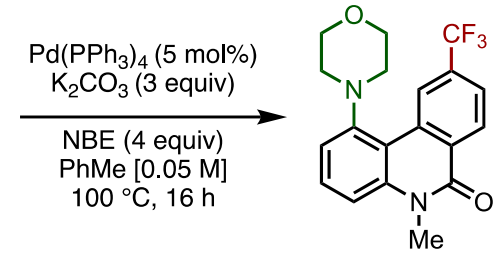

3j

Vials and stir bars were dried in $110{ }^{\circ} \mathrm{C}$ oven overnight prior to use. Two $9.5 \mathrm{dr}$ vials, one equipped with stir bar and one without were cooled to room temperature while under argon flow. Norbornene (414 mg, $4 \mathrm{mmol}, 4.4$ equiv) was weighed in the vial without stir bar. Potassium carbonate (415 mg, 3 mmol, 3 equiv), $N$-(2-iodophenyl)- $N$-methyl-4-(trifluoromethyl)benzamide (405 mg, $1 \mathrm{mmol}), \quad O$-benzoylhydroxylmorpholine $\quad(0.232 \mathrm{~g}, \quad 1.2 \mathrm{mmol}, \quad 1.2 \mathrm{equiv}) \quad$ and tetrakis(triphenylphosphine)palladium(0) $(58 \mathrm{mg}, 0.05 \mathrm{mmol}, 5 \mathrm{~mol} \%)$ were added in that order to the vial with stir bar. $22 \mathrm{~mL}$ of freshly distilled toluene were added via syringe to the norbornene-containing vial and the latter was sonicated for $15 \mathrm{~s}$ to make sure all the norbornene was fully dissolved. $20 \mathrm{~mL}$ of the resulting solution were transferred via syringe to the other vial. The vial was equipped with a Teflon-sealed cap and immediately stirred at $100{ }^{\circ} \mathrm{C}$ for $16 \mathrm{~h}$. The reaction was passed through a silica pad washing with ethyl acetate. The filtrate was concentrated under reduced pressure and the resulting residue purified by flash chromatography 1/1/8 $\rightarrow$ 2/2/6 EtOAc/ $\mathrm{CH}_{2} \mathrm{Cl}_{2} /$ pentanes. Obtained $205 \mathrm{mg}$ of a beige solid (63\% yield). 


\section{Product Derivatization}

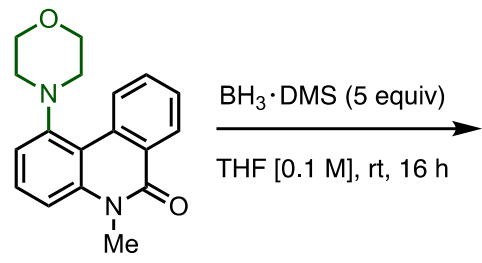

$3 a, 78 \%$ conv

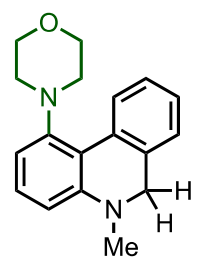

$4,69 \%$

Product 3a $(58.8 \mathrm{mg}, 0.2 \mathrm{mmol})$ was dissolved in anhydrous THF $(2 \mathrm{~mL})$. Borane-dimethylsulfide complex $(0.095 \mathrm{~mL}, 1.0 \mathrm{mmol}, 5$ equiv) was added dropwise. The reaction was stirred for $16 \mathrm{~h}$ at room temperature before being quenched with dropwise addition of $\mathrm{MeOH}$. The reaction was purified via flash column chromatography $5 \% \rightarrow 10 \% \mathrm{EtOAc/pentanes.} \mathrm{The} \mathrm{product} \mathrm{appeared} \mathrm{as}$ a white solid, $38.6 \mathrm{mg}(69 \%)$.

${ }^{1} \mathbf{H}$ NMR $\left(500 \mathrm{MHz}, \mathrm{CDCl}_{3}\right) \delta 8.99(\mathrm{~d}, J=8.0 \mathrm{~Hz}, 1 \mathrm{H}), 7.29(\mathrm{t}, J=8.0 \mathrm{~Hz}, 1 \mathrm{H}), 7.26-7.13(\mathrm{~m}$, $3 \mathrm{H}), 6.59(\mathrm{dd}, J=8.1,1.1 \mathrm{~Hz}, 1 \mathrm{H}), 6.54(\mathrm{dd}, J=8.1,1.1 \mathrm{~Hz}, 1 \mathrm{H}), 3.96(\mathrm{~s}, 2 \mathrm{H}), 3.89-3.74(\mathrm{~m}$, $4 \mathrm{H}), 3.25-3.11(\mathrm{~m}, 2 \mathrm{H}), 2.96(\mathrm{~s}, 3 \mathrm{H}), 2.94-2.82(\mathrm{~m}, 2 \mathrm{H})$.

${ }^{13}$ C NMR $\left(126 \mathrm{MHz}, \mathrm{CDCl}_{3}\right) \delta 150.9,150.0,133.7,132.5,129.3,129.3,127.0,126.6,125.3$, $125.2,115.5,108.5,107.9,67.1,55.5,52.2,39.4$.

IR (ATR) 2960, 2849, 2812, 2794, 1584, 1563, 1437, 1423, 1267, 1197, 1112, 1015, 943, 738, 718

HRMS (DART) m/z: [M + H]+ Calcd for $\mathrm{C}_{18} \mathrm{H}_{21} \mathrm{~N}_{2} \mathrm{O} 281.1648$; Found 281.1660

MP $110-114{ }^{\circ} \mathrm{C}$ 


\section{Single Crystal X-Ray}

The sample was prepared by dissolving 3a in a minimum amount of $\mathrm{CH}_{2} \mathrm{Cl}_{2}$, then recrystallizing from pentanes. Data were collected on a Bruker Kappa APEX-DUO diffractometer using $\mathrm{CuK} \alpha$ radiation from an Incoatec I $\mu \mathrm{S}$ source with multi-layer optics and a PHOTON II CMOS detector and were measured using a combination of $\phi$ scans and $\omega$ scans. The data were processed using APEX3 and SAINT (Bruker, 2019). Absorption corrections were carried out using SADABS (Bruker, 2019). The structures were solved with SHELXT (Sheldrick, 2015a) and refined using SHELXL-2018 (Sheldrick, 2015b) for full-matrix least-squares refinement that was based on $F^{2}$. $\mathrm{H}$ atoms were included in calculated positions and allowed to refine in riding-motion approximation with $\mathrm{U} \sim \mathrm{iso} \sim$ tied to the carrier atom.

Bruker (2007). APEX2, SAINT \& SADABS Bruker AXS Inc., Madison, Wisconsin, USA.

Sheldrick, G. M. (2015a). Acta Cryst. A71, 3-8.

Sheldrick, G. M. (2015b). Acta Cryst. C71, 3-8.

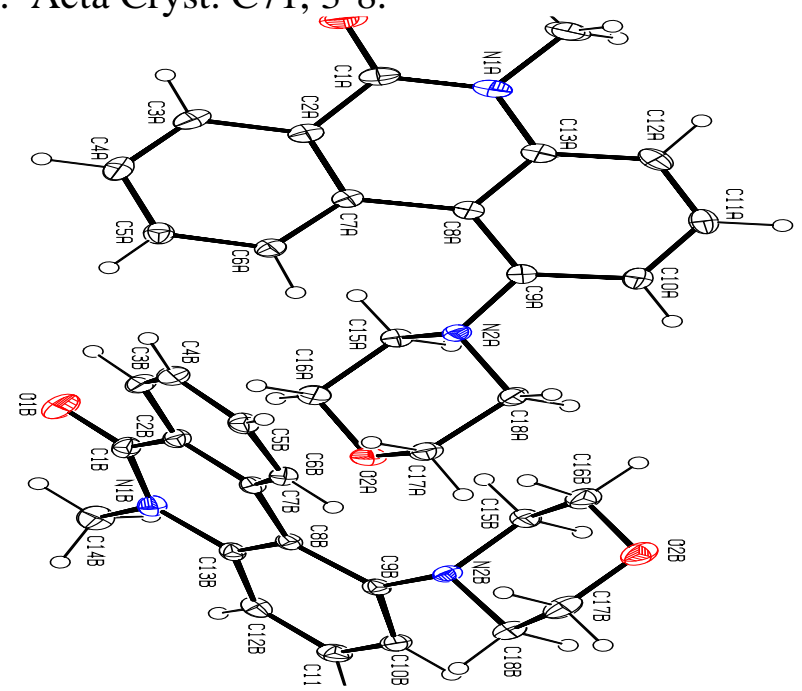

Figure S1. The asymmetric unit of compound 3 a showing the two crystallographically independent molecules. The displacement ellipsoids are drawn the $30 \%$ probability level.

Table 1. Crystal data and structure refinement for d2089_a.

Identification code

Empirical formula

Formula weight

Temperature

Wavelength

Crystal system

Space group

Unit cell dimensions d2089_a

C18 H18 N2 O2

294.34

150(2) K

$0.71073 \AA$

Triclinic

P-1

$\mathrm{a}=10.0231(8) \AA$ $\mathrm{a}=76.065(2)^{\circ}$.

$\mathrm{b}=12.1235(10) \AA$ 
Volume

$\mathrm{Z}$

Density (calculated)

Absorption coefficient

$\mathrm{F}(000)$

Crystal size

Theta range for data collection

Index ranges

Reflections collected

Independent reflections

Completeness to theta $=25.242^{\circ}$

Absorption correction

Max. and min. transmission

Refinement method

Data / restraints / parameters

Goodness-of-fit on $\mathrm{F}^{2}$

Final R indices [I $>2 \operatorname{sigma}(\mathrm{I})]$

$\mathrm{R}$ indices (all data)

Extinction coefficient

Largest diff. peak and hole $c=13.0441(10) \AA$

$\mathrm{g}=71.817(2)^{\circ}$.

$1422.5(2) \AA^{3}$

4

$1.374 \mathrm{Mg} / \mathrm{m}^{3}$

$0.091 \mathrm{~mm}^{-1}$

624

$0.370 \times 0.250 \times 0.160 \mathrm{~mm}^{3}$

1.653 to $27.550^{\circ}$.

$-13<=\mathrm{h}<=13,-15<=\mathrm{k}<=15,-16<=1<=16$

46865

$6516[\mathrm{R}(\mathrm{int})=0.0403]$

$99.7 \%$

Semi-empirical from equivalents

0.7456 and 0.7069

Full-matrix least-squares on $\mathrm{F}^{2}$

6516 / 0 / 399

1.034

$\mathrm{R} 1=0.0381, \mathrm{wR} 2=0.0925$

$\mathrm{R} 1=0.0580, \mathrm{wR} 2=0.1022$

$\mathrm{n} / \mathrm{a}$

0.232 and -0.202 e. $\AA^{-3}$ 
Table 2. Atomic coordinates ( $\mathrm{x} 10^{4}$ ) and equivalent isotropic displacement parameters $\left(\AA^{2} \times 10^{3}\right)$ for d2089_a. U(eq) is defined as one third of the trace of the orthogonalized $U^{i j}$ tensor.

\begin{tabular}{|c|c|c|c|c|}
\hline & $\mathrm{x}$ & $\mathrm{y}$ & $\mathrm{z}$ & $\mathrm{U}(\mathrm{eq})$ \\
\hline $\mathrm{O}(1 \mathrm{~B})$ & $5758(1)$ & 2182(1) & $5627(1)$ & $38(1)$ \\
\hline $\mathrm{O}(2 \mathrm{~B})$ & $13315(1)$ & $169(1)$ & $8481(1)$ & $37(1)$ \\
\hline $\mathrm{N}(1 \mathrm{~B})$ & $8123(1)$ & 1999(1) & 4781(1) & $24(1)$ \\
\hline $\mathrm{N}(2 \mathrm{~B})$ & 12007(1) & $450(1)$ & $6736(1)$ & 21(1) \\
\hline $\mathrm{C}(1 \mathrm{~B})$ & 7039(1) & 1813(1) & 5671(1) & $26(1)$ \\
\hline $\mathrm{C}(2 \mathrm{~B})$ & $7495(1)$ & $1108(1)$ & $6656(1)$ & $23(1)$ \\
\hline$C(3 B)$ & 6421(1) & 781(1) & $7529(1)$ & $29(1)$ \\
\hline $\mathrm{C}(4 \mathrm{~B})$ & $6766(1)$ & $8(1)$ & $8430(1)$ & $30(1)$ \\
\hline $\mathrm{C}(5 \mathrm{~B})$ & $8214(1)$ & $-453(1)$ & $8468(1)$ & $27(1)$ \\
\hline $\mathrm{C}(6 \mathrm{~B})$ & $9282(1)$ & $-103(1)$ & 7634(1) & $23(1)$ \\
\hline $\mathrm{C}(7 \mathrm{~B})$ & $8958(1)$ & $702(1)$ & $6702(1)$ & $20(1)$ \\
\hline $\mathrm{C}(8 \mathrm{~B})$ & $10047(1)$ & 1080(1) & $5767(1)$ & 19(1) \\
\hline $\mathrm{C}(9 \mathrm{~B})$ & $11528(1)$ & $889(1)$ & $5751(1)$ & $20(1)$ \\
\hline $\mathrm{C}(10 \mathrm{~B})$ & 12493(1) & $1166(1)$ & 4793(1) & $24(1)$ \\
\hline $\mathrm{C}(11 \mathrm{~B})$ & $12016(1)$ & $1697(1)$ & $3850(1)$ & $27(1)$ \\
\hline$C(12 B)$ & 10581(1) & $1987(1)$ & $3857(1)$ & $24(1)$ \\
\hline$C(13 B)$ & $9587(1)$ & 1681(1) & $4806(1)$ & 21(1) \\
\hline$C(14 B)$ & $7689(2)$ & $2626(1)$ & $3768(1)$ & $34(1)$ \\
\hline$C(15 B)$ & $11520(1)$ & $1334(1)$ & $7456(1)$ & $26(1)$ \\
\hline$C(16 B)$ & $11828(1)$ & $760(1)$ & $8559(1)$ & $34(1)$ \\
\hline $\mathrm{C}(17 \mathrm{~B})$ & 13774(1) & $-668(1)$ & $7767(1)$ & $34(1)$ \\
\hline $\mathrm{C}(18 \mathrm{~B})$ & $13555(1)$ & $-93(1)$ & 6642(1) & $26(1)$ \\
\hline $\mathrm{O}(1 \mathrm{~A})$ & $592(1)$ & $7048(1)$ & 9939(1) & $48(1)$ \\
\hline $\mathrm{O}(2 \mathrm{~A})$ & $8377(1)$ & $4648(1)$ & 4984(1) & $32(1)$ \\
\hline $\mathrm{N}(1 \mathrm{~A})$ & 2915(1) & $6976(1)$ & 9854(1) & $29(1)$ \\
\hline $\mathrm{N}(2 \mathrm{~A})$ & 6992(1) & $5159(1)$ & 7119(1) & 21(1) \\
\hline $\mathrm{C}(1 \mathrm{~A})$ & $1890(1)$ & $6652(1)$ & 9570(1) & $30(1)$ \\
\hline$C(2 A)$ & $2439(1)$ & $5768(1)$ & 8851(1) & $25(1)$ \\
\hline $\mathrm{C}(3 \mathrm{~A})$ & $1434(1)$ & $5258(1)$ & $8720(1)$ & $31(1)$ \\
\hline $\mathrm{C}(4 \mathrm{~A})$ & $1868(1)$ & 4349(1) & $8144(1)$ & $32(1)$ \\
\hline $\mathrm{C}(5 \mathrm{~A})$ & $3333(1)$ & 3931(1) & $7690(1)$ & $27(1)$ \\
\hline
\end{tabular}




\begin{tabular}{lllll}
$\mathrm{C}(6 \mathrm{~A})$ & $4329(1)$ & $4439(1)$ & $7798(1)$ & $23(1)$ \\
$\mathrm{C}(7 \mathrm{~A})$ & $3916(1)$ & $5383(1)$ & $8374(1)$ & $21(1)$ \\
$\mathrm{C}(8 \mathrm{~A})$ & $4934(1)$ & $5917(1)$ & $8570(1)$ & $20(1)$ \\
$\mathrm{C}(9 \mathrm{~A})$ & $6430(1)$ & $5726(1)$ & $8045(1)$ & $21(1)$ \\
$\mathrm{C}(10 \mathrm{~A})$ & $7337(1)$ & $6100(1)$ & $8438(1)$ & $25(1)$ \\
$\mathrm{C}(11 \mathrm{~A})$ & $6792(2)$ & $6740(1)$ & $9284(1)$ & $28(1)$ \\
$\mathrm{C}(12 \mathrm{~A})$ & $5339(2)$ & $7041(1)$ & $9733(1)$ & $28(1)$ \\
$\mathrm{C}(13 \mathrm{~A})$ & $4404(1)$ & $6638(1)$ & $9384(1)$ & $24(1)$ \\
$\mathrm{C}(14 \mathrm{~A})$ & $2395(2)$ & $7781(1)$ & $10647(1)$ & $44(1)$ \\
$\mathrm{C}(15 \mathrm{~A})$ & $6407(1)$ & $5823(1)$ & $6168(1)$ & $23(1)$ \\
$\mathrm{C}(16 \mathrm{~A})$ & $6846(1)$ & $5066(1)$ & $5301(1)$ & $29(1)$ \\
$\mathrm{C}(17 \mathrm{~A})$ & $8934(1)$ & $4008(1)$ & $5906(1)$ & $30(1)$ \\
$\mathrm{C}(18 \mathrm{~A})$ & $8565(1)$ & $4752(1)$ & $6776(1)$ & $26(1)$ \\
& & & & \\
\hline
\end{tabular}


Table 3. Bond lengths $[\AA ̊]$ and angles $\left[^{\circ}\right]$ for d2089_a.

\begin{tabular}{|c|c|}
\hline $\mathrm{O}(1 \mathrm{~B})-\mathrm{C}(1 \mathrm{~B})$ & $1.2340(15)$ \\
\hline $\mathrm{O}(2 \mathrm{~B})-\mathrm{C}(16 \mathrm{~B})$ & $1.4237(16)$ \\
\hline $\mathrm{O}(2 \mathrm{~B})-\mathrm{C}(17 \mathrm{~B})$ & $1.4242(18)$ \\
\hline $\mathrm{N}(1 \mathrm{~B})-\mathrm{C}(1 \mathrm{~B})$ & $1.3721(16)$ \\
\hline $\mathrm{N}(1 \mathrm{~B})-\mathrm{C}(13 \mathrm{~B})$ & $1.4040(15)$ \\
\hline $\mathrm{N}(1 \mathrm{~B})-\mathrm{C}(14 \mathrm{~B})$ & $1.4671(16)$ \\
\hline $\mathrm{N}(2 \mathrm{~B})-\mathrm{C}(9 \mathrm{~B})$ & $1.4246(15)$ \\
\hline $\mathrm{N}(2 \mathrm{~B})-\mathrm{C}(18 \mathrm{~B})$ & $1.4655(15)$ \\
\hline $\mathrm{N}(2 \mathrm{~B})-\mathrm{C}(15 \mathrm{~B})$ & $1.4716(15)$ \\
\hline $\mathrm{C}(1 \mathrm{~B})-\mathrm{C}(2 \mathrm{~B})$ & $1.4680(18)$ \\
\hline $\mathrm{C}(2 \mathrm{~B})-\mathrm{C}(3 \mathrm{~B})$ & $1.3992(17)$ \\
\hline $\mathrm{C}(2 \mathrm{~B})-\mathrm{C}(7 \mathrm{~B})$ & $1.4075(17)$ \\
\hline $\mathrm{C}(3 \mathrm{~B})-\mathrm{C}(4 \mathrm{~B})$ & $1.3707(19)$ \\
\hline $\mathrm{C}(3 \mathrm{~B})-\mathrm{H}(3 \mathrm{BA})$ & 0.9500 \\
\hline $\mathrm{C}(4 \mathrm{~B})-\mathrm{C}(5 \mathrm{~B})$ & $1.3936(19)$ \\
\hline $\mathrm{C}(4 \mathrm{~B})-\mathrm{H}(4 \mathrm{BA})$ & 0.9500 \\
\hline $\mathrm{C}(5 \mathrm{~B})-\mathrm{C}(6 \mathrm{~B})$ & $1.3791(17)$ \\
\hline $\mathrm{C}(5 \mathrm{~B})-\mathrm{H}(5 \mathrm{BA})$ & 0.9500 \\
\hline $\mathrm{C}(6 \mathrm{~B})-\mathrm{C}(7 \mathrm{~B})$ & $1.4117(17)$ \\
\hline $\mathrm{C}(6 \mathrm{~B})-\mathrm{H}(6 \mathrm{BA})$ & 0.9500 \\
\hline $\mathrm{C}(7 \mathrm{~B})-\mathrm{C}(8 \mathrm{~B})$ & $1.4732(16)$ \\
\hline $\mathrm{C}(8 \mathrm{~B})-\mathrm{C}(13 \mathrm{~B})$ & $1.4132(16)$ \\
\hline $\mathrm{C}(8 \mathrm{~B})-\mathrm{C}(9 \mathrm{~B})$ & $1.4240(16)$ \\
\hline $\mathrm{C}(9 \mathrm{~B})-\mathrm{C}(10 \mathrm{~B})$ & $1.3847(16)$ \\
\hline $\mathrm{C}(10 \mathrm{~B})-\mathrm{C}(11 \mathrm{~B})$ & $1.3859(18)$ \\
\hline $\mathrm{C}(10 \mathrm{~B})-\mathrm{H}(10 \mathrm{~A})$ & 0.9500 \\
\hline $\mathrm{C}(11 \mathrm{~B})-\mathrm{C}(12 \mathrm{~B})$ & $1.3671(18)$ \\
\hline $\mathrm{C}(11 \mathrm{~B})-\mathrm{H}(11 \mathrm{~A})$ & 0.9500 \\
\hline $\mathrm{C}(12 \mathrm{~B})-\mathrm{C}(13 \mathrm{~B})$ & $1.4014(17)$ \\
\hline $\mathrm{C}(12 \mathrm{~B})-\mathrm{H}(12 \mathrm{~A})$ & 0.9500 \\
\hline $\mathrm{C}(14 \mathrm{~B})-\mathrm{H}(14 \mathrm{~A})$ & 0.9800 \\
\hline $\mathrm{C}(14 \mathrm{~B})-\mathrm{H}(14 \mathrm{~B})$ & 0.9800 \\
\hline $\mathrm{C}(14 \mathrm{~B})-\mathrm{H}(14 \mathrm{C})$ & 0.9800 \\
\hline$C(15 B)-C(16 B)$ & $1.5120(18)$ \\
\hline
\end{tabular}




\begin{tabular}{|c|c|}
\hline $\mathrm{C}(15 \mathrm{~B})-\mathrm{H}(15 \mathrm{~A})$ & 0.9900 \\
\hline $\mathrm{C}(15 \mathrm{~B})-\mathrm{H}(15 \mathrm{~B})$ & 0.9900 \\
\hline $\mathrm{C}(16 \mathrm{~B})-\mathrm{H}(16 \mathrm{~A})$ & 0.9900 \\
\hline $\mathrm{C}(16 \mathrm{~B})-\mathrm{H}(16 \mathrm{~B})$ & 0.9900 \\
\hline$C(17 B)-C(18 B)$ & $1.5111(18)$ \\
\hline $\mathrm{C}(17 \mathrm{~B})-\mathrm{H}(17 \mathrm{~A})$ & 0.9900 \\
\hline $\mathrm{C}(17 \mathrm{~B})-\mathrm{H}(17 \mathrm{~B})$ & 0.9900 \\
\hline $\mathrm{C}(18 \mathrm{~B})-\mathrm{H}(18 \mathrm{~A})$ & 0.9900 \\
\hline $\mathrm{C}(18 \mathrm{~B})-\mathrm{H}(18 \mathrm{~B})$ & 0.9900 \\
\hline $\mathrm{O}(1 \mathrm{~A})-\mathrm{C}(1 \mathrm{~A})$ & $1.2322(16)$ \\
\hline $\mathrm{O}(2 \mathrm{~A})-\mathrm{C}(17 \mathrm{~A})$ & $1.4225(17)$ \\
\hline $\mathrm{O}(2 \mathrm{~A})-\mathrm{C}(16 \mathrm{~A})$ & $1.4260(15)$ \\
\hline $\mathrm{N}(1 \mathrm{~A})-\mathrm{C}(1 \mathrm{~A})$ & $1.3732(18)$ \\
\hline $\mathrm{N}(1 \mathrm{~A})-\mathrm{C}(13 \mathrm{~A})$ & $1.4090(16)$ \\
\hline $\mathrm{N}(1 \mathrm{~A})-\mathrm{C}(14 \mathrm{~A})$ & $1.4674(17)$ \\
\hline $\mathrm{N}(2 \mathrm{~A})-\mathrm{C}(9 \mathrm{~A})$ & $1.4211(15)$ \\
\hline $\mathrm{N}(2 \mathrm{~A})-\mathrm{C}(18 \mathrm{~A})$ & $1.4647(15)$ \\
\hline $\mathrm{N}(2 \mathrm{~A})-\mathrm{C}(15 \mathrm{~A})$ & $1.4744(15)$ \\
\hline$C(1 \mathrm{~A})-\mathrm{C}(2 \mathrm{~A})$ & $1.4661(18)$ \\
\hline $\mathrm{C}(2 \mathrm{~A})-\mathrm{C}(3 \mathrm{~A})$ & $1.4020(19)$ \\
\hline $\mathrm{C}(2 \mathrm{~A})-\mathrm{C}(7 \mathrm{~A})$ & $1.4062(17)$ \\
\hline$C(3 A)-C(4 A)$ & $1.371(2)$ \\
\hline $\mathrm{C}(3 \mathrm{~A})-\mathrm{H}(3 \mathrm{AA})$ & 0.9500 \\
\hline$C(4 A)-C(5 A)$ & $1.3939(18)$ \\
\hline $\mathrm{C}(4 \mathrm{~A})-\mathrm{H}(4 \mathrm{AA})$ & 0.9500 \\
\hline$C(5 A)-C(6 A)$ & $1.3789(17)$ \\
\hline $\mathrm{C}(5 \mathrm{~A})-\mathrm{H}(5 \mathrm{AA})$ & 0.9500 \\
\hline $\mathrm{C}(6 \mathrm{~A})-\mathrm{C}(7 \mathrm{~A})$ & $1.4093(17)$ \\
\hline $\mathrm{C}(6 \mathrm{~A})-\mathrm{H}(6 \mathrm{AA})$ & 0.9500 \\
\hline $\mathrm{C}(7 \mathrm{~A})-\mathrm{C}(8 \mathrm{~A})$ & $1.4726(17)$ \\
\hline $\mathrm{C}(8 \mathrm{~A})-\mathrm{C}(13 \mathrm{~A})$ & $1.4174(17)$ \\
\hline $\mathrm{C}(8 \mathrm{~A})-\mathrm{C}(9 \mathrm{~A})$ & $1.4287(17)$ \\
\hline$C(9 A)-C(10 A)$ & $1.3892(17)$ \\
\hline $\mathrm{C}(10 \mathrm{~A})-\mathrm{C}(11 \mathrm{~A})$ & $1.3854(18)$ \\
\hline $\mathrm{C}(10 \mathrm{~A})-\mathrm{H}(10 \mathrm{~B})$ & 0.9500 \\
\hline $\mathrm{C}(11 \mathrm{~A})-\mathrm{C}(12 \mathrm{~A})$ & $1.3708(19)$ \\
\hline
\end{tabular}




\begin{tabular}{|c|c|}
\hline $\mathrm{C}(11 \mathrm{~A})-\mathrm{H}(11 \mathrm{~B})$ & 0.9500 \\
\hline$C(12 A)-C(13 A)$ & $1.4004(19)$ \\
\hline $\mathrm{C}(12 \mathrm{~A})-\mathrm{H}(12 \mathrm{~B})$ & 0.9500 \\
\hline $\mathrm{C}(14 \mathrm{~A})-\mathrm{H}(14 \mathrm{D})$ & 0.9800 \\
\hline $\mathrm{C}(14 \mathrm{~A})-\mathrm{H}(14 \mathrm{E})$ & 0.9800 \\
\hline $\mathrm{C}(14 \mathrm{~A})-\mathrm{H}(14 \mathrm{~F})$ & 0.9800 \\
\hline $\mathrm{C}(15 \mathrm{~A})-\mathrm{C}(16 \mathrm{~A})$ & $1.5096(17)$ \\
\hline $\mathrm{C}(15 \mathrm{~A})-\mathrm{H}(15 \mathrm{C})$ & 0.9900 \\
\hline $\mathrm{C}(15 \mathrm{~A})-\mathrm{H}(15 \mathrm{D})$ & 0.9900 \\
\hline $\mathrm{C}(16 \mathrm{~A})-\mathrm{H}(16 \mathrm{C})$ & 0.9900 \\
\hline $\mathrm{C}(16 \mathrm{~A})-\mathrm{H}(16 \mathrm{D})$ & 0.9900 \\
\hline $\mathrm{C}(17 \mathrm{~A})-\mathrm{C}(18 \mathrm{~A})$ & $1.5114(18)$ \\
\hline $\mathrm{C}(17 \mathrm{~A})-\mathrm{H}(17 \mathrm{C})$ & 0.9900 \\
\hline $\mathrm{C}(17 \mathrm{~A})-\mathrm{H}(17 \mathrm{D})$ & 0.9900 \\
\hline $\mathrm{C}(18 \mathrm{~A})-\mathrm{H}(18 \mathrm{C})$ & 0.9900 \\
\hline $\mathrm{C}(18 \mathrm{~A})-\mathrm{H}(18 \mathrm{D})$ & 0.9900 \\
\hline $\mathrm{C}(16 \mathrm{~B})-\mathrm{O}(2 \mathrm{~B})-\mathrm{C}(17 \mathrm{~B})$ & $110.36(10)$ \\
\hline $\mathrm{C}(1 \mathrm{~B})-\mathrm{N}(1 \mathrm{~B})-\mathrm{C}(13 \mathrm{~B})$ & $123.84(10)$ \\
\hline $\mathrm{C}(1 \mathrm{~B})-\mathrm{N}(1 \mathrm{~B})-\mathrm{C}(14 \mathrm{~B})$ & $116.86(11)$ \\
\hline $\mathrm{C}(13 \mathrm{~B})-\mathrm{N}(1 \mathrm{~B})-\mathrm{C}(14 \mathrm{~B})$ & $119.21(10)$ \\
\hline $\mathrm{C}(9 \mathrm{~B})-\mathrm{N}(2 \mathrm{~B})-\mathrm{C}(18 \mathrm{~B})$ & $117.06(9)$ \\
\hline $\mathrm{C}(9 \mathrm{~B})-\mathrm{N}(2 \mathrm{~B})-\mathrm{C}(15 \mathrm{~B})$ & $112.55(9)$ \\
\hline $\mathrm{C}(18 \mathrm{~B})-\mathrm{N}(2 \mathrm{~B})-\mathrm{C}(15 \mathrm{~B})$ & $109.04(10)$ \\
\hline $\mathrm{O}(1 \mathrm{~B})-\mathrm{C}(1 \mathrm{~B})-\mathrm{N}(1 \mathrm{~B})$ & $121.48(12)$ \\
\hline $\mathrm{O}(1 \mathrm{~B})-\mathrm{C}(1 \mathrm{~B})-\mathrm{C}(2 \mathrm{~B})$ & $122.30(12)$ \\
\hline $\mathrm{N}(1 \mathrm{~B})-\mathrm{C}(1 \mathrm{~B})-\mathrm{C}(2 \mathrm{~B})$ & $116.17(11)$ \\
\hline $\mathrm{C}(3 \mathrm{~B})-\mathrm{C}(2 \mathrm{~B})-\mathrm{C}(7 \mathrm{~B})$ & $120.84(12)$ \\
\hline $\mathrm{C}(3 \mathrm{~B})-\mathrm{C}(2 \mathrm{~B})-\mathrm{C}(1 \mathrm{~B})$ & $117.10(11)$ \\
\hline $\mathrm{C}(7 \mathrm{~B})-\mathrm{C}(2 \mathrm{~B})-\mathrm{C}(1 \mathrm{~B})$ & 121.91(11) \\
\hline $\mathrm{C}(4 \mathrm{~B})-\mathrm{C}(3 \mathrm{~B})-\mathrm{C}(2 \mathrm{~B})$ & $121.09(12)$ \\
\hline $\mathrm{C}(4 \mathrm{~B})-\mathrm{C}(3 \mathrm{~B})-\mathrm{H}(3 \mathrm{BA})$ & 119.5 \\
\hline $\mathrm{C}(2 \mathrm{~B})-\mathrm{C}(3 \mathrm{~B})-\mathrm{H}(3 \mathrm{BA})$ & 119.5 \\
\hline $\mathrm{C}(3 \mathrm{~B})-\mathrm{C}(4 \mathrm{~B})-\mathrm{C}(5 \mathrm{~B})$ & $118.94(12)$ \\
\hline $\mathrm{C}(3 \mathrm{~B})-\mathrm{C}(4 \mathrm{~B})-\mathrm{H}(4 \mathrm{BA})$ & 120.5 \\
\hline $\mathrm{C}(5 \mathrm{~B})-\mathrm{C}(4 \mathrm{~B})-\mathrm{H}(4 \mathrm{BA})$ & 120.5 \\
\hline
\end{tabular}




\begin{tabular}{|c|c|}
\hline$C(6 B)-C(5 B)-C(4 B)$ & $120.72(12)$ \\
\hline $\mathrm{C}(6 \mathrm{~B})-\mathrm{C}(5 \mathrm{~B})-\mathrm{H}(5 \mathrm{BA})$ & 119.6 \\
\hline $\mathrm{C}(4 \mathrm{~B})-\mathrm{C}(5 \mathrm{~B})-\mathrm{H}(5 \mathrm{BA})$ & 119.6 \\
\hline $\mathrm{C}(5 \mathrm{~B})-\mathrm{C}(6 \mathrm{~B})-\mathrm{C}(7 \mathrm{~B})$ & $121.53(12)$ \\
\hline$C(5 B)-C(6 B)-H(6 B A)$ & 119.2 \\
\hline$C(7 B)-C(6 B)-H(6 B A)$ & 119.2 \\
\hline $\mathrm{C}(2 \mathrm{~B})-\mathrm{C}(7 \mathrm{~B})-\mathrm{C}(6 \mathrm{~B})$ & $116.74(11)$ \\
\hline $\mathrm{C}(2 \mathrm{~B})-\mathrm{C}(7 \mathrm{~B})-\mathrm{C}(8 \mathrm{~B})$ & $118.80(11)$ \\
\hline$C(6 B)-C(7 B)-C(8 B)$ & $124.37(11)$ \\
\hline$C(13 B)-C(8 B)-C(9 B)$ & $117.26(10)$ \\
\hline $\mathrm{C}(13 \mathrm{~B})-\mathrm{C}(8 \mathrm{~B})-\mathrm{C}(7 \mathrm{~B})$ & $117.44(10)$ \\
\hline$C(9 B)-C(8 B)-C(7 B)$ & $125.30(10)$ \\
\hline$C(10 B)-C(9 B)-C(8 B)$ & $120.44(11)$ \\
\hline $\mathrm{C}(10 \mathrm{~B})-\mathrm{C}(9 \mathrm{~B})-\mathrm{N}(2 \mathrm{~B})$ & $119.83(11)$ \\
\hline $\mathrm{C}(8 \mathrm{~B})-\mathrm{C}(9 \mathrm{~B})-\mathrm{N}(2 \mathrm{~B})$ & $119.69(10)$ \\
\hline $\mathrm{C}(9 \mathrm{~B})-\mathrm{C}(10 \mathrm{~B})-\mathrm{C}(11 \mathrm{~B})$ & $120.46(11)$ \\
\hline $\mathrm{C}(9 \mathrm{~B})-\mathrm{C}(10 \mathrm{~B})-\mathrm{H}(10 \mathrm{~A})$ & 119.8 \\
\hline $\mathrm{C}(11 \mathrm{~B})-\mathrm{C}(10 \mathrm{~B})-\mathrm{H}(10 \mathrm{~A})$ & 119.8 \\
\hline $\mathrm{C}(12 \mathrm{~B})-\mathrm{C}(11 \mathrm{~B})-\mathrm{C}(10 \mathrm{~B})$ & $120.57(11)$ \\
\hline $\mathrm{C}(12 \mathrm{~B})-\mathrm{C}(11 \mathrm{~B})-\mathrm{H}(11 \mathrm{~A})$ & 119.7 \\
\hline$C(10 B)-C(11 B)-H(11 A)$ & 119.7 \\
\hline$C(11 B)-C(12 B)-C(13 B)$ & $120.18(11)$ \\
\hline $\mathrm{C}(11 \mathrm{~B})-\mathrm{C}(12 \mathrm{~B})-\mathrm{H}(12 \mathrm{~A})$ & 119.9 \\
\hline $\mathrm{C}(13 \mathrm{~B})-\mathrm{C}(12 \mathrm{~B})-\mathrm{H}(12 \mathrm{~A})$ & 119.9 \\
\hline $\mathrm{C}(12 \mathrm{~B})-\mathrm{C}(13 \mathrm{~B})-\mathrm{N}(1 \mathrm{~B})$ & $118.40(11)$ \\
\hline$C(12 B)-C(13 B)-C(8 B)$ & $120.68(11)$ \\
\hline$N(1 B)-C(13 B)-C(8 B)$ & $120.92(11)$ \\
\hline $\mathrm{N}(1 \mathrm{~B})-\mathrm{C}(14 \mathrm{~B})-\mathrm{H}(14 \mathrm{~A})$ & 109.5 \\
\hline $\mathrm{N}(1 \mathrm{~B})-\mathrm{C}(14 \mathrm{~B})-\mathrm{H}(14 \mathrm{~B})$ & 109.5 \\
\hline $\mathrm{H}(14 \mathrm{~A})-\mathrm{C}(14 \mathrm{~B})-\mathrm{H}(14 \mathrm{~B})$ & 109.5 \\
\hline $\mathrm{N}(1 \mathrm{~B})-\mathrm{C}(14 \mathrm{~B})-\mathrm{H}(14 \mathrm{C})$ & 109.5 \\
\hline $\mathrm{H}(14 \mathrm{~A})-\mathrm{C}(14 \mathrm{~B})-\mathrm{H}(14 \mathrm{C})$ & 109.5 \\
\hline $\mathrm{H}(14 \mathrm{~B})-\mathrm{C}(14 \mathrm{~B})-\mathrm{H}(14 \mathrm{C})$ & 109.5 \\
\hline$N(2 B)-C(15 B)-C(16 B)$ & $110.00(11)$ \\
\hline $\mathrm{N}(2 \mathrm{~B})-\mathrm{C}(15 \mathrm{~B})-\mathrm{H}(15 \mathrm{~A})$ & 109.7 \\
\hline$C(16 B)-C(15 B)-H(15 A)$ & 109.7 \\
\hline
\end{tabular}




\begin{tabular}{|c|c|}
\hline $\mathrm{N}(2 \mathrm{~B})-\mathrm{C}(15 \mathrm{~B})-\mathrm{H}(15 \mathrm{~B})$ & 109.7 \\
\hline $\mathrm{C}(16 \mathrm{~B})-\mathrm{C}(15 \mathrm{~B})-\mathrm{H}(15 \mathrm{~B})$ & 109.7 \\
\hline $\mathrm{H}(15 \mathrm{~A})-\mathrm{C}(15 \mathrm{~B})-\mathrm{H}(15 \mathrm{~B})$ & 108.2 \\
\hline $\mathrm{O}(2 \mathrm{~B})-\mathrm{C}(16 \mathrm{~B})-\mathrm{C}(15 \mathrm{~B})$ & $111.68(10)$ \\
\hline $\mathrm{O}(2 \mathrm{~B})-\mathrm{C}(16 \mathrm{~B})-\mathrm{H}(16 \mathrm{~A})$ & 109.3 \\
\hline $\mathrm{C}(15 \mathrm{~B})-\mathrm{C}(16 \mathrm{~B})-\mathrm{H}(16 \mathrm{~A})$ & 109.3 \\
\hline $\mathrm{O}(2 \mathrm{~B})-\mathrm{C}(16 \mathrm{~B})-\mathrm{H}(16 \mathrm{~B})$ & 109.3 \\
\hline$C(15 B)-C(16 B)-H(16 B)$ & 109.3 \\
\hline $\mathrm{H}(16 \mathrm{~A})-\mathrm{C}(16 \mathrm{~B})-\mathrm{H}(16 \mathrm{~B})$ & 107.9 \\
\hline $\mathrm{O}(2 \mathrm{~B})-\mathrm{C}(17 \mathrm{~B})-\mathrm{C}(18 \mathrm{~B})$ & $111.72(11)$ \\
\hline $\mathrm{O}(2 \mathrm{~B})-\mathrm{C}(17 \mathrm{~B})-\mathrm{H}(17 \mathrm{~A})$ & 109.3 \\
\hline $\mathrm{C}(18 \mathrm{~B})-\mathrm{C}(17 \mathrm{~B})-\mathrm{H}(17 \mathrm{~A})$ & 109.3 \\
\hline $\mathrm{O}(2 \mathrm{~B})-\mathrm{C}(17 \mathrm{~B})-\mathrm{H}(17 \mathrm{~B})$ & 109.3 \\
\hline $\mathrm{C}(18 \mathrm{~B})-\mathrm{C}(17 \mathrm{~B})-\mathrm{H}(17 \mathrm{~B})$ & 109.3 \\
\hline $\mathrm{H}(17 \mathrm{~A})-\mathrm{C}(17 \mathrm{~B})-\mathrm{H}(17 \mathrm{~B})$ & 107.9 \\
\hline $\mathrm{N}(2 \mathrm{~B})-\mathrm{C}(18 \mathrm{~B})-\mathrm{C}(17 \mathrm{~B})$ & $107.62(10)$ \\
\hline $\mathrm{N}(2 \mathrm{~B})-\mathrm{C}(18 \mathrm{~B})-\mathrm{H}(18 \mathrm{~A})$ & 110.2 \\
\hline $\mathrm{C}(17 \mathrm{~B})-\mathrm{C}(18 \mathrm{~B})-\mathrm{H}(18 \mathrm{~A})$ & 110.2 \\
\hline $\mathrm{N}(2 \mathrm{~B})-\mathrm{C}(18 \mathrm{~B})-\mathrm{H}(18 \mathrm{~B})$ & 110.2 \\
\hline $\mathrm{C}(17 \mathrm{~B})-\mathrm{C}(18 \mathrm{~B})-\mathrm{H}(18 \mathrm{~B})$ & 110.2 \\
\hline $\mathrm{H}(18 \mathrm{~A})-\mathrm{C}(18 \mathrm{~B})-\mathrm{H}(18 \mathrm{~B})$ & 108.5 \\
\hline $\mathrm{C}(17 \mathrm{~A})-\mathrm{O}(2 \mathrm{~A})-\mathrm{C}(16 \mathrm{~A})$ & $109.80(9)$ \\
\hline $\mathrm{C}(1 \mathrm{~A})-\mathrm{N}(1 \mathrm{~A})-\mathrm{C}(13 \mathrm{~A})$ & $123.95(11)$ \\
\hline$C(1 \mathrm{~A})-\mathrm{N}(1 \mathrm{~A})-\mathrm{C}(14 \mathrm{~A})$ & $117.00(11)$ \\
\hline$C(13 A)-N(1 A)-C(14 A)$ & $118.94(12)$ \\
\hline $\mathrm{C}(9 \mathrm{~A})-\mathrm{N}(2 \mathrm{~A})-\mathrm{C}(18 \mathrm{~A})$ & $116.88(10)$ \\
\hline $\mathrm{C}(9 \mathrm{~A})-\mathrm{N}(2 \mathrm{~A})-\mathrm{C}(15 \mathrm{~A})$ & $114.10(9)$ \\
\hline $\mathrm{C}(18 \mathrm{~A})-\mathrm{N}(2 \mathrm{~A})-\mathrm{C}(15 \mathrm{~A})$ & $109.09(9)$ \\
\hline $\mathrm{O}(1 \mathrm{~A})-\mathrm{C}(1 \mathrm{~A})-\mathrm{N}(1 \mathrm{~A})$ & $121.49(13)$ \\
\hline $\mathrm{O}(1 \mathrm{~A})-\mathrm{C}(1 \mathrm{~A})-\mathrm{C}(2 \mathrm{~A})$ & $122.47(13)$ \\
\hline $\mathrm{N}(1 \mathrm{~A})-\mathrm{C}(1 \mathrm{~A})-\mathrm{C}(2 \mathrm{~A})$ & $115.98(11)$ \\
\hline$C(3 A)-C(2 A)-C(7 A)$ & $121.00(12)$ \\
\hline $\mathrm{C}(3 \mathrm{~A})-\mathrm{C}(2 \mathrm{~A})-\mathrm{C}(1 \mathrm{~A})$ & $116.86(11)$ \\
\hline$C(7 A)-C(2 A)-C(1 A)$ & $121.99(12)$ \\
\hline$C(4 A)-C(3 A)-C(2 A)$ & $120.91(12)$ \\
\hline $\mathrm{C}(4 \mathrm{~A})-\mathrm{C}(3 \mathrm{~A})-\mathrm{H}(3 \mathrm{AA})$ & 119.5 \\
\hline
\end{tabular}




\begin{tabular}{|c|c|}
\hline $\mathrm{C}(2 \mathrm{~A})-\mathrm{C}(3 \mathrm{~A})-\mathrm{H}(3 \mathrm{AA})$ & 119.5 \\
\hline$C(3 \mathrm{~A})-\mathrm{C}(4 \mathrm{~A})-\mathrm{C}(5 \mathrm{~A})$ & $119.00(12)$ \\
\hline $\mathrm{C}(3 \mathrm{~A})-\mathrm{C}(4 \mathrm{~A})-\mathrm{H}(4 \mathrm{AA})$ & 120.5 \\
\hline $\mathrm{C}(5 \mathrm{~A})-\mathrm{C}(4 \mathrm{~A})-\mathrm{H}(4 \mathrm{AA})$ & 120.5 \\
\hline$C(6 A)-C(5 A)-C(4 A)$ & $120.68(12)$ \\
\hline$C(6 A)-C(5 A)-H(5 A A)$ & 119.7 \\
\hline $\mathrm{C}(4 \mathrm{~A})-\mathrm{C}(5 \mathrm{~A})-\mathrm{H}(5 \mathrm{AA})$ & 119.7 \\
\hline$C(5 A)-C(6 A)-C(7 A)$ & $121.70(12)$ \\
\hline$C(5 A)-C(6 A)-H(6 A A)$ & 119.2 \\
\hline$C(7 A)-C(6 A)-H(6 A A)$ & 119.2 \\
\hline$C(2 A)-C(7 A)-C(6 A)$ & $116.69(11)$ \\
\hline$C(2 A)-C(7 A)-C(8 A)$ & $118.88(11)$ \\
\hline$C(6 A)-C(7 A)-C(8 A)$ & $124.27(11)$ \\
\hline $\mathrm{C}(13 \mathrm{~A})-\mathrm{C}(8 \mathrm{~A})-\mathrm{C}(9 \mathrm{~A})$ & $117.04(11)$ \\
\hline$C(13 A)-C(8 A)-C(7 A)$ & $117.33(11)$ \\
\hline $\mathrm{C}(9 \mathrm{~A})-\mathrm{C}(8 \mathrm{~A})-\mathrm{C}(7 \mathrm{~A})$ & $125.56(11)$ \\
\hline $\mathrm{C}(10 \mathrm{~A})-\mathrm{C}(9 \mathrm{~A})-\mathrm{N}(2 \mathrm{~A})$ & $119.87(11)$ \\
\hline$C(10 A)-C(9 A)-C(8 A)$ & $120.01(11)$ \\
\hline $\mathrm{N}(2 \mathrm{~A})-\mathrm{C}(9 \mathrm{~A})-\mathrm{C}(8 \mathrm{~A})$ & $120.11(10)$ \\
\hline$C(11 A)-C(10 A)-C(9 A)$ & $120.85(12)$ \\
\hline$C(11 A)-C(10 A)-H(10 B)$ & 119.6 \\
\hline $\mathrm{C}(9 \mathrm{~A})-\mathrm{C}(10 \mathrm{~A})-\mathrm{H}(10 \mathrm{~B})$ & 119.6 \\
\hline$C(12 A)-C(11 A)-C(10 A)$ & $120.48(12)$ \\
\hline $\mathrm{C}(12 \mathrm{~A})-\mathrm{C}(11 \mathrm{~A})-\mathrm{H}(11 \mathrm{~B})$ & 119.8 \\
\hline$C(10 A)-C(11 A)-H(11 B)$ & 119.8 \\
\hline$C(11 A)-C(12 A)-C(13 A)$ & $120.01(12)$ \\
\hline $\mathrm{C}(11 \mathrm{~A})-\mathrm{C}(12 \mathrm{~A})-\mathrm{H}(12 \mathrm{~B})$ & 120.0 \\
\hline $\mathrm{C}(13 \mathrm{~A})-\mathrm{C}(12 \mathrm{~A})-\mathrm{H}(12 \mathrm{~B})$ & 120.0 \\
\hline $\mathrm{C}(12 \mathrm{~A})-\mathrm{C}(13 \mathrm{~A})-\mathrm{N}(1 \mathrm{~A})$ & $118.53(11)$ \\
\hline$C(12 A)-C(13 A)-C(8 A)$ & $121.03(12)$ \\
\hline$N(1 A)-C(13 A)-C(8 A)$ & $120.44(11)$ \\
\hline $\mathrm{N}(1 \mathrm{~A})-\mathrm{C}(14 \mathrm{~A})-\mathrm{H}(14 \mathrm{D})$ & 109.5 \\
\hline $\mathrm{N}(1 \mathrm{~A})-\mathrm{C}(14 \mathrm{~A})-\mathrm{H}(14 \mathrm{E})$ & 109.5 \\
\hline $\mathrm{H}(14 \mathrm{D})-\mathrm{C}(14 \mathrm{~A})-\mathrm{H}(14 \mathrm{E})$ & 109.5 \\
\hline $\mathrm{N}(1 \mathrm{~A})-\mathrm{C}(14 \mathrm{~A})-\mathrm{H}(14 \mathrm{~F})$ & 109.5 \\
\hline $\mathrm{H}(14 \mathrm{D})-\mathrm{C}(14 \mathrm{~A})-\mathrm{H}(14 \mathrm{~F})$ & 109.5 \\
\hline
\end{tabular}




$\begin{array}{ll}\mathrm{H}(14 \mathrm{E})-\mathrm{C}(14 \mathrm{~A})-\mathrm{H}(14 \mathrm{~F}) & 109.5 \\ \mathrm{~N}(2 \mathrm{~A})-\mathrm{C}(15 \mathrm{~A})-\mathrm{C}(16 \mathrm{~A}) & 110.28(10) \\ \mathrm{N}(2 \mathrm{~A})-\mathrm{C}(15 \mathrm{~A})-\mathrm{H}(15 \mathrm{C}) & 109.6 \\ \mathrm{C}(16 \mathrm{~A})-\mathrm{C}(15 \mathrm{~A})-\mathrm{H}(15 \mathrm{C}) & 109.6 \\ \mathrm{~N}(2 \mathrm{~A})-\mathrm{C}(15 \mathrm{~A})-\mathrm{H}(15 \mathrm{D}) & 109.6 \\ \mathrm{C}(16 \mathrm{~A})-\mathrm{C}(15 \mathrm{~A})-\mathrm{H}(15 \mathrm{D}) & 109.6 \\ \mathrm{H}(15 \mathrm{C})-\mathrm{C}(15 \mathrm{~A})-\mathrm{H}(15 \mathrm{D}) & 108.1 \\ \mathrm{O}(2 \mathrm{~A})-\mathrm{C}(16 \mathrm{~A})-\mathrm{C}(15 \mathrm{~A}) & 111.19(10) \\ \mathrm{O}(2 \mathrm{~A})-\mathrm{C}(16 \mathrm{~A})-\mathrm{H}(16 \mathrm{C}) & 109.4 \\ \mathrm{C}(15 \mathrm{~A})-\mathrm{C}(16 \mathrm{~A})-\mathrm{H}(16 \mathrm{C}) & 109.4 \\ \mathrm{O}(2 \mathrm{~A})-\mathrm{C}(16 \mathrm{~A})-\mathrm{H}(16 \mathrm{D}) & 109.4 \\ \mathrm{C}(15 \mathrm{~A})-\mathrm{C}(16 \mathrm{~A})-\mathrm{H}(16 \mathrm{D}) & 109.4 \\ \mathrm{H}(16 \mathrm{C})-\mathrm{C}(16 \mathrm{~A})-\mathrm{H}(16 \mathrm{D}) & 108.0 \\ \mathrm{O}(2 \mathrm{~A})-\mathrm{C}(17 \mathrm{~A})-\mathrm{C}(18 \mathrm{~A}) & 112.08(10) \\ \mathrm{O}(2 \mathrm{~A})-\mathrm{C}(17 \mathrm{~A})-\mathrm{H}(17 \mathrm{C}) & 109.2 \\ \mathrm{C}(18 \mathrm{~A})-\mathrm{C}(17 \mathrm{~A})-\mathrm{H}(17 \mathrm{C}) & 109.2 \\ \mathrm{O}(2 \mathrm{~A})-\mathrm{C}(17 \mathrm{~A})-\mathrm{H}(17 \mathrm{D}) & 109.2 \\ \mathrm{C}(18 \mathrm{~A})-\mathrm{C}(17 \mathrm{~A})-\mathrm{H}(17 \mathrm{D}) & 109.2 \\ \mathrm{H}(17 \mathrm{C})-\mathrm{C}(17 \mathrm{~A})-\mathrm{H}(17 \mathrm{D}) & 107.9 \\ \mathrm{~N}(2 \mathrm{~A})-\mathrm{C}(18 \mathrm{~A})-\mathrm{C}(17 \mathrm{~A}) & 108.56(10) \\ \mathrm{N}(2 \mathrm{~A})-\mathrm{C}(18 \mathrm{~A})-\mathrm{H}(18 \mathrm{C}) & 110.0 \\ \mathrm{C}(17 \mathrm{~A})-\mathrm{C}(18 \mathrm{~A})-\mathrm{H}(18 \mathrm{C}) & 110.0 \\ \mathrm{~N}(2 \mathrm{~A})-\mathrm{C}(18 \mathrm{~A})-\mathrm{H}(18 \mathrm{D}) & 110.0 \\ \mathrm{C}(17 \mathrm{~A})-\mathrm{C}(18 \mathrm{~A})-\mathrm{H}(18 \mathrm{D}) & 110.0 \\ \mathrm{H}(18 \mathrm{C})-\mathrm{C}(18 \mathrm{~A})-\mathrm{H}(18 \mathrm{D}) & 108.4 \\ & \\ & \end{array}$

Symmetry transformations used to generate equivalent atoms: 
Table 4. Anisotropic displacement parameters $\left(\AA^{2} \times 10^{3}\right)$ for d2089_a. The anisotropic displacement factor exponent takes the form: $-2 p^{2}\left[h^{2} a^{* 2} U^{11}+\ldots+2 h k a^{*} b^{*} U^{12}\right]$

\begin{tabular}{|c|c|c|c|c|c|c|}
\hline & $\mathrm{U}^{11}$ & $\mathrm{U}^{22}$ & $\mathrm{U}^{33}$ & $\mathrm{U}^{23}$ & $\mathrm{U}^{13}$ & $\mathrm{U}^{12}$ \\
\hline $\mathrm{O}(1 \mathrm{~B})$ & $24(1)$ & $40(1)$ & $50(1)$ & $0(1)$ & $-15(1)$ & $-6(1)$ \\
\hline $\mathrm{O}(2 \mathrm{~B})$ & $26(1)$ & $57(1)$ & $30(1)$ & $-2(1)$ & $-11(1)$ & $-11(1)$ \\
\hline $\mathrm{N}(1 \mathrm{~B})$ & $26(1)$ & $21(1)$ & $28(1)$ & $-3(1)$ & $-12(1)$ & $-4(1)$ \\
\hline $\mathrm{N}(2 \mathrm{~B})$ & $16(1)$ & $24(1)$ & $22(1)$ & $-4(1)$ & $-4(1)$ & $-4(1)$ \\
\hline $\mathrm{C}(1 \mathrm{~B})$ & $23(1)$ & $21(1)$ & $36(1)$ & $-6(1)$ & $-10(1)$ & $-5(1)$ \\
\hline $\mathrm{C}(2 \mathrm{~B})$ & $22(1)$ & $20(1)$ & $29(1)$ & $-7(1)$ & $-5(1)$ & $-7(1)$ \\
\hline$C(3 B)$ & $20(1)$ & $31(1)$ & $37(1)$ & $-10(1)$ & $-2(1)$ & $-9(1)$ \\
\hline $\mathrm{C}(4 \mathrm{~B})$ & $28(1)$ & $37(1)$ & $28(1)$ & $-8(1)$ & $2(1)$ & $-17(1)$ \\
\hline$C(5 B)$ & $33(1)$ & $30(1)$ & $22(1)$ & $-4(1)$ & $-5(1)$ & $-14(1)$ \\
\hline $\mathrm{C}(6 \mathrm{~B})$ & $22(1)$ & $24(1)$ & $23(1)$ & $-5(1)$ & $-5(1)$ & $-8(1)$ \\
\hline $\mathrm{C}(7 \mathrm{~B})$ & $21(1)$ & $18(1)$ & $23(1)$ & $-6(1)$ & $-4(1)$ & $-6(1)$ \\
\hline $\mathrm{C}(8 \mathrm{~B})$ & $21(1)$ & $15(1)$ & $21(1)$ & $-6(1)$ & $-4(1)$ & $-4(1)$ \\
\hline $\mathrm{C}(9 \mathrm{~B})$ & $21(1)$ & $16(1)$ & $22(1)$ & $-5(1)$ & $-4(1)$ & $-3(1)$ \\
\hline $\mathrm{C}(10 \mathrm{~B})$ & $20(1)$ & $24(1)$ & $26(1)$ & $-6(1)$ & $-1(1)$ & $-6(1)$ \\
\hline $\mathrm{C}(11 \mathrm{~B})$ & $31(1)$ & $26(1)$ & $21(1)$ & $-5(1)$ & 1(1) & $-10(1)$ \\
\hline$C(12 B)$ & $34(1)$ & $20(1)$ & $20(1)$ & $-3(1)$ & $-7(1)$ & $-9(1)$ \\
\hline $\mathrm{C}(13 \mathrm{~B})$ & $23(1)$ & $16(1)$ & $25(1)$ & $-6(1)$ & $-7(1)$ & $-4(1)$ \\
\hline $\mathrm{C}(14 \mathrm{~B})$ & $34(1)$ & $34(1)$ & $33(1)$ & $-1(1)$ & $-17(1)$ & $-2(1)$ \\
\hline$C(15 B)$ & $24(1)$ & $30(1)$ & $25(1)$ & $-8(1)$ & $-6(1)$ & $-5(1)$ \\
\hline$C(16 B)$ & $25(1)$ & $52(1)$ & $27(1)$ & $-6(1)$ & $-6(1)$ & $-9(1)$ \\
\hline $\mathrm{C}(17 \mathrm{~B})$ & $21(1)$ & $38(1)$ & $38(1)$ & $5(1)$ & $-10(1)$ & $-6(1)$ \\
\hline $\mathrm{C}(18 \mathrm{~B})$ & $18(1)$ & $28(1)$ & $30(1)$ & $-3(1)$ & $-4(1)$ & $-5(1)$ \\
\hline $\mathrm{O}(1 \mathrm{~A})$ & $28(1)$ & $49(1)$ & $58(1)$ & $-21(1)$ & $8(1)$ & $-4(1)$ \\
\hline $\mathrm{O}(2 \mathrm{~A})$ & $28(1)$ & $38(1)$ & $29(1)$ & $-12(1)$ & $3(1)$ & $-10(1)$ \\
\hline $\mathrm{N}(1 \mathrm{~A})$ & $32(1)$ & $26(1)$ & $23(1)$ & $-6(1)$ & 1(1) & $-4(1)$ \\
\hline $\mathrm{N}(2 \mathrm{~A})$ & $17(1)$ & $22(1)$ & $22(1)$ & $-4(1)$ & $-4(1)$ & $-4(1)$ \\
\hline $\mathrm{C}(1 \mathrm{~A})$ & $26(1)$ & $28(1)$ & $28(1)$ & $-1(1)$ & 1(1) & $-5(1)$ \\
\hline $\mathrm{C}(2 \mathrm{~A})$ & $23(1)$ & $25(1)$ & $22(1)$ & $2(1)$ & $-4(1)$ & $-5(1)$ \\
\hline $\mathrm{C}(3 \mathrm{~A})$ & $20(1)$ & $38(1)$ & $29(1)$ & 1(1) & $-4(1)$ & $-8(1)$ \\
\hline$C(4 A)$ & $28(1)$ & $41(1)$ & $31(1)$ & $0(1)$ & $-10(1)$ & $-17(1)$ \\
\hline$C(5 A)$ & $30(1)$ & $29(1)$ & $25(1)$ & $-2(1)$ & $-8(1)$ & $-11(1)$ \\
\hline
\end{tabular}




\begin{tabular}{lllllll}
$\mathrm{C}(6 \mathrm{~A})$ & $23(1)$ & $24(1)$ & $22(1)$ & $-1(1)$ & $-4(1)$ & $-7(1)$ \\
$\mathrm{C}(7 \mathrm{~A})$ & $21(1)$ & $21(1)$ & $18(1)$ & $2(1)$ & $-5(1)$ & $-5(1)$ \\
$\mathrm{C}(8 \mathrm{~A})$ & $23(1)$ & $17(1)$ & $18(1)$ & $1(1)$ & $-6(1)$ & $-4(1)$ \\
$\mathrm{C}(9 \mathrm{~A})$ & $24(1)$ & $17(1)$ & $20(1)$ & $1(1)$ & $-8(1)$ & $-5(1)$ \\
$\mathrm{C}(10 \mathrm{~A})$ & $26(1)$ & $25(1)$ & $24(1)$ & $1(1)$ & $-10(1)$ & $-8(1)$ \\
$\mathrm{C}(11 \mathrm{~A})$ & $39(1)$ & $25(1)$ & $26(1)$ & $1(1)$ & $-16(1)$ & $-12(1)$ \\
$\mathrm{C}(12 \mathrm{~A})$ & $44(1)$ & $21(1)$ & $20(1)$ & $-3(1)$ & $-10(1)$ & $-8(1)$ \\
$\mathrm{C}(13 \mathrm{~A})$ & $29(1)$ & $19(1)$ & $18(1)$ & $2(1)$ & $-5(1)$ & $-4(1)$ \\
$\mathrm{C}(14 \mathrm{~A})$ & $45(1)$ & $46(1)$ & $36(1)$ & $-22(1)$ & $4(1)$ & $-6(1)$ \\
$\mathrm{C}(15 \mathrm{~A})$ & $23(1)$ & $23(1)$ & $22(1)$ & $-2(1)$ & $-5(1)$ & $-5(1)$ \\
$\mathrm{C}(16 \mathrm{~A})$ & $28(1)$ & $33(1)$ & $25(1)$ & $-7(1)$ & $-4(1)$ & $-9(1)$ \\
$\mathrm{C}(17 \mathrm{~A})$ & $21(1)$ & $28(1)$ & $39(1)$ & $-12(1)$ & $-1(1)$ & $-6(1)$ \\
$\mathrm{C}(18 \mathrm{~A})$ & $18(1)$ & $25(1)$ & $34(1)$ & $-7(1)$ & $-5(1)$ & $-6(1)$ \\
& & & & & & \\
\hline
\end{tabular}


Table 5. Hydrogen coordinates ( x 104) and isotropic displacement parameters $\left(\AA^{2} \times 10^{3}\right)$ for d2089_a.

\begin{tabular}{|c|c|c|c|c|}
\hline & $\mathrm{x}$ & $\mathrm{y}$ & $\mathrm{z}$ & $\mathrm{U}(\mathrm{eq})$ \\
\hline $\mathrm{H}(3 \mathrm{BA})$ & 5437 & 1100 & 7497 & 35 \\
\hline $\mathrm{H}(4 \mathrm{BA})$ & 6030 & -210 & 9019 & 36 \\
\hline $\mathrm{H}(5 \mathrm{BA})$ & 8467 & -1015 & 9075 & 33 \\
\hline $\mathrm{H}(6 \mathrm{BA})$ & 10259 & -411 & 7689 & 27 \\
\hline $\mathrm{H}(10 \mathrm{~A})$ & 13488 & 992 & 4782 & 29 \\
\hline $\mathrm{H}(11 \mathrm{~A})$ & 12692 & 1860 & 3192 & 32 \\
\hline $\mathrm{H}(12 \mathrm{~A})$ & 10258 & 2397 & 3218 & 29 \\
\hline $\mathrm{H}(14 \mathrm{~A})$ & 8227 & 2166 & 3185 & 51 \\
\hline $\mathrm{H}(14 \mathrm{~B})$ & 7898 & 3397 & 3577 & 51 \\
\hline $\mathrm{H}(14 \mathrm{C})$ & 6653 & 2733 & 3864 & 51 \\
\hline $\mathrm{H}(15 \mathrm{~A})$ & 10472 & 1697 & 7534 & 31 \\
\hline $\mathrm{H}(15 \mathrm{~B})$ & 12027 & 1962 & 7136 & 31 \\
\hline $\mathrm{H}(16 \mathrm{~A})$ & 11542 & 1370 & 9024 & 41 \\
\hline $\mathrm{H}(16 \mathrm{~B})$ & 11243 & 189 & 8906 & 41 \\
\hline $\mathrm{H}(17 \mathrm{~A})$ & 13223 & -1270 & 8063 & 41 \\
\hline $\mathrm{H}(17 \mathrm{~B})$ & 14808 & -1070 & 7722 & 41 \\
\hline $\mathrm{H}(18 \mathrm{~A})$ & 14100 & 512 & 6335 & 32 \\
\hline $\mathrm{H}(18 \mathrm{~B})$ & 13903 & -689 & 6157 & 32 \\
\hline $\mathrm{H}(3 \mathrm{AA})$ & 438 & 5547 & 9033 & 37 \\
\hline $\mathrm{H}(4 \mathrm{AA})$ & 1182 & 4008 & 8057 & 38 \\
\hline $\mathrm{H}(5 \mathrm{AA})$ & 3647 & 3291 & 7303 & 32 \\
\hline $\mathrm{H}(6 \mathrm{AA})$ & 5321 & 4144 & 7475 & 28 \\
\hline $\mathrm{H}(10 \mathrm{~B})$ & 8344 & 5914 & 8122 & 30 \\
\hline $\mathrm{H}(11 \mathrm{~B})$ & 7431 & 6972 & 9554 & 34 \\
\hline $\mathrm{H}(12 \mathrm{~B})$ & 4967 & 7522 & 10281 & 34 \\
\hline $\mathrm{H}(14 \mathrm{D})$ & 2780 & 7397 & 11292 & 66 \\
\hline $\mathrm{H}(14 \mathrm{E})$ & 1338 & 7986 & 10852 & 66 \\
\hline $\mathrm{H}(14 \mathrm{~F})$ & 2720 & 8498 & 10324 & 66 \\
\hline $\mathrm{H}(15 \mathrm{C})$ & 6773 & 6534 & 5875 & 28 \\
\hline $\mathrm{H}(15 \mathrm{D})$ & 5341 & 6082 & 6389 & 28 \\
\hline
\end{tabular}




\begin{tabular}{lllll}
$\mathrm{H}(16 \mathrm{C})$ & 6418 & 4387 & 5578 & 35 \\
$\mathrm{H}(16 \mathrm{D})$ & 6469 & 5530 & 4660 & 35 \\
$\mathrm{H}(17 \mathrm{C})$ & 9996 & 3717 & 5677 & 36 \\
$\mathrm{H}(17 \mathrm{D})$ & 8534 & 3315 & 6209 & 36 \\
$\mathrm{H}(18 \mathrm{C})$ & 8971 & 4282 & 7402 & 31 \\
$\mathrm{H}(18 \mathrm{D})$ & 8984 & 5436 & 6488 & 31 \\
\hline
\end{tabular}


Table 6. Torsion angles $\left[{ }^{\circ}\right]$ for d2089_a.

\begin{tabular}{|c|c|}
\hline $\mathrm{C}(13 \mathrm{~B})-\mathrm{N}(1 \mathrm{~B})-\mathrm{C}(1 \mathrm{~B})-\mathrm{O}(1 \mathrm{~B})$ & $174.78(11)$ \\
\hline$C(14 B)-N(1 B)-C(1 B)-O(1 B)$ & $-1.81(18)$ \\
\hline $\mathrm{C}(13 \mathrm{~B})-\mathrm{N}(1 \mathrm{~B})-\mathrm{C}(1 \mathrm{~B})-\mathrm{C}(2 \mathrm{~B})$ & $-7.88(17)$ \\
\hline $\mathrm{C}(14 \mathrm{~B})-\mathrm{N}(1 \mathrm{~B})-\mathrm{C}(1 \mathrm{~B})-\mathrm{C}(2 \mathrm{~B})$ & $175.54(11)$ \\
\hline $\mathrm{O}(1 \mathrm{~B})-\mathrm{C}(1 \mathrm{~B})-\mathrm{C}(2 \mathrm{~B})-\mathrm{C}(3 \mathrm{~B})$ & $5.20(19)$ \\
\hline $\mathrm{N}(1 \mathrm{~B})-\mathrm{C}(1 \mathrm{~B})-\mathrm{C}(2 \mathrm{~B})-\mathrm{C}(3 \mathrm{~B})$ & $-172.12(11)$ \\
\hline $\mathrm{O}(1 \mathrm{~B})-\mathrm{C}(1 \mathrm{~B})-\mathrm{C}(2 \mathrm{~B})-\mathrm{C}(7 \mathrm{~B})$ & $-179.25(12)$ \\
\hline$N(1 B)-C(1 B)-C(2 B)-C(7 B)$ & $3.43(17)$ \\
\hline $\mathrm{C}(7 \mathrm{~B})-\mathrm{C}(2 \mathrm{~B})-\mathrm{C}(3 \mathrm{~B})-\mathrm{C}(4 \mathrm{~B})$ & $-3.52(19)$ \\
\hline $\mathrm{C}(1 \mathrm{~B})-\mathrm{C}(2 \mathrm{~B})-\mathrm{C}(3 \mathrm{~B})-\mathrm{C}(4 \mathrm{~B})$ & $172.09(12)$ \\
\hline $\mathrm{C}(2 \mathrm{~B})-\mathrm{C}(3 \mathrm{~B})-\mathrm{C}(4 \mathrm{~B})-\mathrm{C}(5 \mathrm{~B})$ & $0.1(2)$ \\
\hline $\mathrm{C}(3 \mathrm{~B})-\mathrm{C}(4 \mathrm{~B})-\mathrm{C}(5 \mathrm{~B})-\mathrm{C}(6 \mathrm{~B})$ & $2.63(19)$ \\
\hline$C(4 B)-C(5 B)-C(6 B)-C(7 B)$ & $-1.98(19)$ \\
\hline $\mathrm{C}(3 \mathrm{~B})-\mathrm{C}(2 \mathrm{~B})-\mathrm{C}(7 \mathrm{~B})-\mathrm{C}(6 \mathrm{~B})$ & $4.06(17)$ \\
\hline$C(1 B)-C(2 B)-C(7 B)-C(6 B)$ & $-171.34(11)$ \\
\hline $\mathrm{C}(3 \mathrm{~B})-\mathrm{C}(2 \mathrm{~B})-\mathrm{C}(7 \mathrm{~B})-\mathrm{C}(8 \mathrm{~B})$ & $-179.35(11)$ \\
\hline $\mathrm{C}(1 \mathrm{~B})-\mathrm{C}(2 \mathrm{~B})-\mathrm{C}(7 \mathrm{~B})-\mathrm{C}(8 \mathrm{~B})$ & $5.25(17)$ \\
\hline$C(5 B)-C(6 B)-C(7 B)-C(2 B)$ & $-1.36(17)$ \\
\hline $\mathrm{C}(5 \mathrm{~B})-\mathrm{C}(6 \mathrm{~B})-\mathrm{C}(7 \mathrm{~B})-\mathrm{C}(8 \mathrm{~B})$ & $-177.74(11)$ \\
\hline $\mathrm{C}(2 \mathrm{~B})-\mathrm{C}(7 \mathrm{~B})-\mathrm{C}(8 \mathrm{~B})-\mathrm{C}(13 \mathrm{~B})$ & $-9.76(16)$ \\
\hline$C(6 B)-C(7 B)-C(8 B)-C(13 B)$ & $166.55(11)$ \\
\hline $\mathrm{C}(2 \mathrm{~B})-\mathrm{C}(7 \mathrm{~B})-\mathrm{C}(8 \mathrm{~B})-\mathrm{C}(9 \mathrm{~B})$ & $170.29(11)$ \\
\hline $\mathrm{C}(6 \mathrm{~B})-\mathrm{C}(7 \mathrm{~B})-\mathrm{C}(8 \mathrm{~B})-\mathrm{C}(9 \mathrm{~B})$ & $-13.40(18)$ \\
\hline$C(13 B)-C(8 B)-C(9 B)-C(10 B)$ & $-7.12(16)$ \\
\hline $\mathrm{C}(7 \mathrm{~B})-\mathrm{C}(8 \mathrm{~B})-\mathrm{C}(9 \mathrm{~B})-\mathrm{C}(10 \mathrm{~B})$ & $172.83(11)$ \\
\hline $\mathrm{C}(13 \mathrm{~B})-\mathrm{C}(8 \mathrm{~B})-\mathrm{C}(9 \mathrm{~B})-\mathrm{N}(2 \mathrm{~B})$ & $170.64(10)$ \\
\hline $\mathrm{C}(7 \mathrm{~B})-\mathrm{C}(8 \mathrm{~B})-\mathrm{C}(9 \mathrm{~B})-\mathrm{N}(2 \mathrm{~B})$ & $-9.41(17)$ \\
\hline $\mathrm{C}(18 \mathrm{~B})-\mathrm{N}(2 \mathrm{~B})-\mathrm{C}(9 \mathrm{~B})-\mathrm{C}(10 \mathrm{~B})$ & $-22.00(16)$ \\
\hline $\mathrm{C}(15 \mathrm{~B})-\mathrm{N}(2 \mathrm{~B})-\mathrm{C}(9 \mathrm{~B})-\mathrm{C}(10 \mathrm{~B})$ & $105.51(12)$ \\
\hline $\mathrm{C}(18 \mathrm{~B})-\mathrm{N}(2 \mathrm{~B})-\mathrm{C}(9 \mathrm{~B})-\mathrm{C}(8 \mathrm{~B})$ & $160.23(10)$ \\
\hline $\mathrm{C}(15 \mathrm{~B})-\mathrm{N}(2 \mathrm{~B})-\mathrm{C}(9 \mathrm{~B})-\mathrm{C}(8 \mathrm{~B})$ & $-72.26(13)$ \\
\hline$C(8 B)-C(9 B)-C(10 B)-C(11 B)$ & $3.79(18)$ \\
\hline $\mathrm{N}(2 \mathrm{~B})-\mathrm{C}(9 \mathrm{~B})-\mathrm{C}(10 \mathrm{~B})-\mathrm{C}(11 \mathrm{~B})$ & $-173.96(11)$ \\
\hline $\mathrm{C}(9 \mathrm{~B})-\mathrm{C}(10 \mathrm{~B})-\mathrm{C}(11 \mathrm{~B})-\mathrm{C}(12 \mathrm{~B})$ & $1.99(19)$ \\
\hline
\end{tabular}




\begin{tabular}{|c|c|}
\hline $\mathrm{C}(10 \mathrm{~B})-\mathrm{C}(11 \mathrm{~B})-\mathrm{C}(12 \mathrm{~B})-\mathrm{C}(13 \mathrm{~B})$ & $-4.16(19)$ \\
\hline $\mathrm{C}(11 \mathrm{~B})-\mathrm{C}(12 \mathrm{~B})-\mathrm{C}(13 \mathrm{~B})-\mathrm{N}(1 \mathrm{~B})$ & $179.76(11)$ \\
\hline $\mathrm{C}(11 \mathrm{~B})-\mathrm{C}(12 \mathrm{~B})-\mathrm{C}(13 \mathrm{~B})-\mathrm{C}(8 \mathrm{~B})$ & $0.55(18)$ \\
\hline $\mathrm{C}(1 \mathrm{~B})-\mathrm{N}(1 \mathrm{~B})-\mathrm{C}(13 \mathrm{~B})-\mathrm{C}(12 \mathrm{~B})$ & $-175.93(11)$ \\
\hline $\mathrm{C}(14 \mathrm{~B})-\mathrm{N}(1 \mathrm{~B})-\mathrm{C}(13 \mathrm{~B})-\mathrm{C}(12 \mathrm{~B})$ & $0.58(16)$ \\
\hline $\mathrm{C}(1 \mathrm{~B})-\mathrm{N}(1 \mathrm{~B})-\mathrm{C}(13 \mathrm{~B})-\mathrm{C}(8 \mathrm{~B})$ & $3.27(17)$ \\
\hline $\mathrm{C}(14 \mathrm{~B})-\mathrm{N}(1 \mathrm{~B})-\mathrm{C}(13 \mathrm{~B})-\mathrm{C}(8 \mathrm{~B})$ & $179.78(11)$ \\
\hline $\mathrm{C}(9 \mathrm{~B})-\mathrm{C}(8 \mathrm{~B})-\mathrm{C}(13 \mathrm{~B})-\mathrm{C}(12 \mathrm{~B})$ & $4.98(16)$ \\
\hline $\mathrm{C}(7 \mathrm{~B})-\mathrm{C}(8 \mathrm{~B})-\mathrm{C}(13 \mathrm{~B})-\mathrm{C}(12 \mathrm{~B})$ & $-174.98(10)$ \\
\hline $\mathrm{C}(9 \mathrm{~B})-\mathrm{C}(8 \mathrm{~B})-\mathrm{C}(13 \mathrm{~B})-\mathrm{N}(1 \mathrm{~B})$ & $-174.21(10)$ \\
\hline $\mathrm{C}(7 \mathrm{~B})-\mathrm{C}(8 \mathrm{~B})-\mathrm{C}(13 \mathrm{~B})-\mathrm{N}(1 \mathrm{~B})$ & $5.83(16)$ \\
\hline $\mathrm{C}(9 \mathrm{~B})-\mathrm{N}(2 \mathrm{~B})-\mathrm{C}(15 \mathrm{~B})-\mathrm{C}(16 \mathrm{~B})$ & $169.91(10)$ \\
\hline $\mathrm{C}(18 \mathrm{~B})-\mathrm{N}(2 \mathrm{~B})-\mathrm{C}(15 \mathrm{~B})-\mathrm{C}(16 \mathrm{~B})$ & $-58.45(13)$ \\
\hline $\mathrm{C}(17 \mathrm{~B})-\mathrm{O}(2 \mathrm{~B})-\mathrm{C}(16 \mathrm{~B})-\mathrm{C}(15 \mathrm{~B})$ & $-55.76(15)$ \\
\hline $\mathrm{N}(2 \mathrm{~B})-\mathrm{C}(15 \mathrm{~B})-\mathrm{C}(16 \mathrm{~B})-\mathrm{O}(2 \mathrm{~B})$ & $56.11(15)$ \\
\hline $\mathrm{C}(16 \mathrm{~B})-\mathrm{O}(2 \mathrm{~B})-\mathrm{C}(17 \mathrm{~B})-\mathrm{C}(18 \mathrm{~B})$ & $58.95(14)$ \\
\hline $\mathrm{C}(9 \mathrm{~B})-\mathrm{N}(2 \mathrm{~B})-\mathrm{C}(18 \mathrm{~B})-\mathrm{C}(17 \mathrm{~B})$ & $-170.73(10)$ \\
\hline $\mathrm{C}(15 \mathrm{~B})-\mathrm{N}(2 \mathrm{~B})-\mathrm{C}(18 \mathrm{~B})-\mathrm{C}(17 \mathrm{~B})$ & $60.08(13)$ \\
\hline $\mathrm{O}(2 \mathrm{~B})-\mathrm{C}(17 \mathrm{~B})-\mathrm{C}(18 \mathrm{~B})-\mathrm{N}(2 \mathrm{~B})$ & $-61.22(14)$ \\
\hline $\mathrm{C}(13 \mathrm{~A})-\mathrm{N}(1 \mathrm{~A})-\mathrm{C}(1 \mathrm{~A})-\mathrm{O}(1 \mathrm{~A})$ & $-173.90(12)$ \\
\hline $\mathrm{C}(14 \mathrm{~A})-\mathrm{N}(1 \mathrm{~A})-\mathrm{C}(1 \mathrm{~A})-\mathrm{O}(1 \mathrm{~A})$ & $2.16(19)$ \\
\hline $\mathrm{C}(13 \mathrm{~A})-\mathrm{N}(1 \mathrm{~A})-\mathrm{C}(1 \mathrm{~A})-\mathrm{C}(2 \mathrm{~A})$ & $9.06(17)$ \\
\hline $\mathrm{C}(14 \mathrm{~A})-\mathrm{N}(1 \mathrm{~A})-\mathrm{C}(1 \mathrm{~A})-\mathrm{C}(2 \mathrm{~A})$ & $-174.88(11)$ \\
\hline $\mathrm{O}(1 \mathrm{~A})-\mathrm{C}(1 \mathrm{~A})-\mathrm{C}(2 \mathrm{~A})-\mathrm{C}(3 \mathrm{~A})$ & $-9.00(19)$ \\
\hline $\mathrm{N}(1 \mathrm{~A})-\mathrm{C}(1 \mathrm{~A})-\mathrm{C}(2 \mathrm{~A})-\mathrm{C}(3 \mathrm{~A})$ & $168.02(11)$ \\
\hline $\mathrm{O}(1 \mathrm{~A})-\mathrm{C}(1 \mathrm{~A})-\mathrm{C}(2 \mathrm{~A})-\mathrm{C}(7 \mathrm{~A})$ & $175.46(12)$ \\
\hline $\mathrm{N}(1 \mathrm{~A})-\mathrm{C}(1 \mathrm{~A})-\mathrm{C}(2 \mathrm{~A})-\mathrm{C}(7 \mathrm{~A})$ & $-7.53(17)$ \\
\hline$C(7 A)-C(2 A)-C(3 A)-C(4 A)$ & $1.65(19)$ \\
\hline $\mathrm{C}(1 \mathrm{~A})-\mathrm{C}(2 \mathrm{~A})-\mathrm{C}(3 \mathrm{~A})-\mathrm{C}(4 \mathrm{~A})$ & $-173.94(12)$ \\
\hline $\mathrm{C}(2 \mathrm{~A})-\mathrm{C}(3 \mathrm{~A})-\mathrm{C}(4 \mathrm{~A})-\mathrm{C}(5 \mathrm{~A})$ & $0.15(19)$ \\
\hline$C(3 A)-C(4 A)-C(5 A)-C(6 A)$ & $-1.28(19)$ \\
\hline $\mathrm{C}(4 \mathrm{~A})-\mathrm{C}(5 \mathrm{~A})-\mathrm{C}(6 \mathrm{~A})-\mathrm{C}(7 \mathrm{~A})$ & $0.63(18)$ \\
\hline $\mathrm{C}(3 \mathrm{~A})-\mathrm{C}(2 \mathrm{~A})-\mathrm{C}(7 \mathrm{~A})-\mathrm{C}(6 \mathrm{~A})$ & $-2.23(17)$ \\
\hline $\mathrm{C}(1 \mathrm{~A})-\mathrm{C}(2 \mathrm{~A})-\mathrm{C}(7 \mathrm{~A})-\mathrm{C}(6 \mathrm{~A})$ & $173.13(11)$ \\
\hline $\mathrm{C}(3 \mathrm{~A})-\mathrm{C}(2 \mathrm{~A})-\mathrm{C}(7 \mathrm{~A})-\mathrm{C}(8 \mathrm{~A})$ & $-177.79(11)$ \\
\hline $\mathrm{C}(1 \mathrm{~A})-\mathrm{C}(2 \mathrm{~A})-\mathrm{C}(7 \mathrm{~A})-\mathrm{C}(8 \mathrm{~A})$ & $-2.43(17)$ \\
\hline
\end{tabular}




\begin{tabular}{|c|c|}
\hline$C(5 A)-C(6 A)-C(7 A)-C(2 A)$ & $1.11(17)$ \\
\hline$C(5 A)-C(6 A)-C(7 A)-C(8 A)$ & $176.41(11)$ \\
\hline $\mathrm{C}(2 \mathrm{~A})-\mathrm{C}(7 \mathrm{~A})-\mathrm{C}(8 \mathrm{~A})-\mathrm{C}(13 \mathrm{~A})$ & $11.11(16)$ \\
\hline $\mathrm{C}(6 \mathrm{~A})-\mathrm{C}(7 \mathrm{~A})-\mathrm{C}(8 \mathrm{~A})-\mathrm{C}(13 \mathrm{~A})$ & $-164.09(11)$ \\
\hline $\mathrm{C}(2 \mathrm{~A})-\mathrm{C}(7 \mathrm{~A})-\mathrm{C}(8 \mathrm{~A})-\mathrm{C}(9 \mathrm{~A})$ & $-172.10(11)$ \\
\hline$C(6 A)-C(7 A)-C(8 A)-C(9 A)$ & $12.70(18)$ \\
\hline $\mathrm{C}(18 \mathrm{~A})-\mathrm{N}(2 \mathrm{~A})-\mathrm{C}(9 \mathrm{~A})-\mathrm{C}(10 \mathrm{~A})$ & $13.86(16)$ \\
\hline $\mathrm{C}(15 \mathrm{~A})-\mathrm{N}(2 \mathrm{~A})-\mathrm{C}(9 \mathrm{~A})-\mathrm{C}(10 \mathrm{~A})$ & $-115.08(12)$ \\
\hline $\mathrm{C}(18 \mathrm{~A})-\mathrm{N}(2 \mathrm{~A})-\mathrm{C}(9 \mathrm{~A})-\mathrm{C}(8 \mathrm{~A})$ & $-166.41(10)$ \\
\hline$C(15 A)-N(2 A)-C(9 A)-C(8 A)$ & $64.65(14)$ \\
\hline$C(13 A)-C(8 A)-C(9 A)-C(10 A)$ & $8.84(16)$ \\
\hline$C(7 A)-C(8 A)-C(9 A)-C(10 A)$ & $-167.96(11)$ \\
\hline$C(13 A)-C(8 A)-C(9 A)-N(2 A)$ & $-170.90(10)$ \\
\hline $\mathrm{C}(7 \mathrm{~A})-\mathrm{C}(8 \mathrm{~A})-\mathrm{C}(9 \mathrm{~A})-\mathrm{N}(2 \mathrm{~A})$ & $12.30(17)$ \\
\hline$N(2 A)-C(9 A)-C(10 A)-C(11 A)$ & $174.67(11)$ \\
\hline$C(8 A)-C(9 A)-C(10 A)-C(11 A)$ & $-5.06(18)$ \\
\hline$C(9 A)-C(10 A)-C(11 A)-C(12 A)$ & $-1.55(19)$ \\
\hline $\mathrm{C}(10 \mathrm{~A})-\mathrm{C}(11 \mathrm{~A})-\mathrm{C}(12 \mathrm{~A})-\mathrm{C}(13 \mathrm{~A})$ & $4.02(19)$ \\
\hline$C(11 A)-C(12 A)-C(13 A)-N(1 A)$ & $-179.25(11)$ \\
\hline$C(11 A)-C(12 A)-C(13 A)-C(8 A)$ & $0.14(18)$ \\
\hline $\mathrm{C}(1 \mathrm{~A})-\mathrm{N}(1 \mathrm{~A})-\mathrm{C}(13 \mathrm{~A})-\mathrm{C}(12 \mathrm{~A})$ & $179.09(11)$ \\
\hline $\mathrm{C}(14 \mathrm{~A})-\mathrm{N}(1 \mathrm{~A})-\mathrm{C}(13 \mathrm{~A})-\mathrm{C}(12 \mathrm{~A})$ & $3.10(17)$ \\
\hline $\mathrm{C}(1 \mathrm{~A})-\mathrm{N}(1 \mathrm{~A})-\mathrm{C}(13 \mathrm{~A})-\mathrm{C}(8 \mathrm{~A})$ & $-0.30(18)$ \\
\hline$C(14 A)-N(1 A)-C(13 A)-C(8 A)$ & $-176.29(12)$ \\
\hline $\mathrm{C}(9 \mathrm{~A})-\mathrm{C}(8 \mathrm{~A})-\mathrm{C}(13 \mathrm{~A})-\mathrm{C}(12 \mathrm{~A})$ & $-6.45(16)$ \\
\hline$C(7 A)-C(8 A)-C(13 A)-C(12 A)$ & $170.62(11)$ \\
\hline$C(9 A)-C(8 A)-C(13 A)-N(1 A)$ & $172.92(10)$ \\
\hline$C(7 A)-C(8 A)-C(13 A)-N(1 A)$ & $-10.01(16)$ \\
\hline $\mathrm{C}(9 \mathrm{~A})-\mathrm{N}(2 \mathrm{~A})-\mathrm{C}(15 \mathrm{~A})-\mathrm{C}(16 \mathrm{~A})$ & $-169.59(10)$ \\
\hline $\mathrm{C}(18 \mathrm{~A})-\mathrm{N}(2 \mathrm{~A})-\mathrm{C}(15 \mathrm{~A})-\mathrm{C}(16 \mathrm{~A})$ & $57.65(13)$ \\
\hline $\mathrm{C}(17 \mathrm{~A})-\mathrm{O}(2 \mathrm{~A})-\mathrm{C}(16 \mathrm{~A})-\mathrm{C}(15 \mathrm{~A})$ & $57.42(13)$ \\
\hline $\mathrm{N}(2 \mathrm{~A})-\mathrm{C}(15 \mathrm{~A})-\mathrm{C}(16 \mathrm{~A})-\mathrm{O}(2 \mathrm{~A})$ & $-57.53(13)$ \\
\hline$C(16 A)-O(2 A)-C(17 A)-C(18 A)$ & $-59.23(13)$ \\
\hline $\mathrm{C}(9 \mathrm{~A})-\mathrm{N}(2 \mathrm{~A})-\mathrm{C}(18 \mathrm{~A})-\mathrm{C}(17 \mathrm{~A})$ & $170.88(10)$ \\
\hline $\mathrm{C}(15 \mathrm{~A})-\mathrm{N}(2 \mathrm{~A})-\mathrm{C}(18 \mathrm{~A})-\mathrm{C}(17 \mathrm{~A})$ & $-57.83(13)$ \\
\hline $\mathrm{O}(2 \mathrm{~A})-\mathrm{C}(17 \mathrm{~A})-\mathrm{C}(18 \mathrm{~A})-\mathrm{N}(2 \mathrm{~A})$ & $59.85(13)$ \\
\hline
\end{tabular}


Symmetry transformations used to generate equivalent atoms: 
NMR Spectra 
<smiles>COc1ccc(CN(C(=O)c2ccccc2)c2ccccc2I)cc1</smiles>
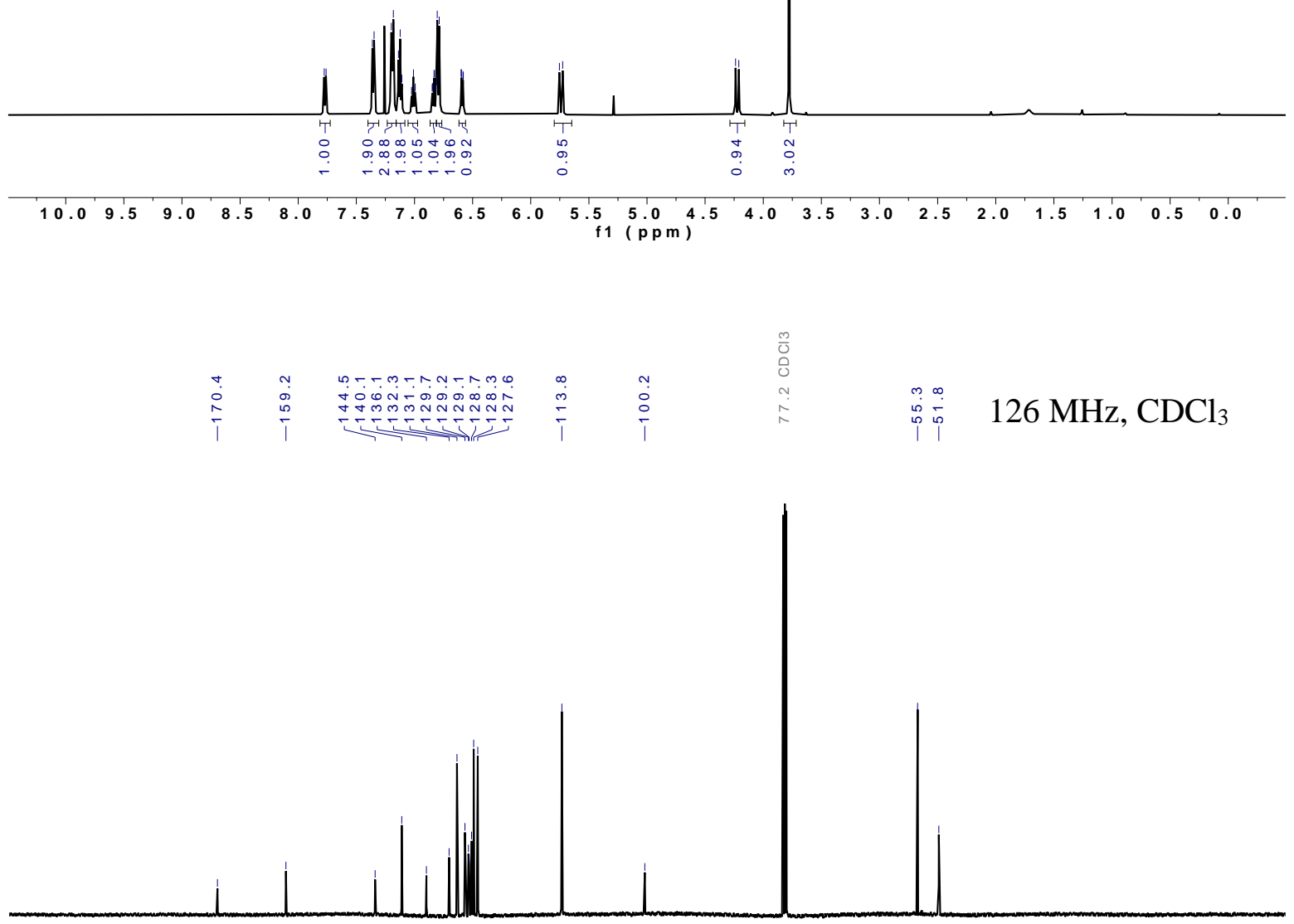

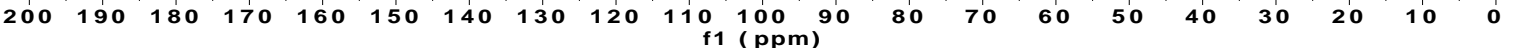



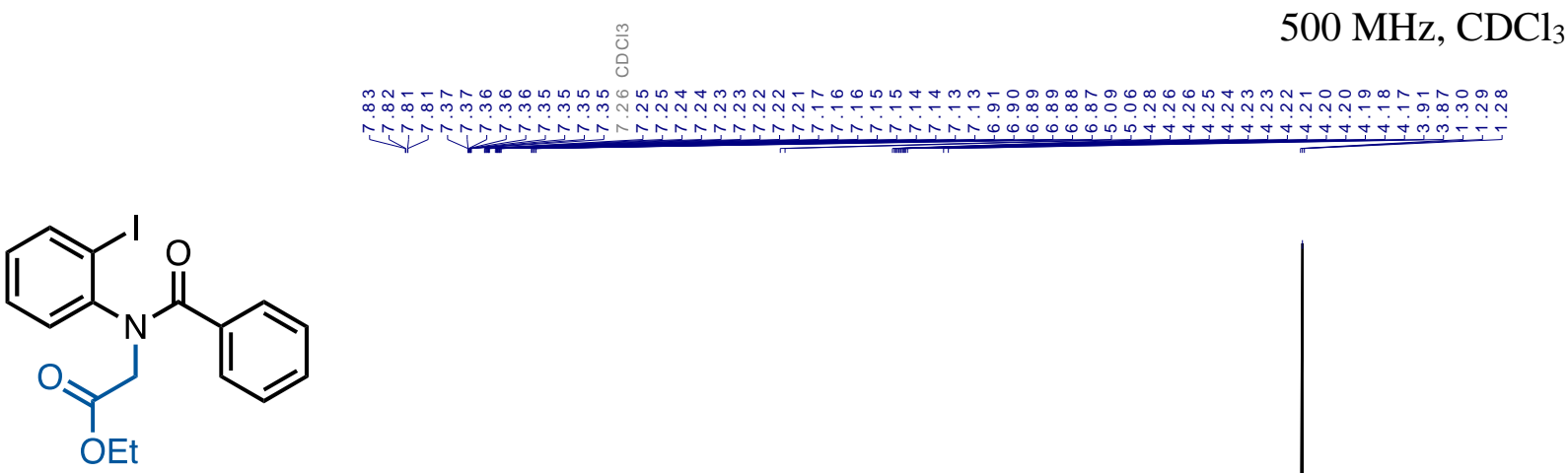

1d
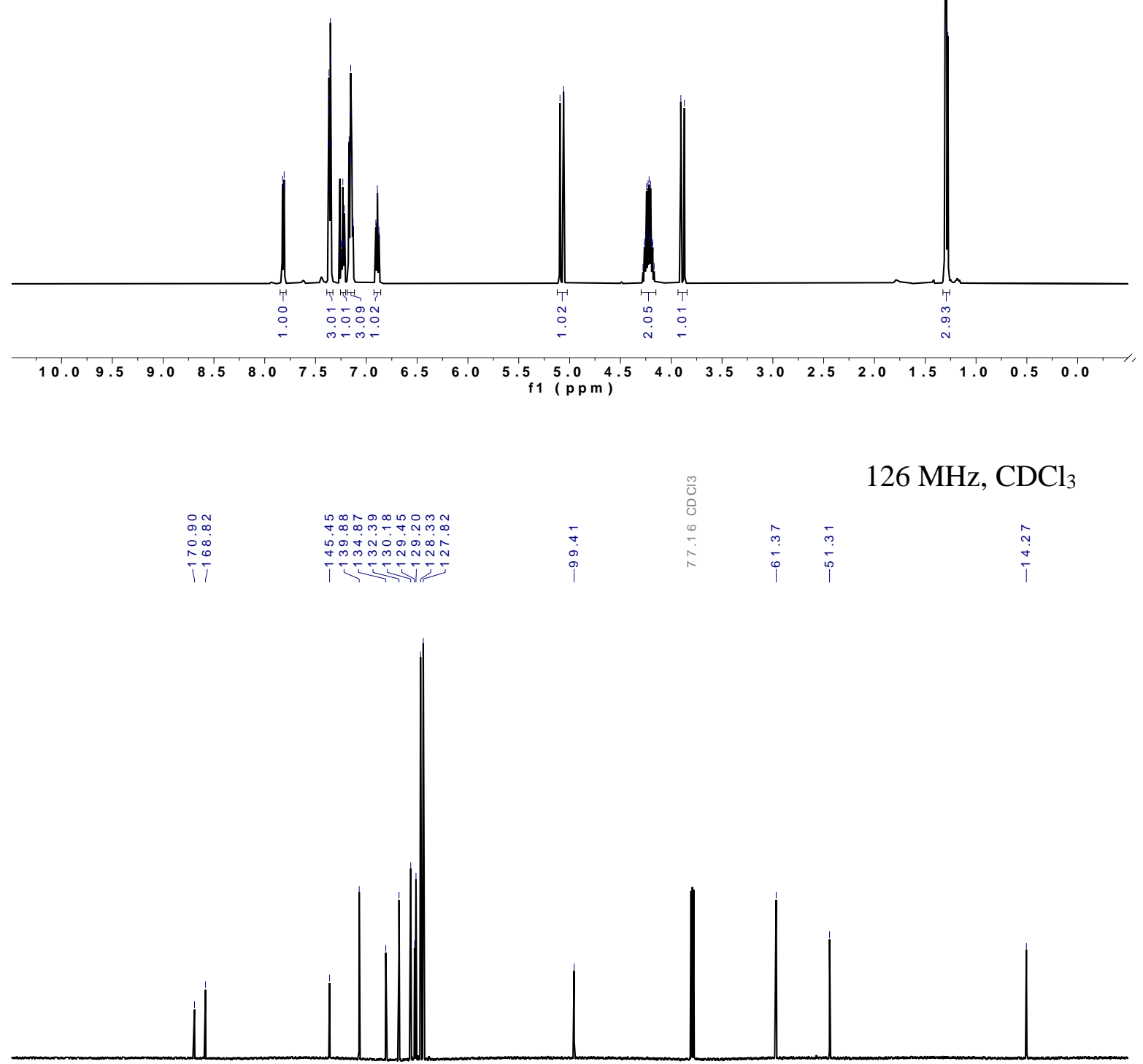

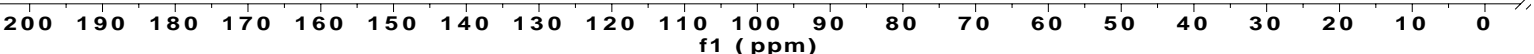



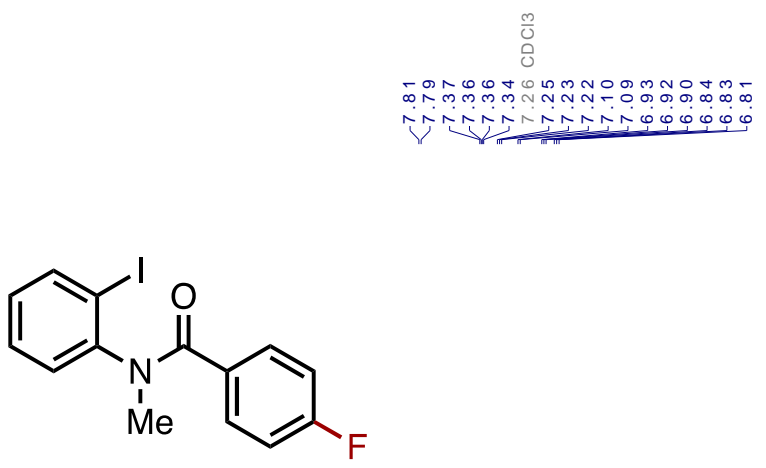

$1 i$
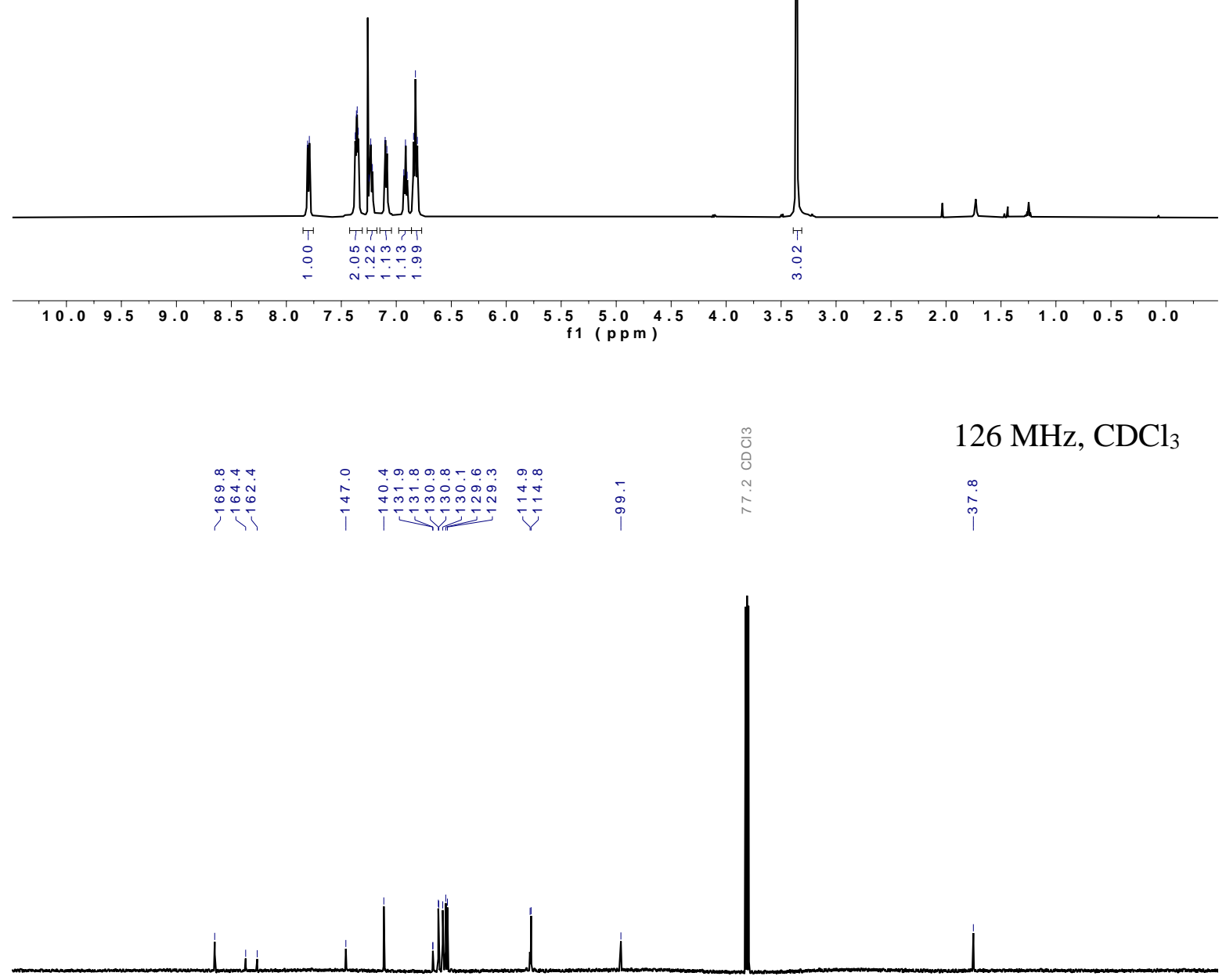

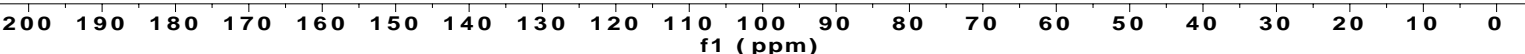




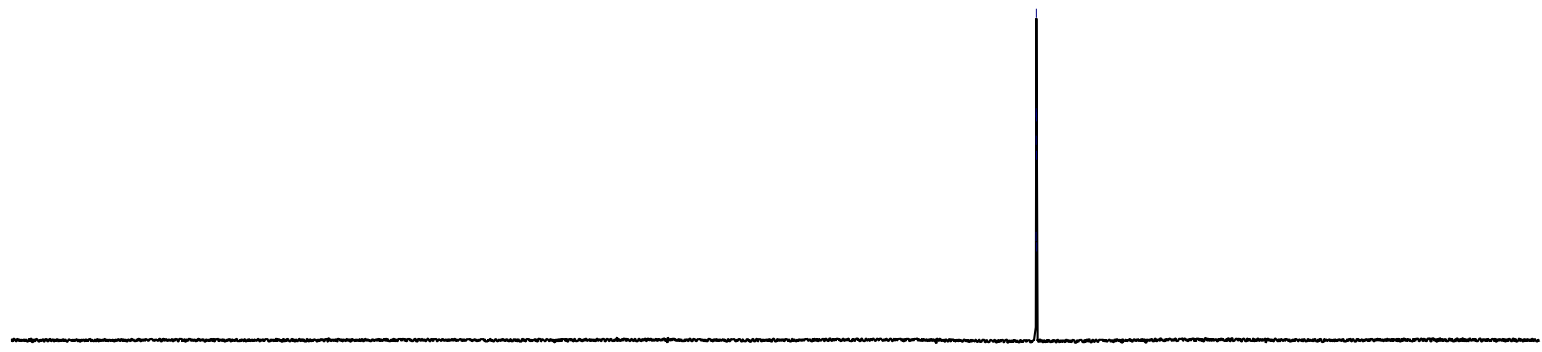

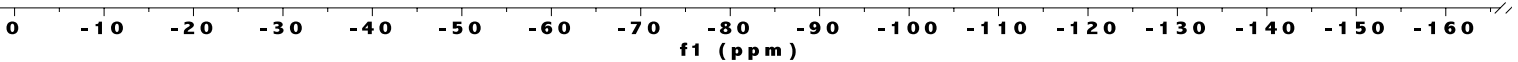



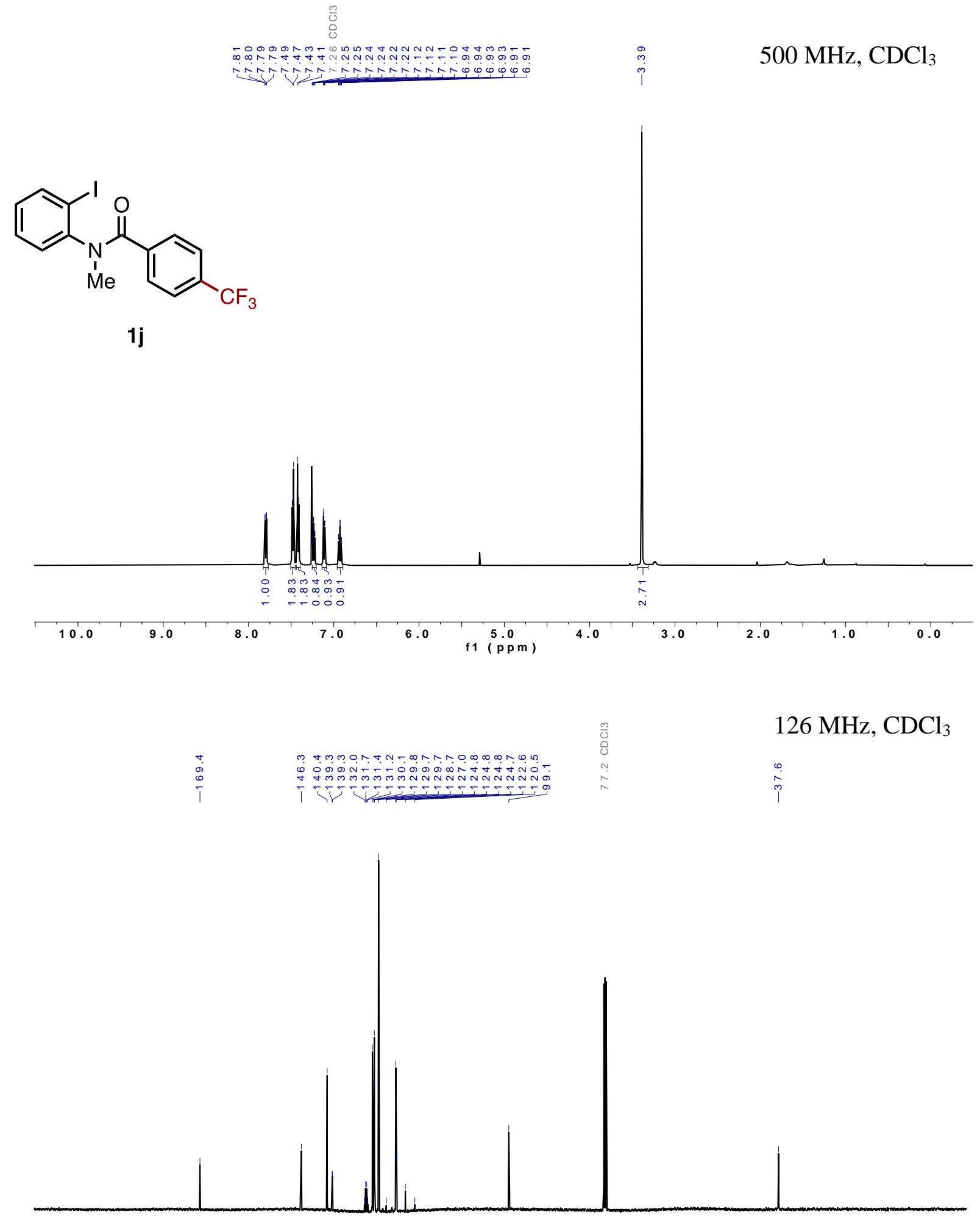

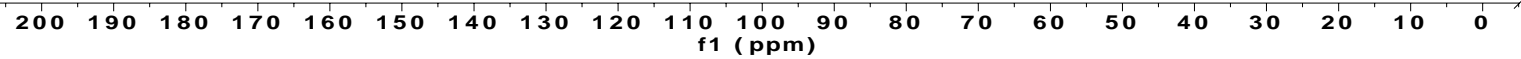




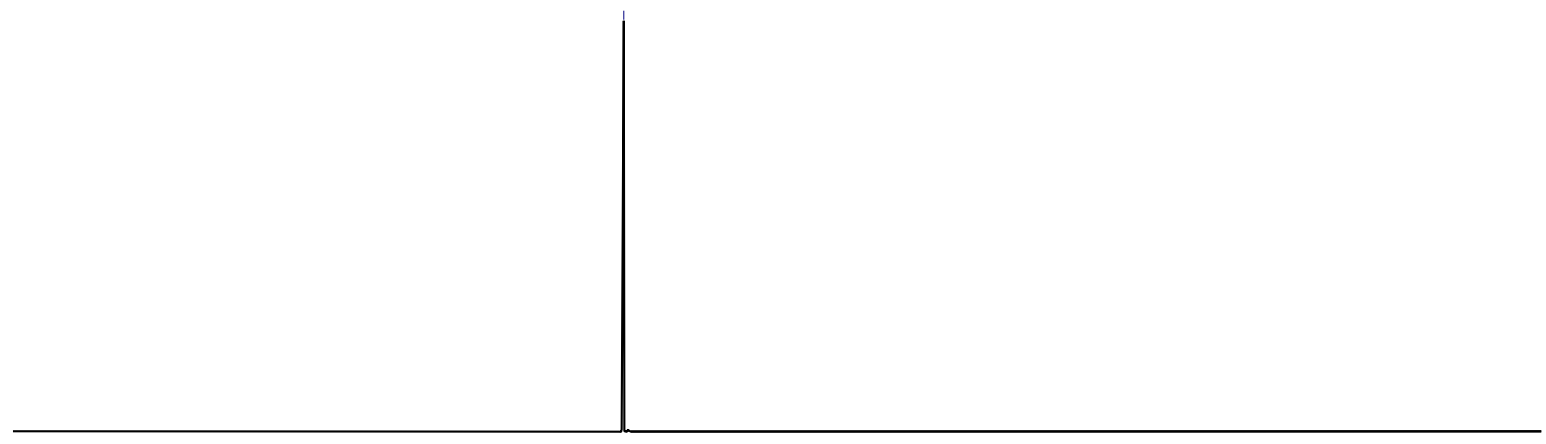

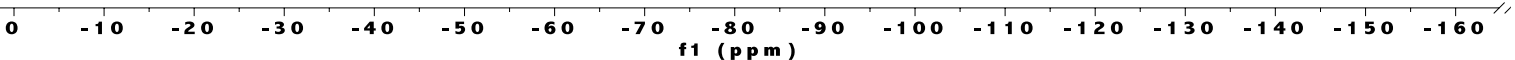



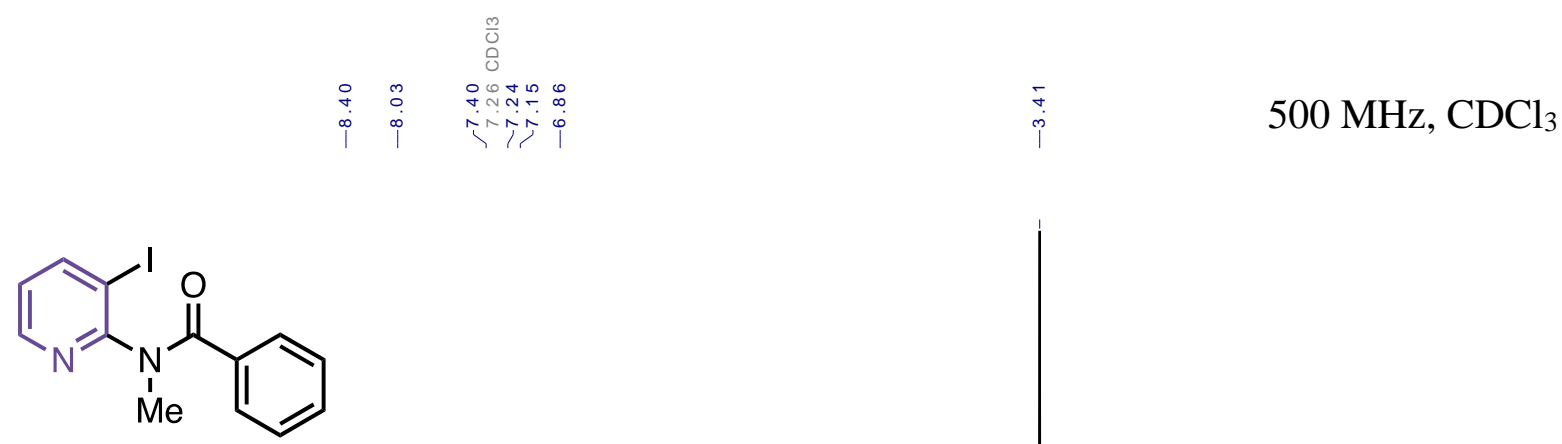

$1 \mathrm{~m}$

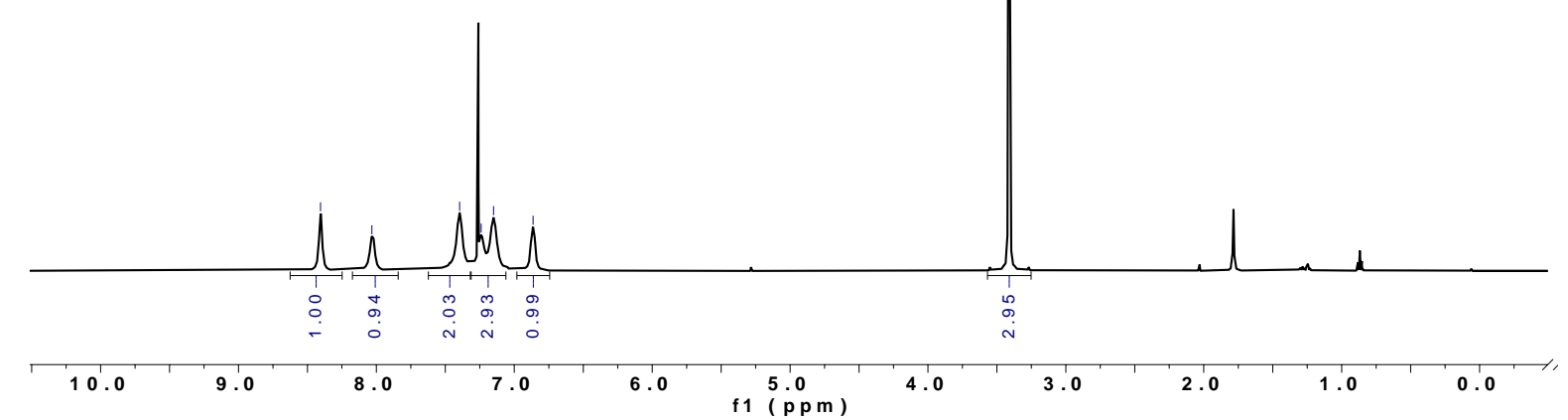

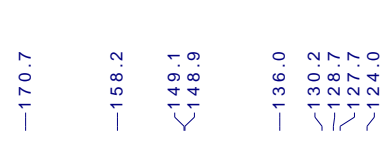

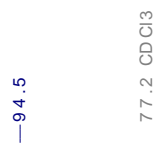

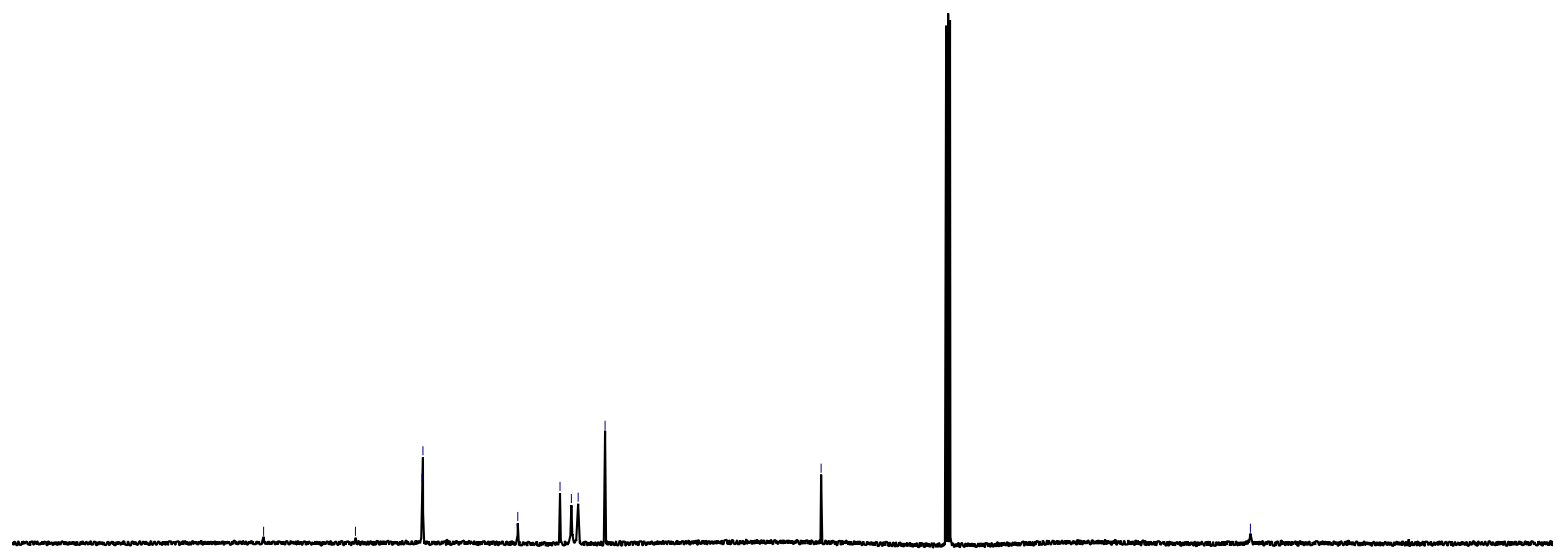

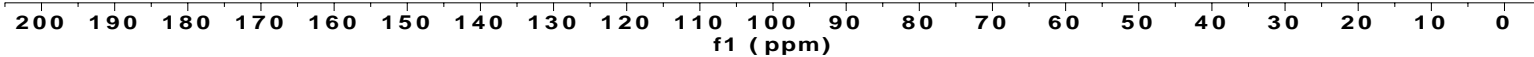




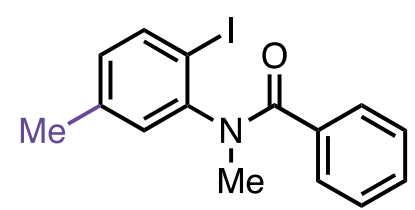

$1 n$
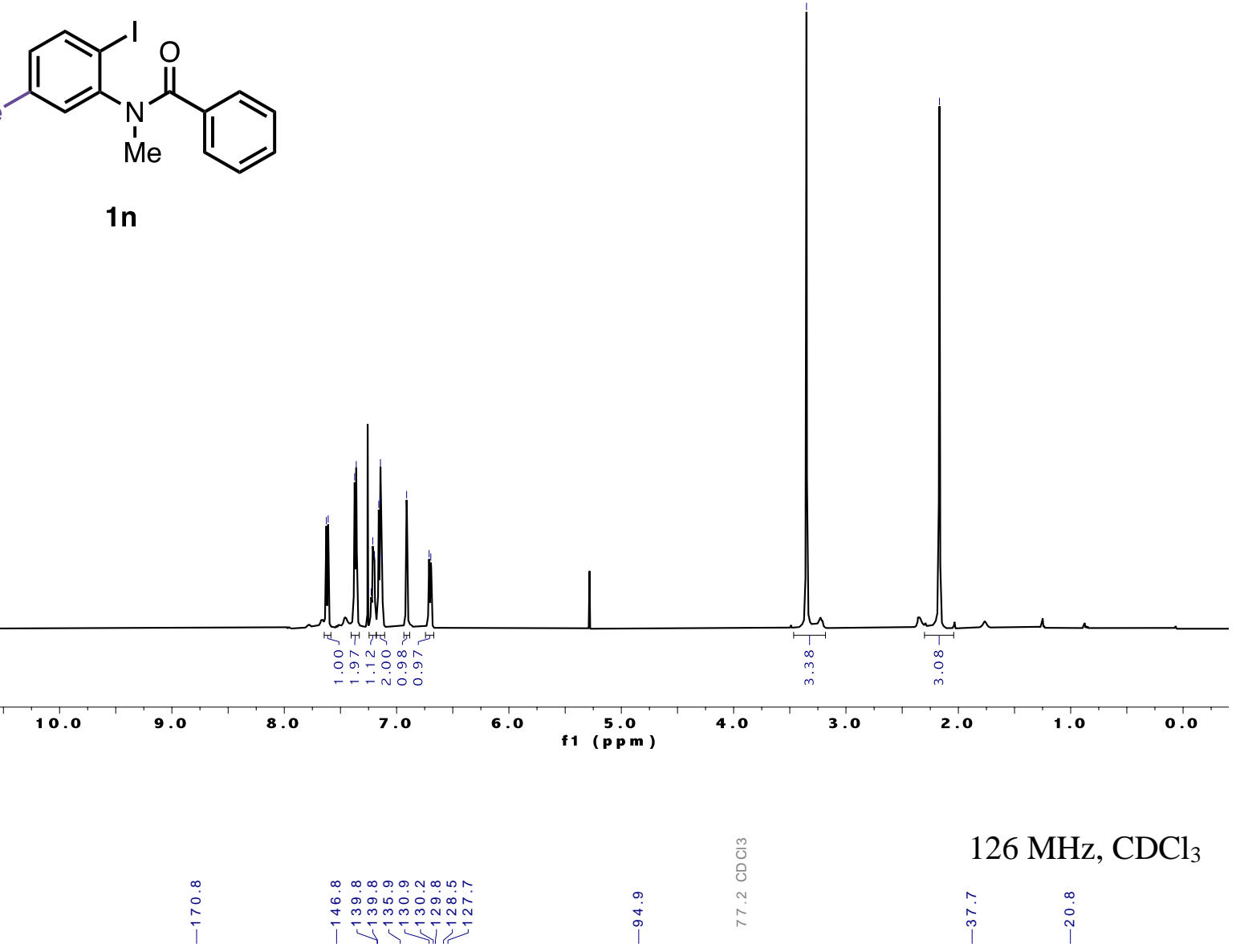

$126 \mathrm{MHz}, \mathrm{CDCl}_{3}$

$\frac{m}{0}$
0
0
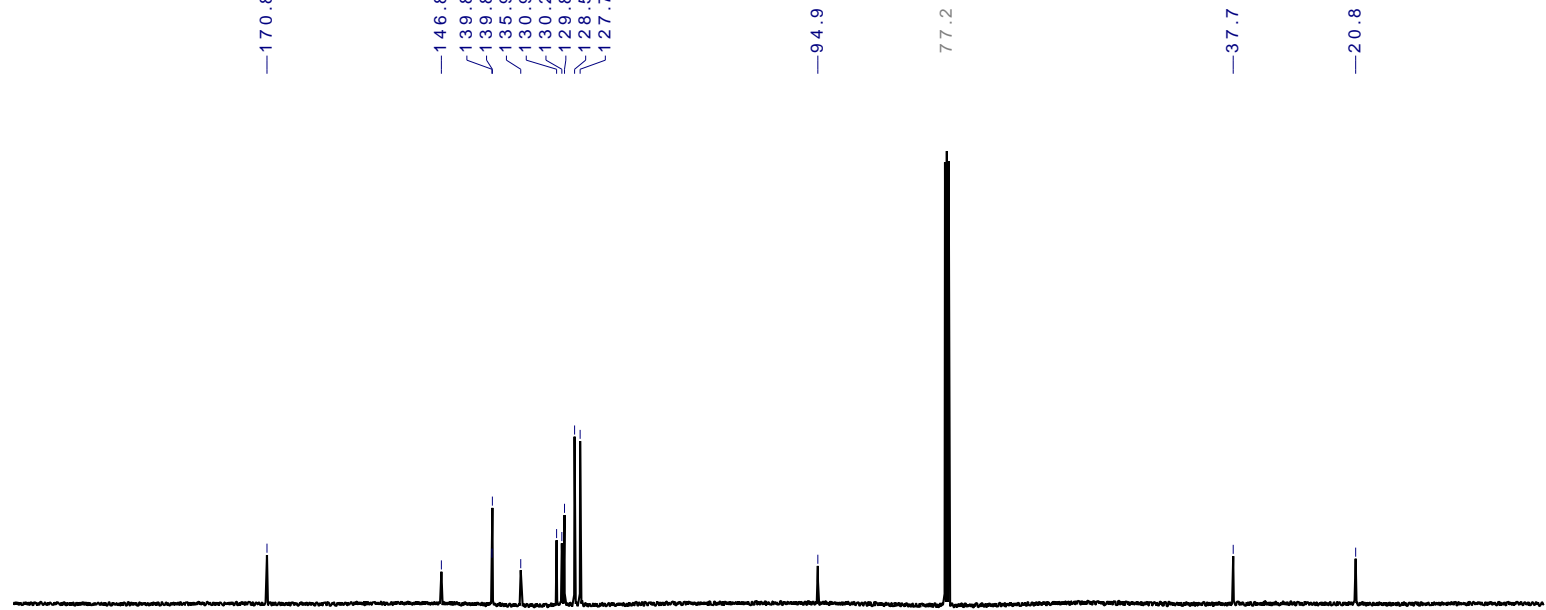

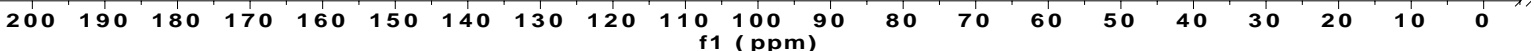




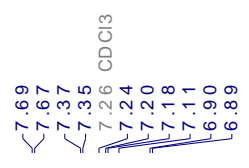<smiles>CN(C(=O)c1ccccc1)c1cc(Cl)ccc1I</smiles>
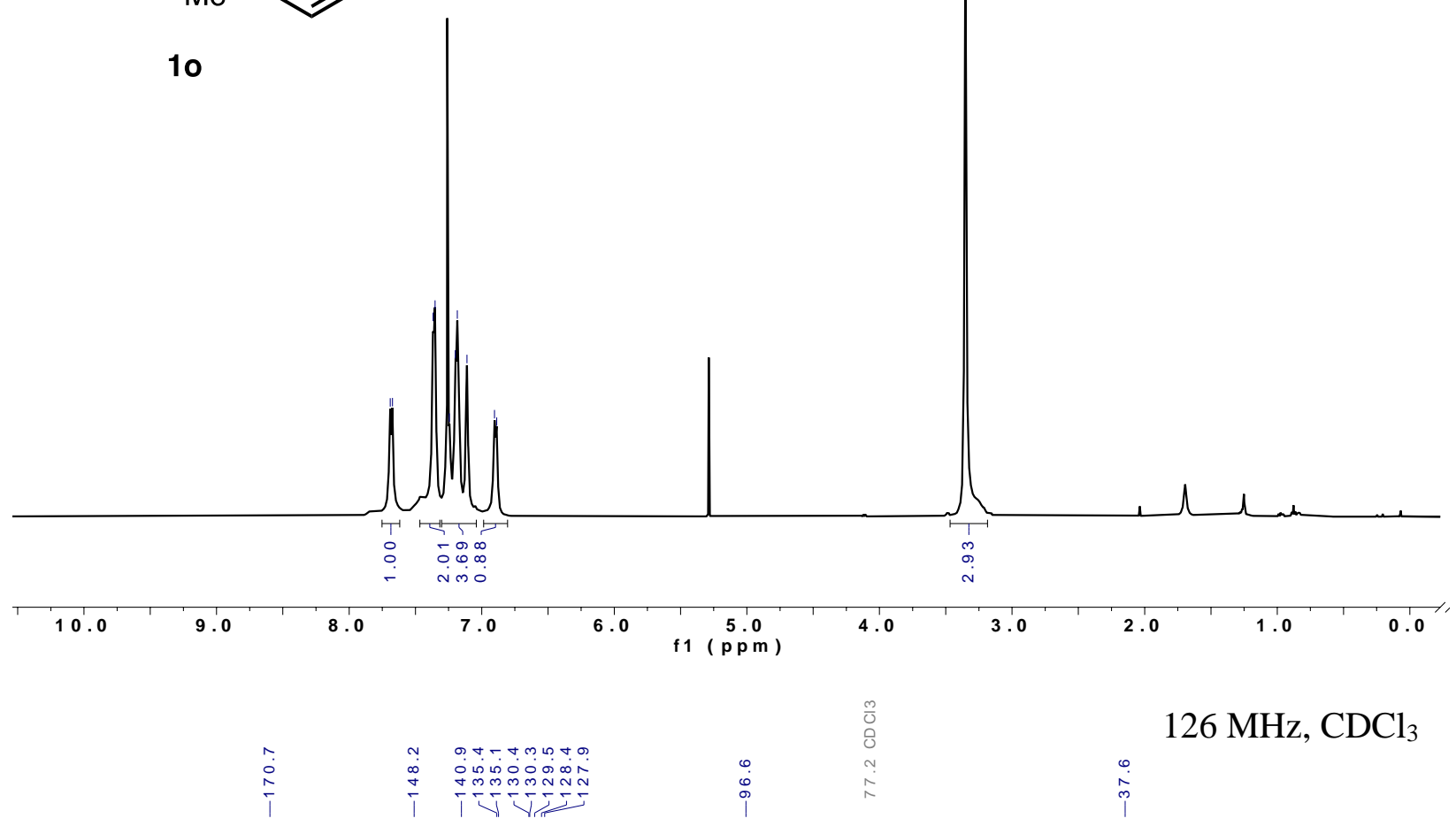

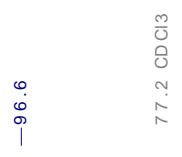

$126 \mathrm{MHz}, \mathrm{CDCl}_{3}$

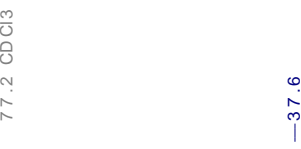

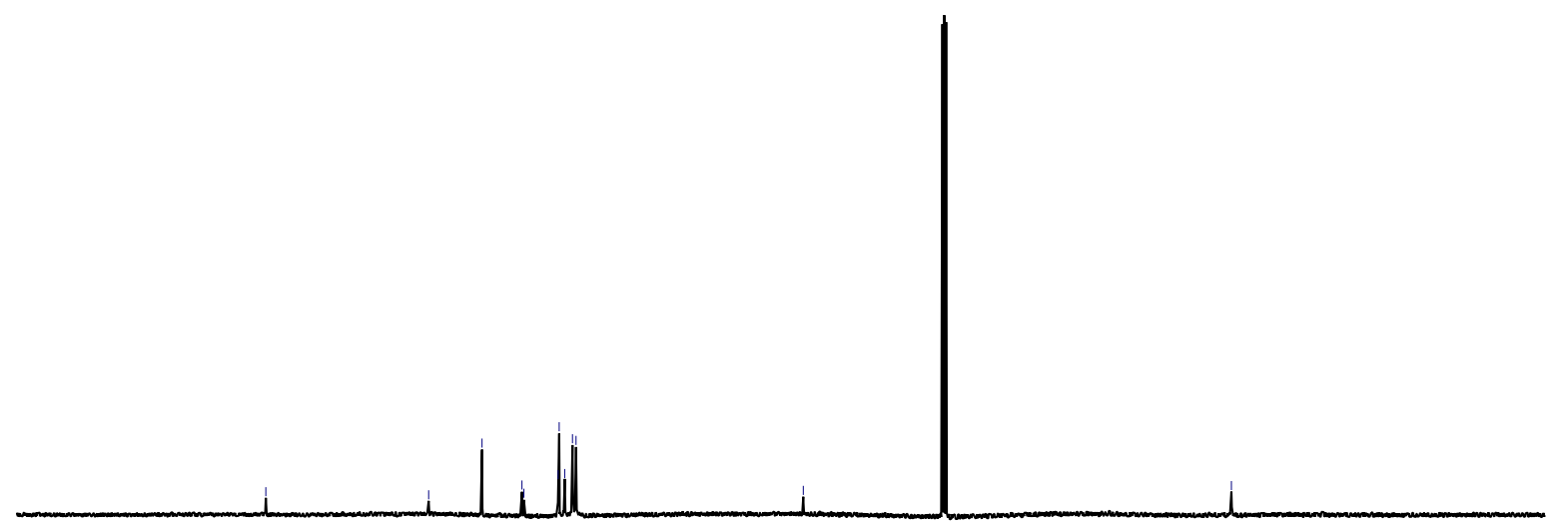

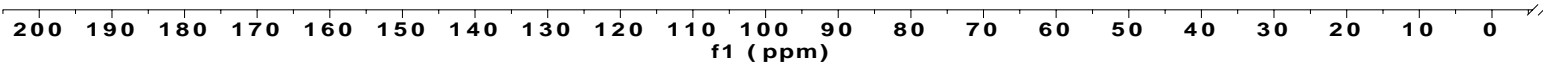



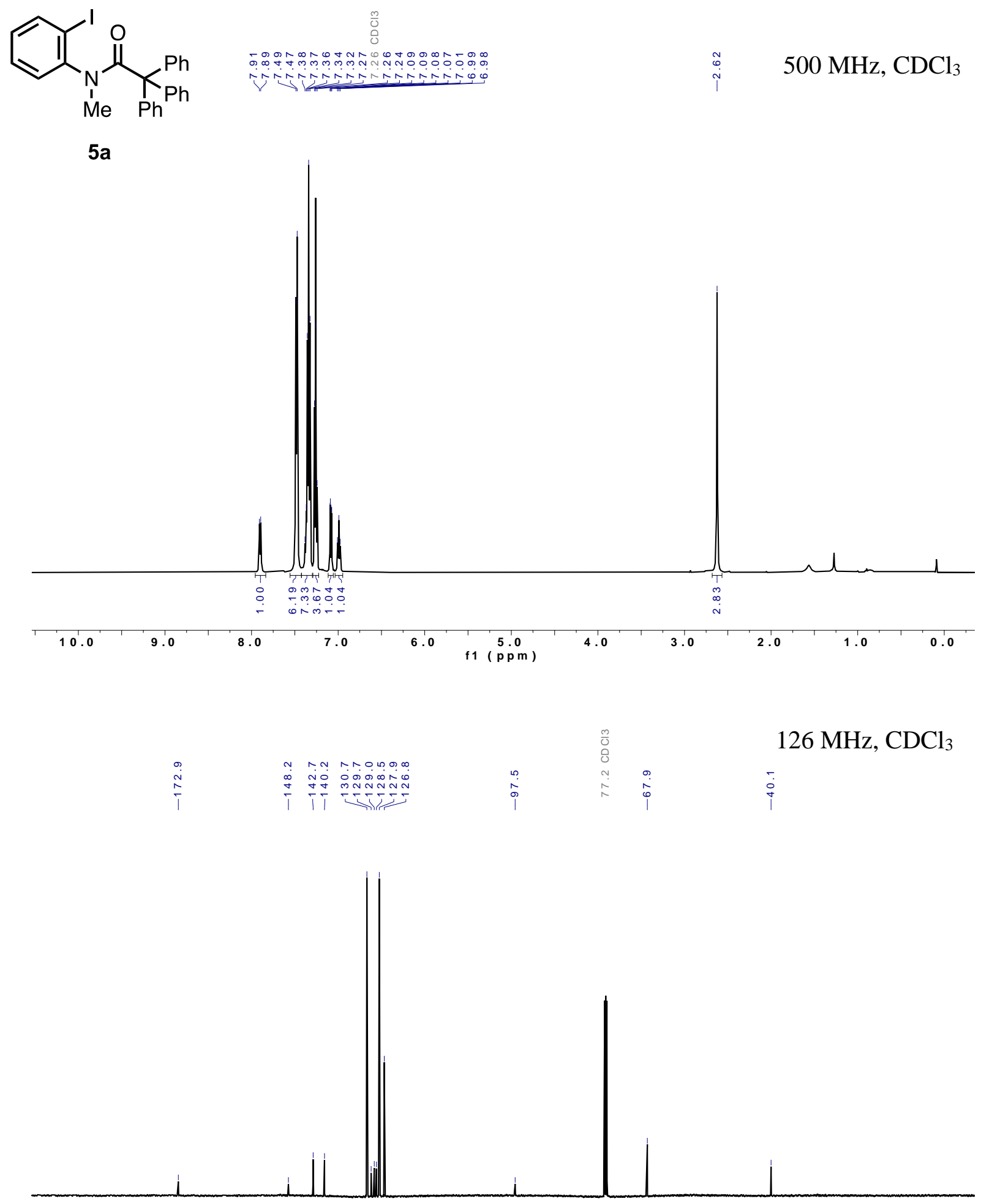

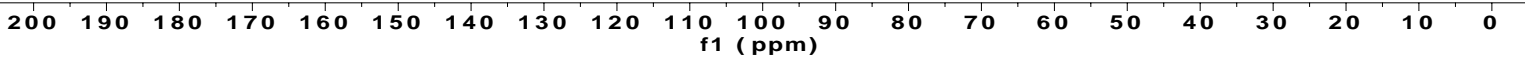



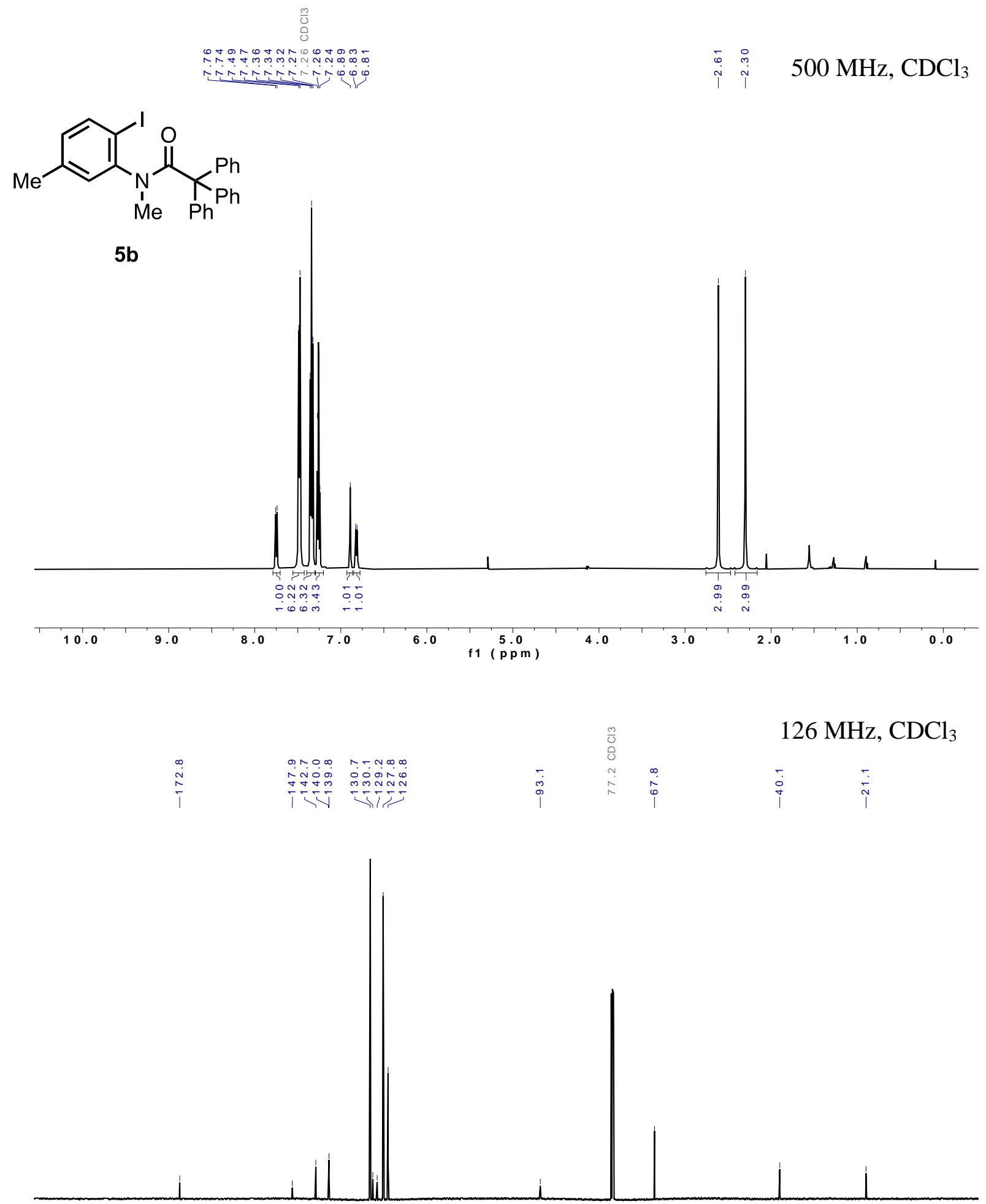

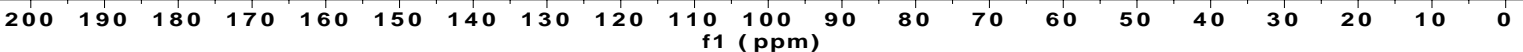



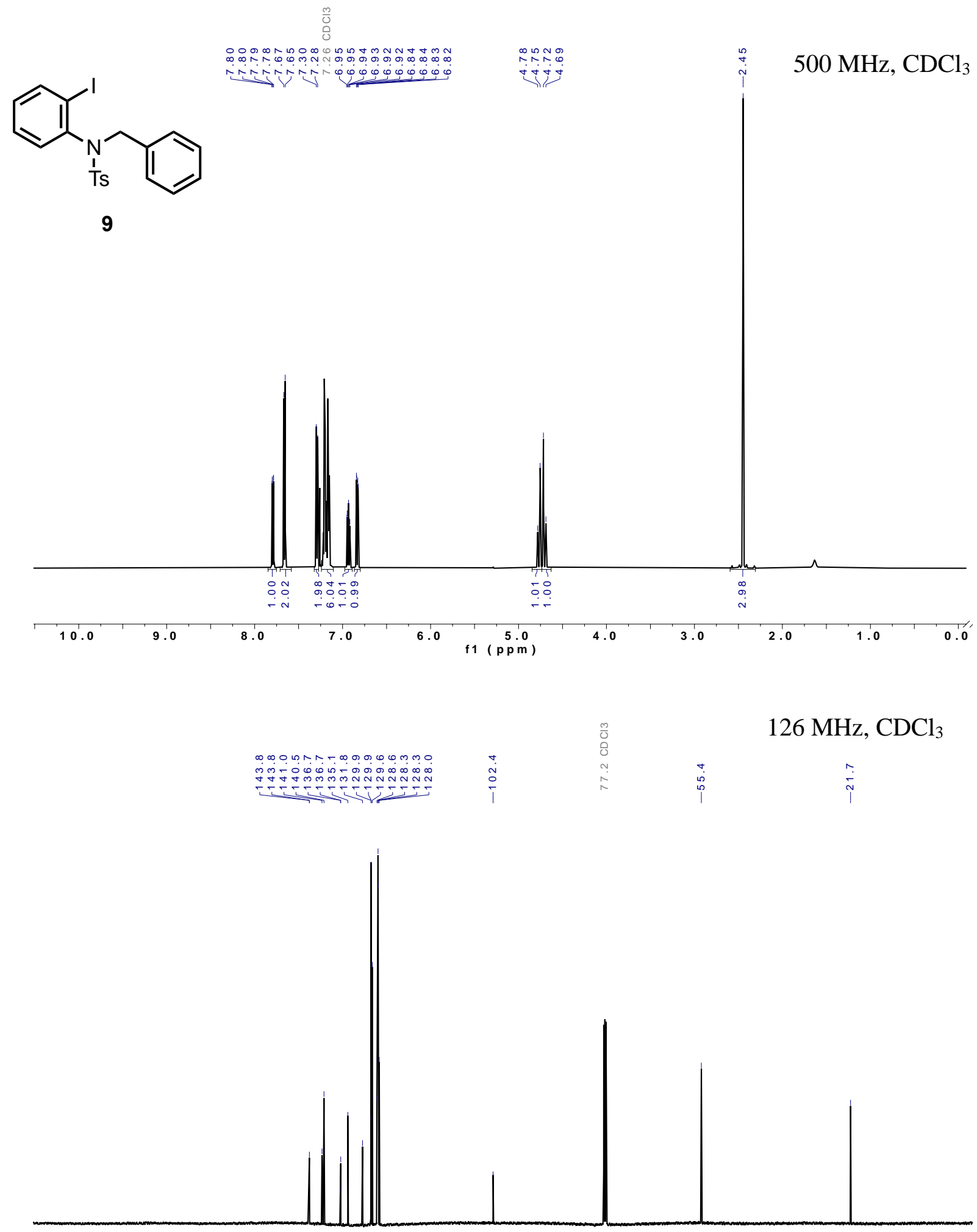

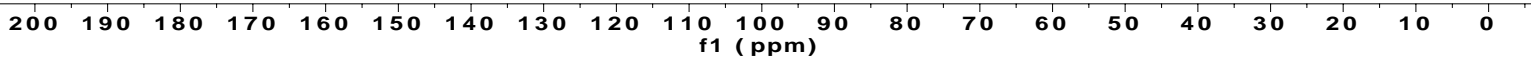



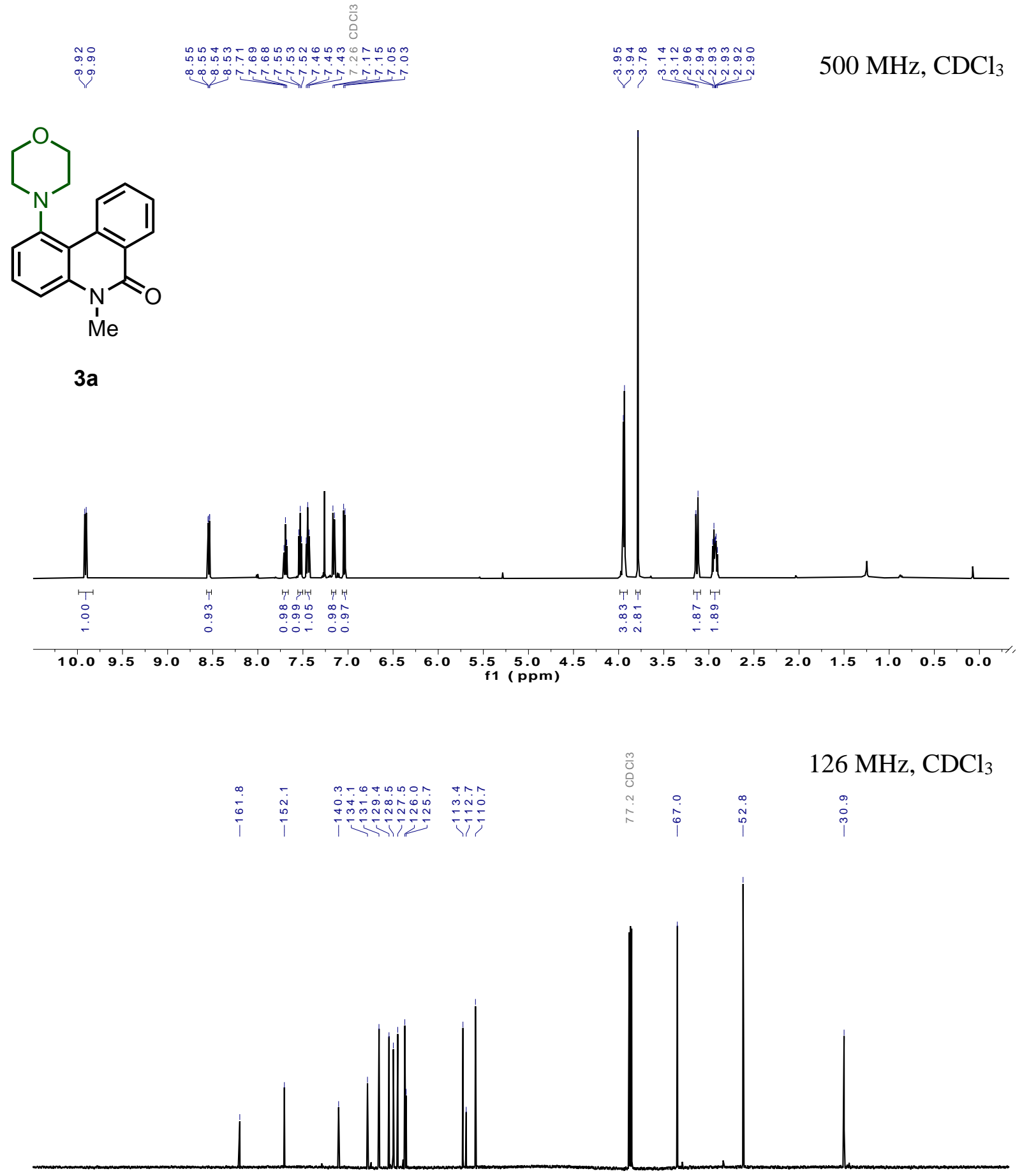

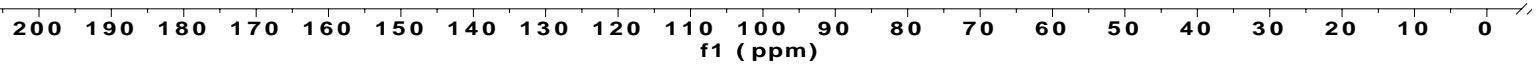


<smiles>O=c1c2ccccc2c2c(N3CCOCC3)cccc2n1Cc1ccccc1</smiles>

$3 b$

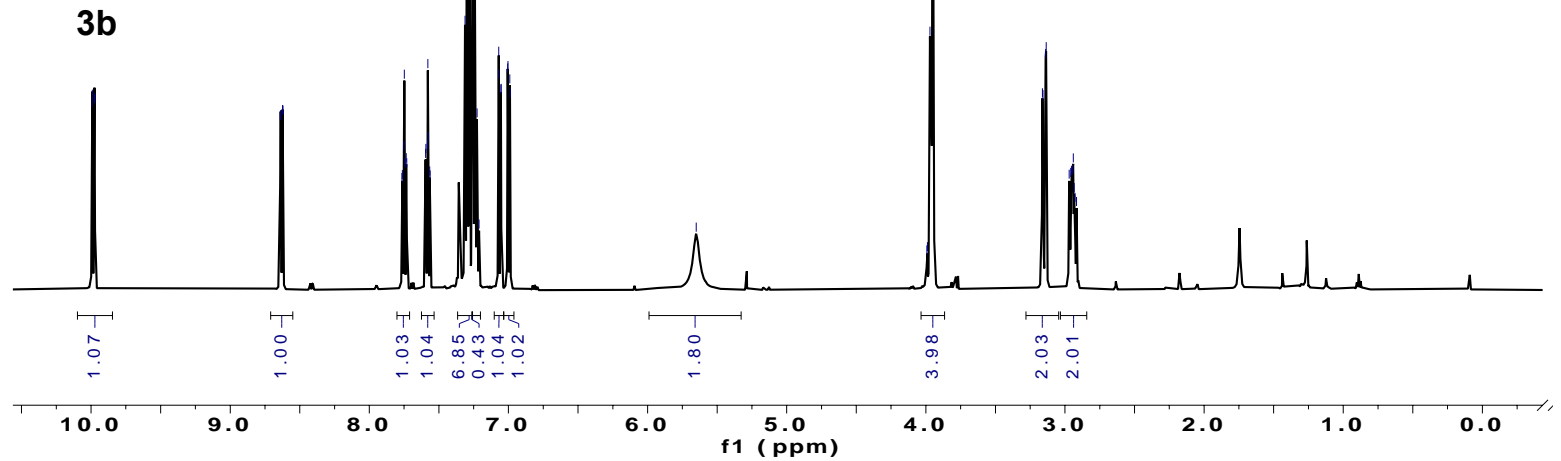

\begin{tabular}{lll}
1 & \multicolumn{1}{l}{$l$}
\end{tabular}
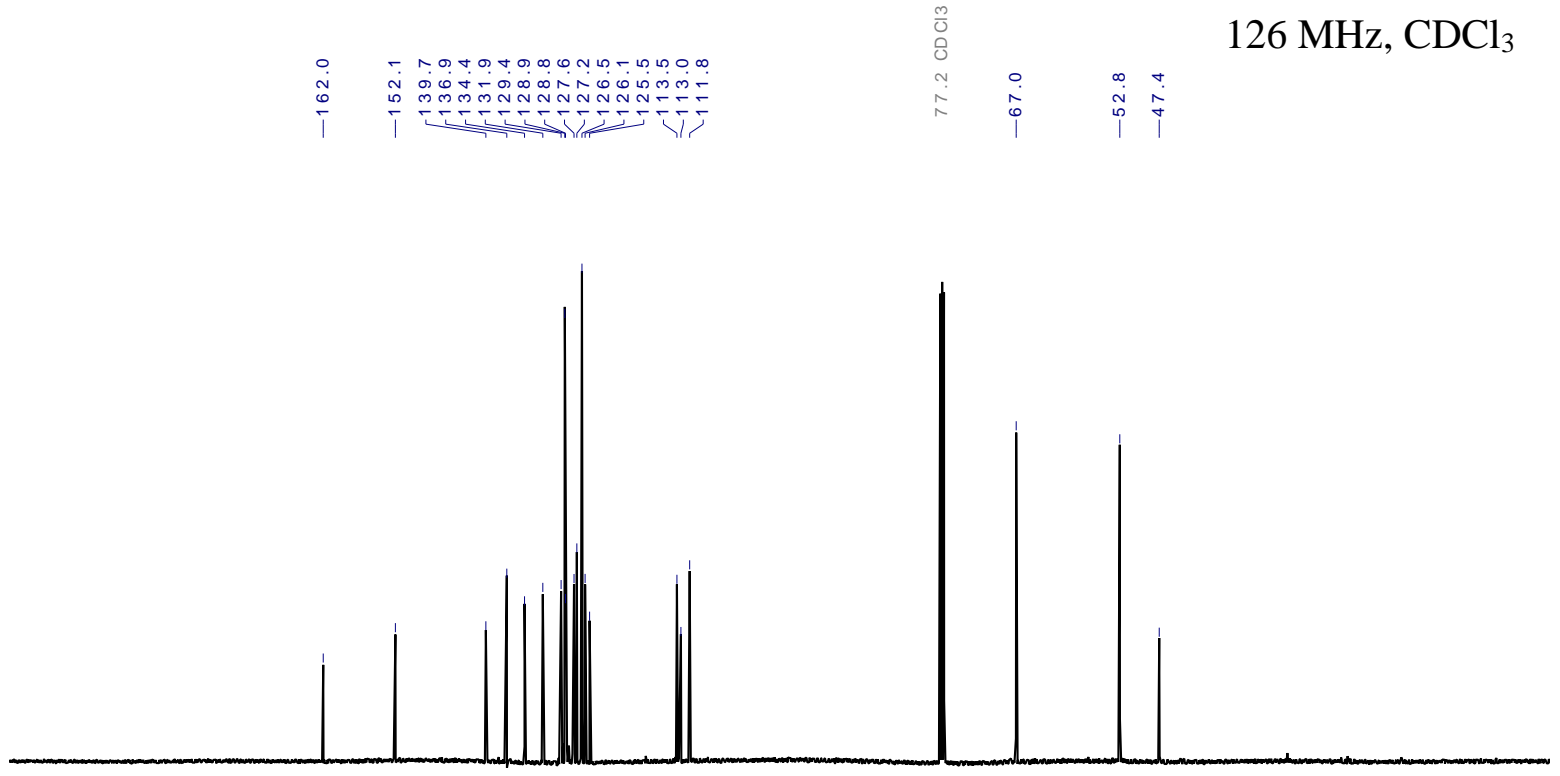

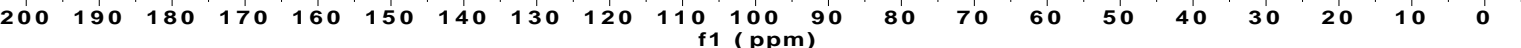


<smiles>COc1ccc(Cn2c(=O)c3ccccc3c3c(N4CCOCC4)cccc32)cc1</smiles>

$3 c$
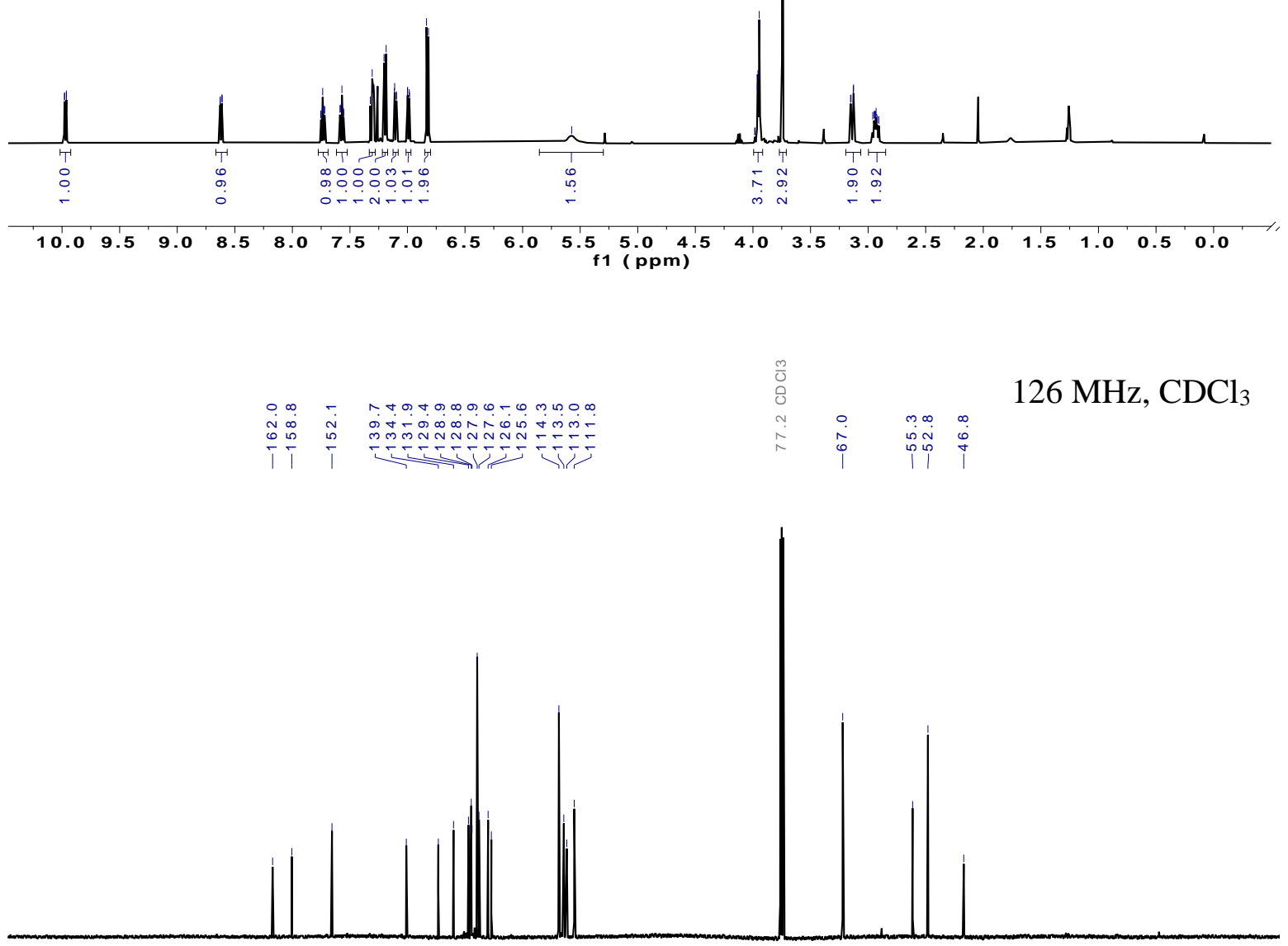

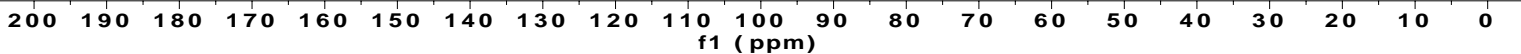


<smiles>CCOC(=O)Cn1c(=O)c2ccccc2c2c(N3CCOCC3)cccc21</smiles>

3d

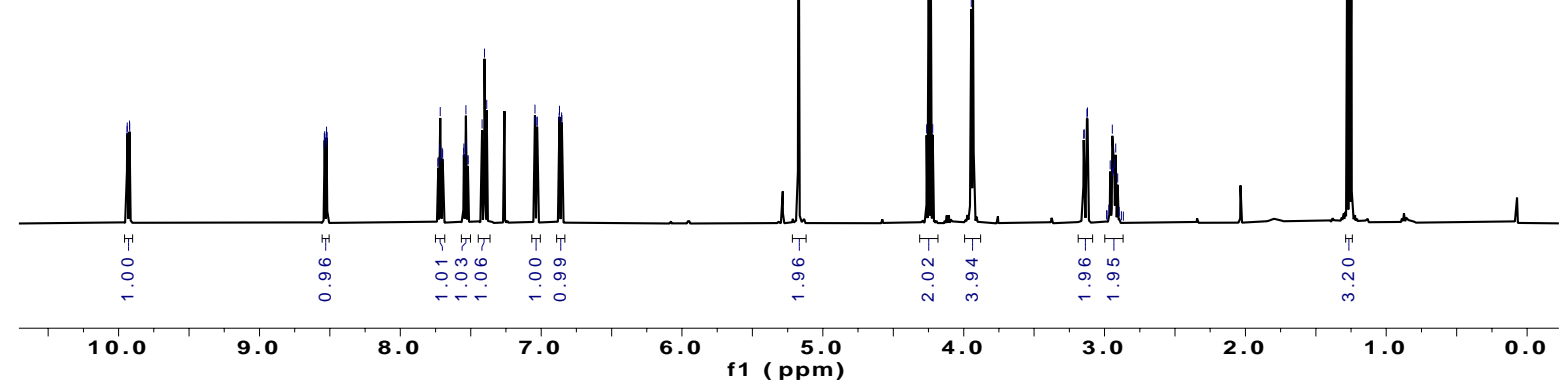


<smiles>Cc1ccc2c(=O)n(C)c3cccc(N4CCOCC4)c3c2c1</smiles>

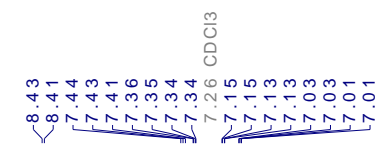

$500 \mathrm{MHz}, \mathrm{CDCl}_{3}$

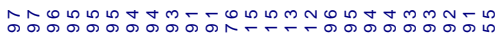

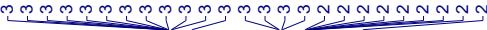

$3 e$

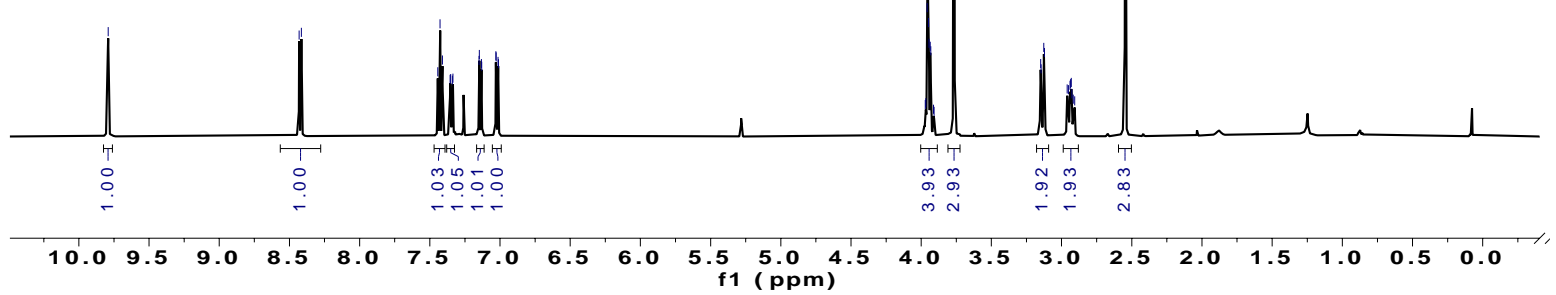

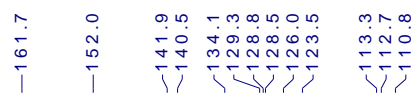
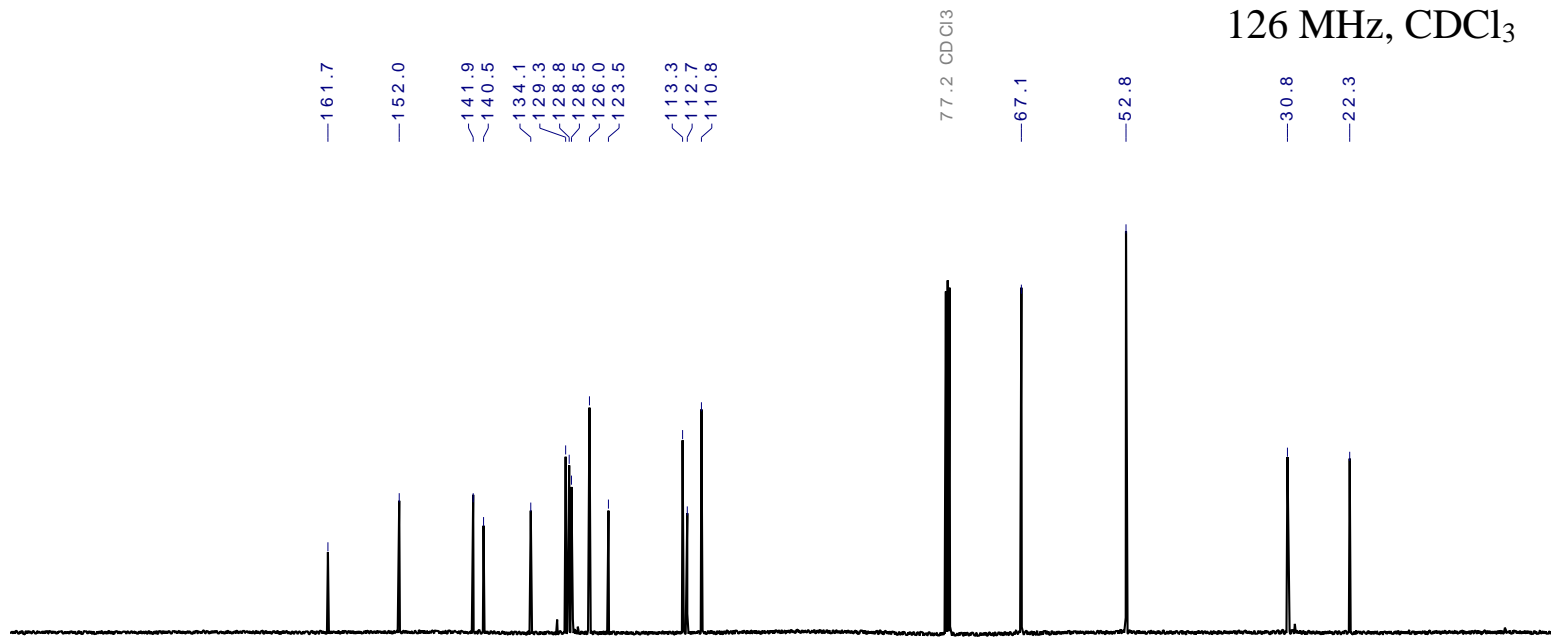

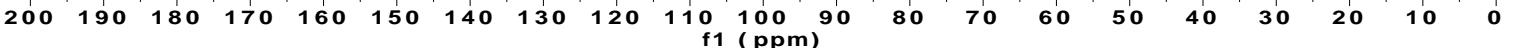




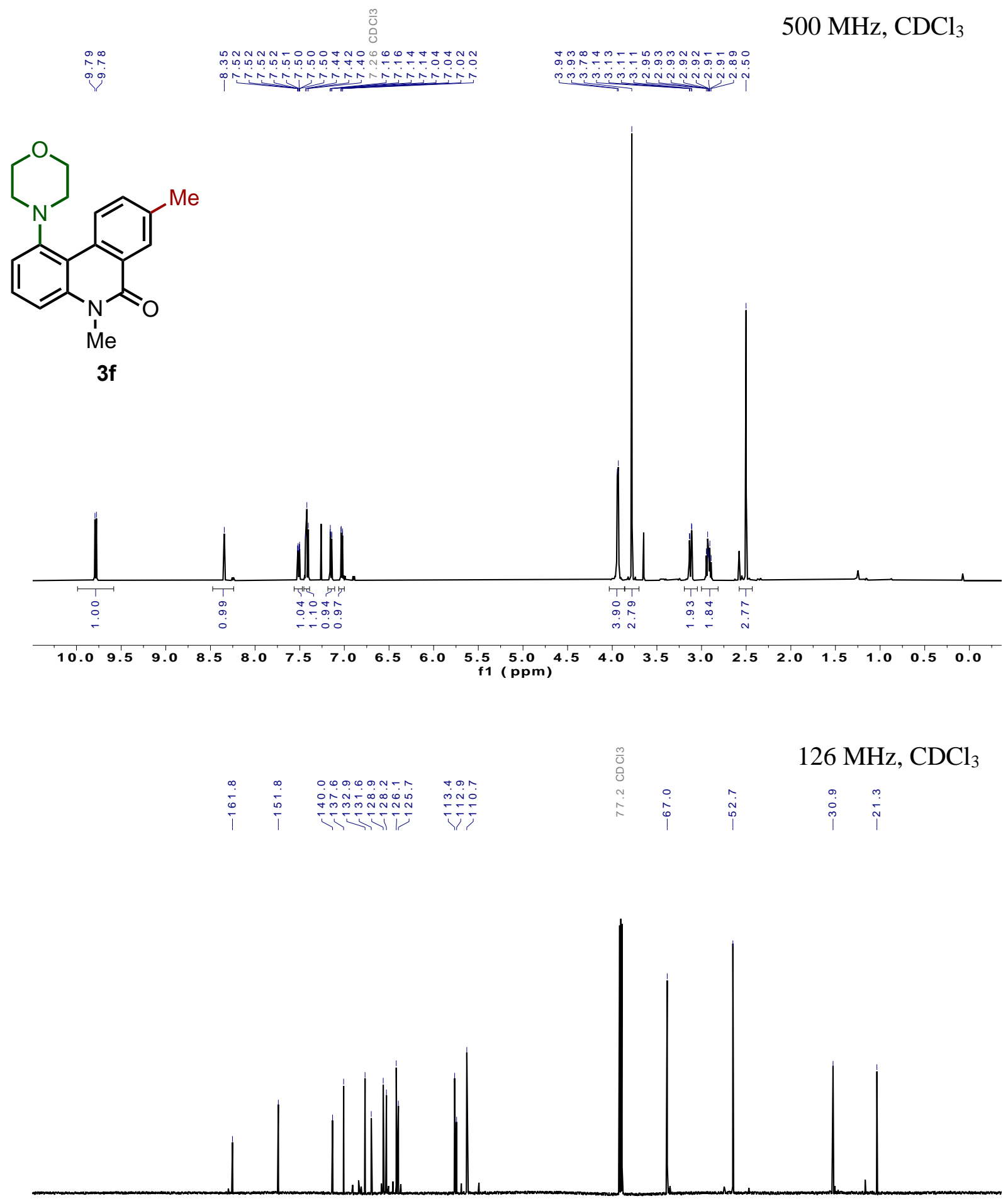

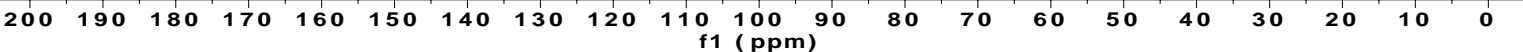



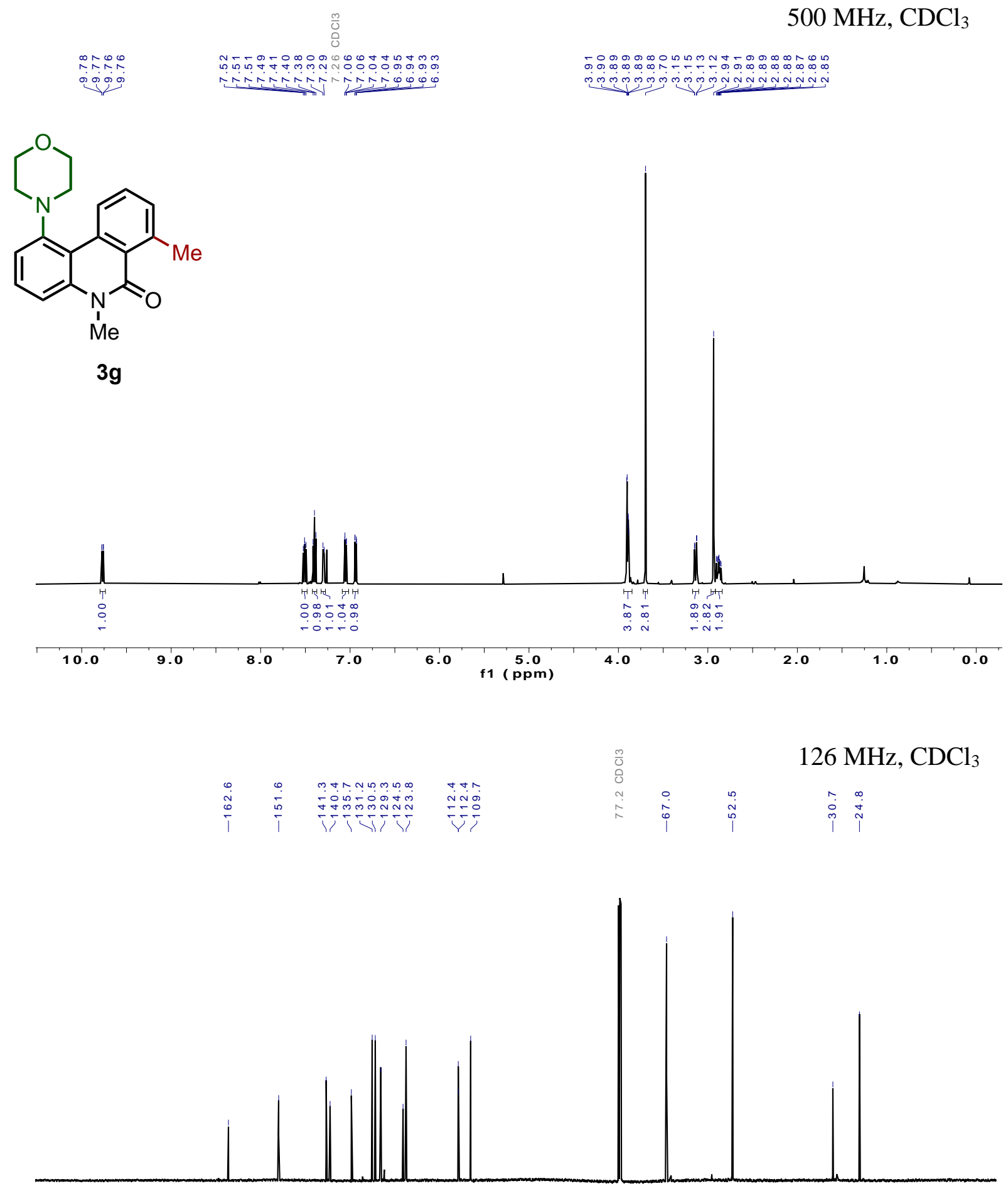

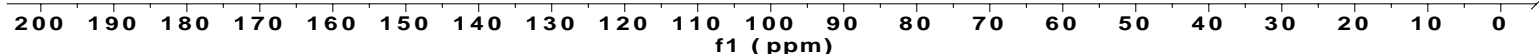


<smiles>COc1ccc2c(=O)n(C)c3cccc(N4CCOCC4)c3c2c1</smiles>

3h
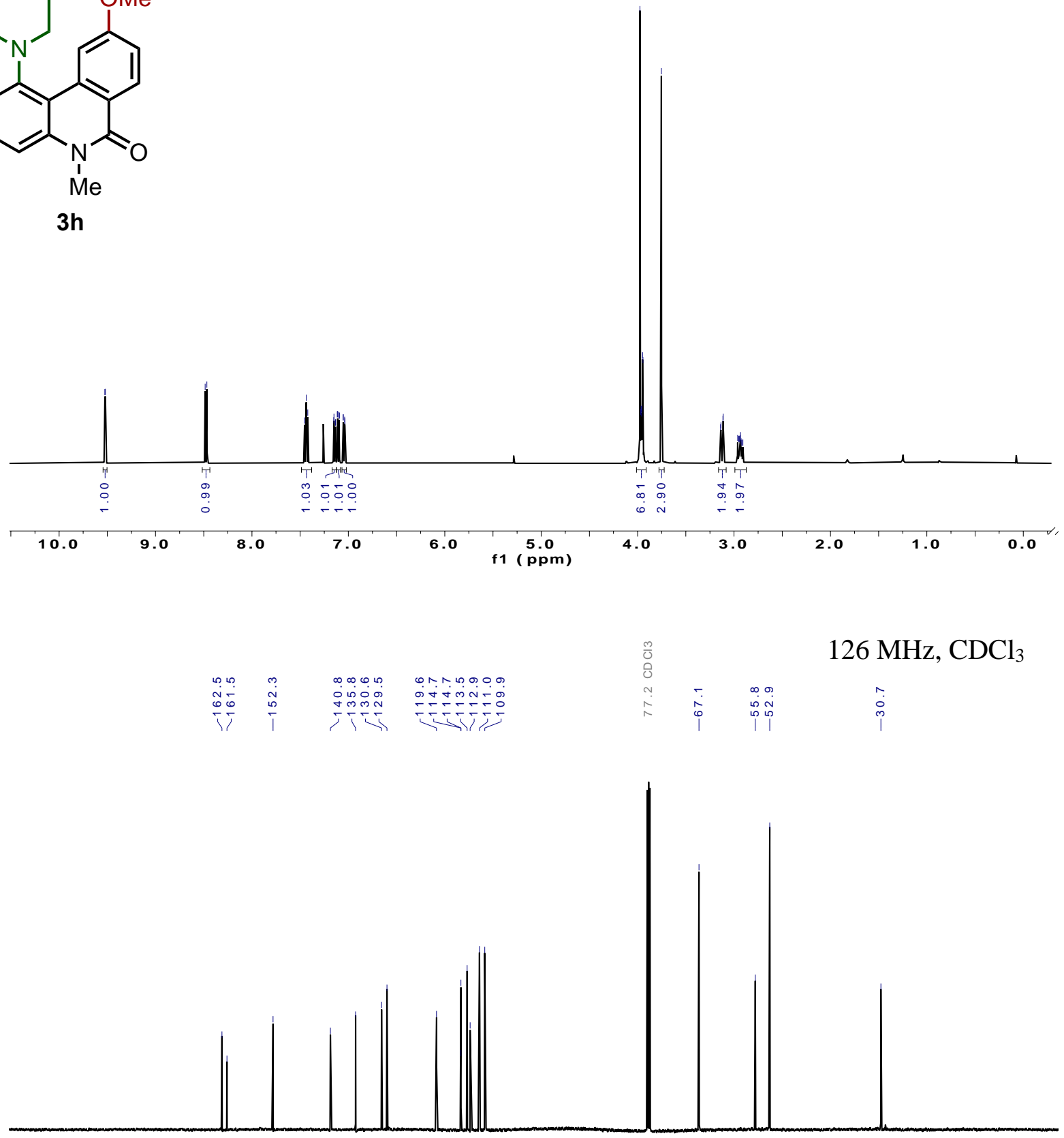

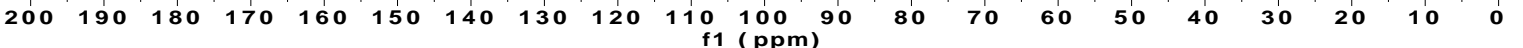




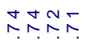

igio

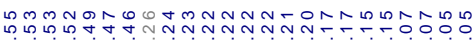

$\underbrace{\infty}_{0,0}$

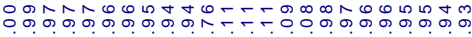

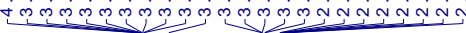<smiles>Cn1c(=O)c2ccc(F)cc2c2c(N3CCOCC3)cccc21</smiles>

$3 \mathbf{i}$

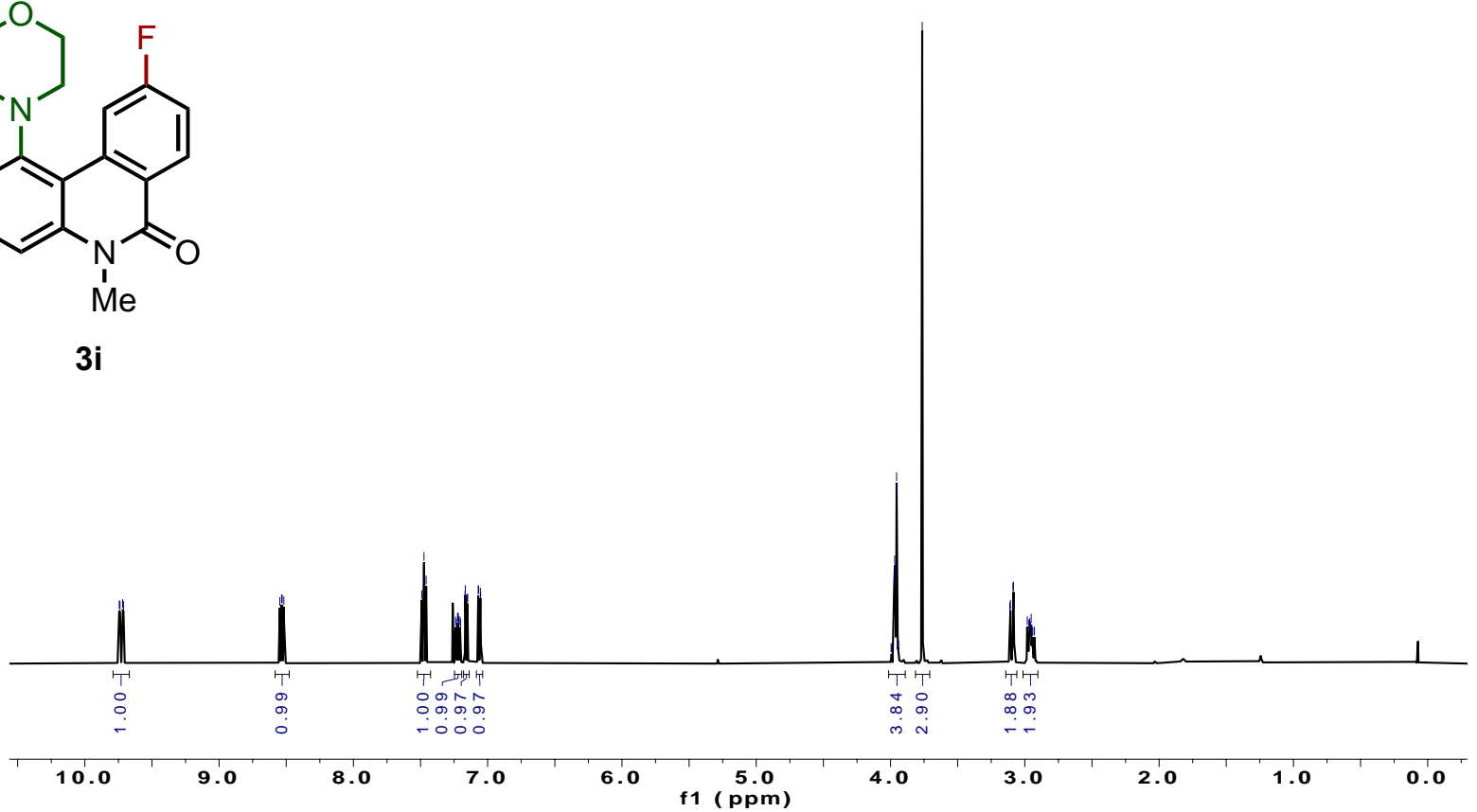

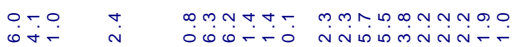

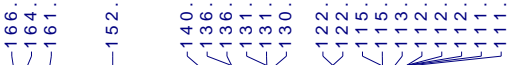

$\begin{array}{cccc}\frac{m}{0} & & & 126 \\ 0 & & & 126 \\ 0 & 0 & 0 & 0 \\ \uparrow & \dot{0} & \dot{0} & 0 \\ \wedge & 0 & 0 & 0\end{array}$

$126 \mathrm{MHz}, \mathrm{CDCl}_{3}$

111 । ये।

$\stackrel{9}{i}$

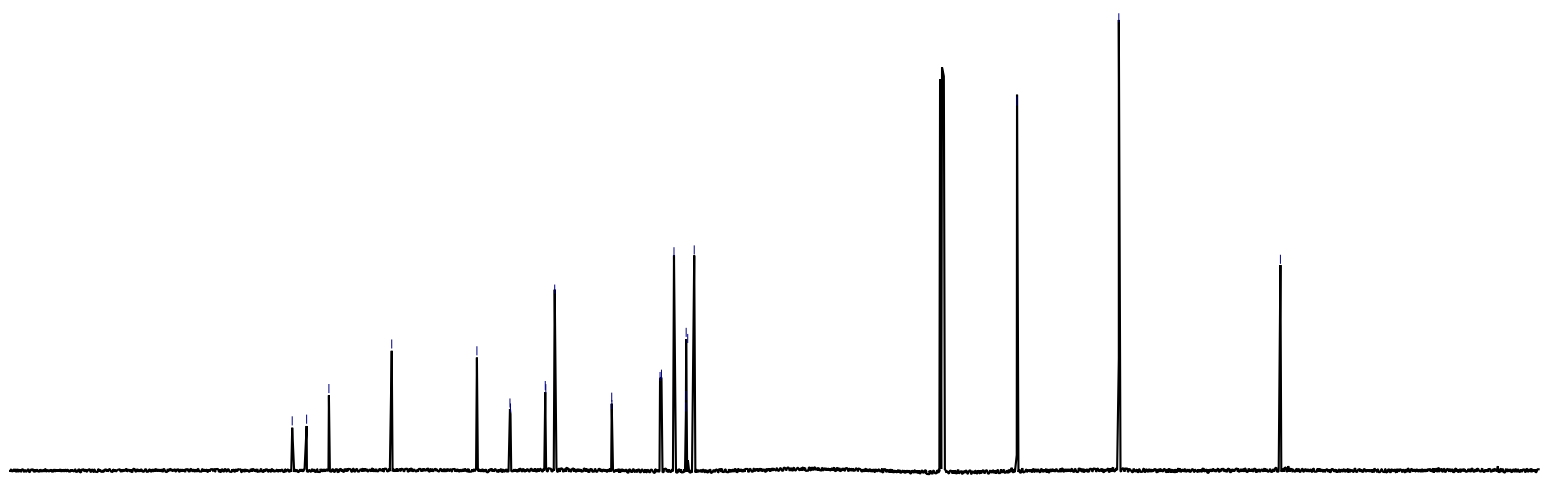

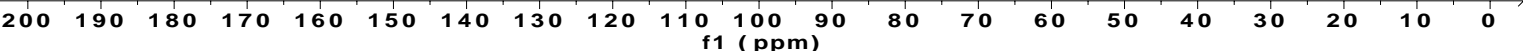




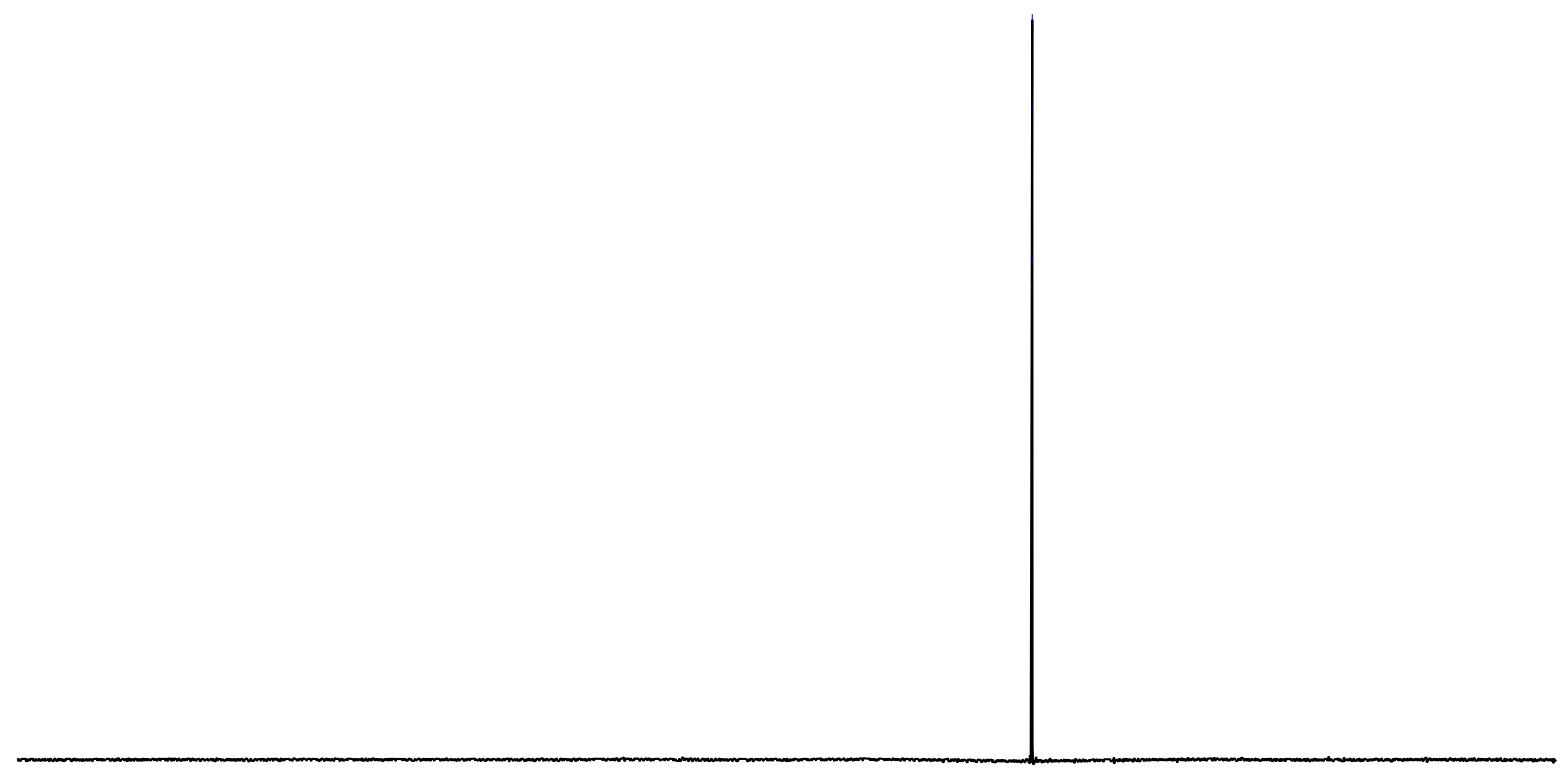




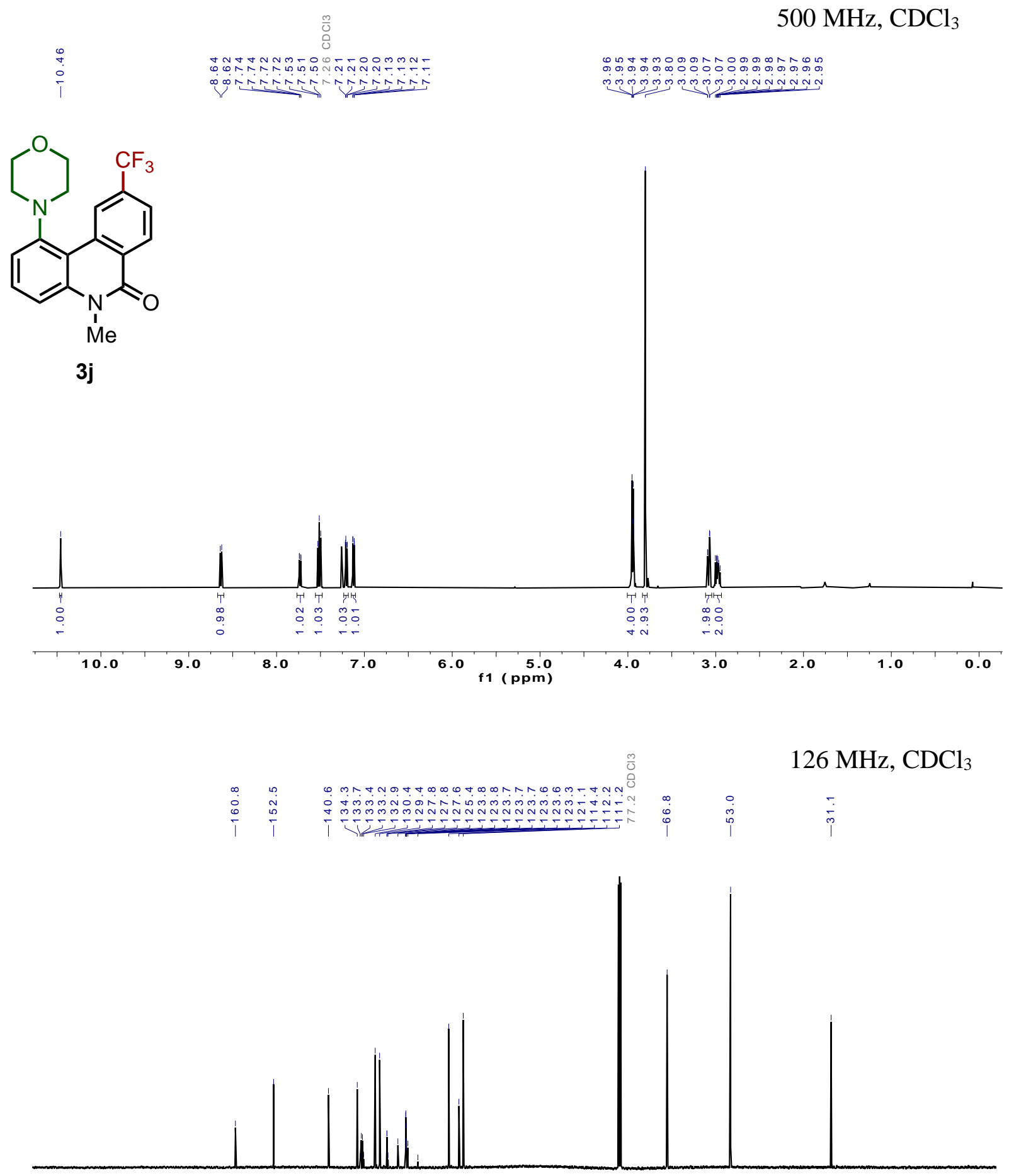

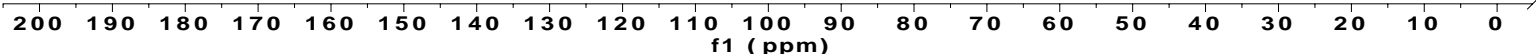


$375 \mathrm{MHz}, \mathrm{CDCl}_{3}$

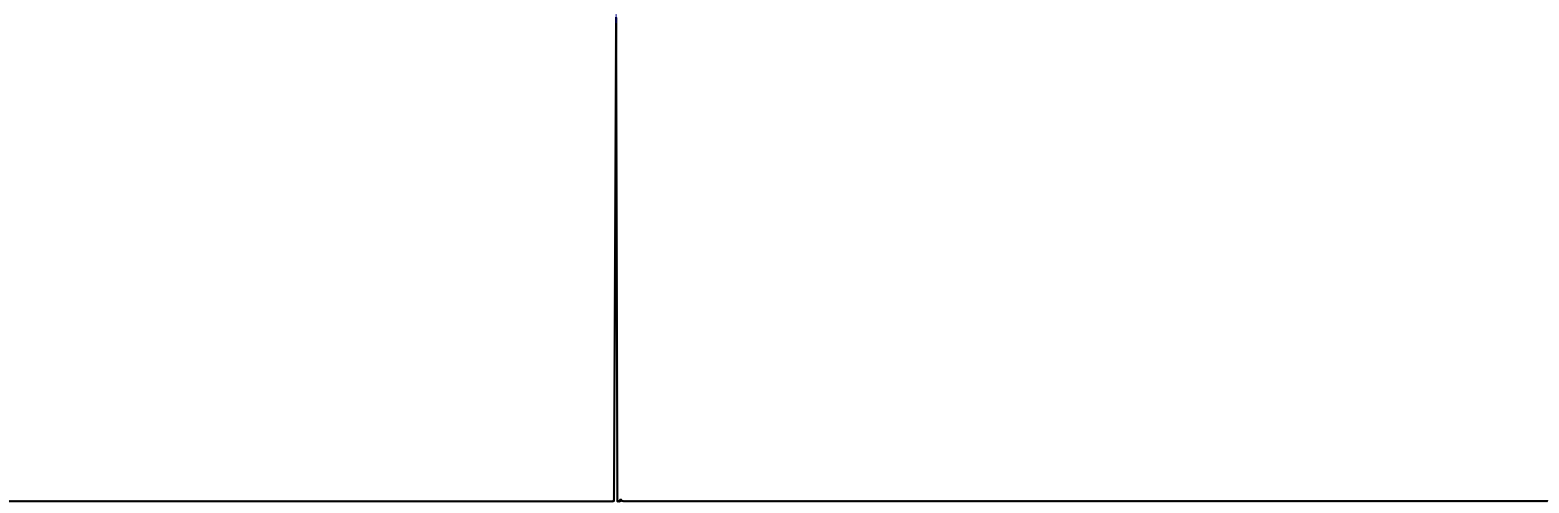

o

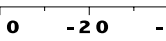

$-30 \quad-40$

$-40-50 \quad-60$ f1 $\stackrel{-80}{(\mathrm{PPm})}$ 
<smiles>Cn1c(=O)c2cc3ccccc3cc2c2c(N3CCOCC3)cccc21</smiles>

3k
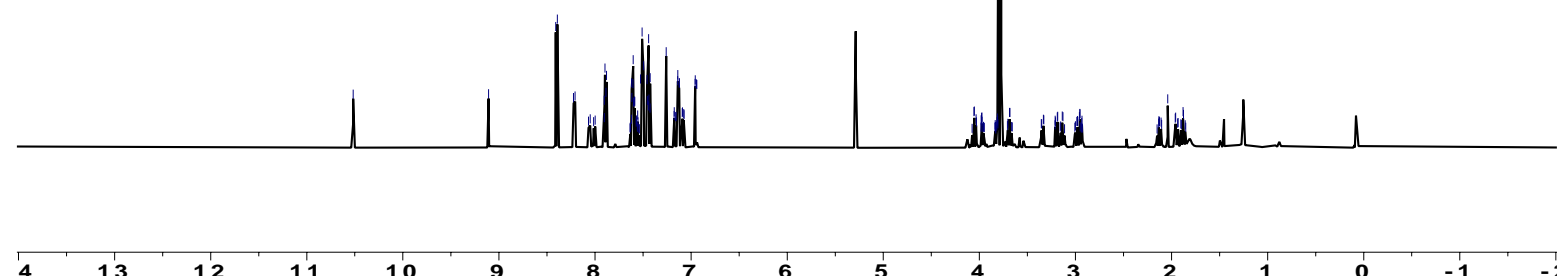

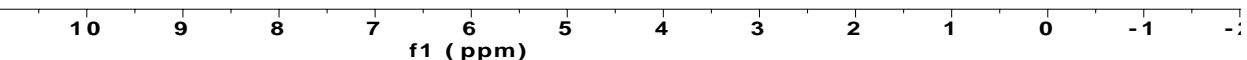
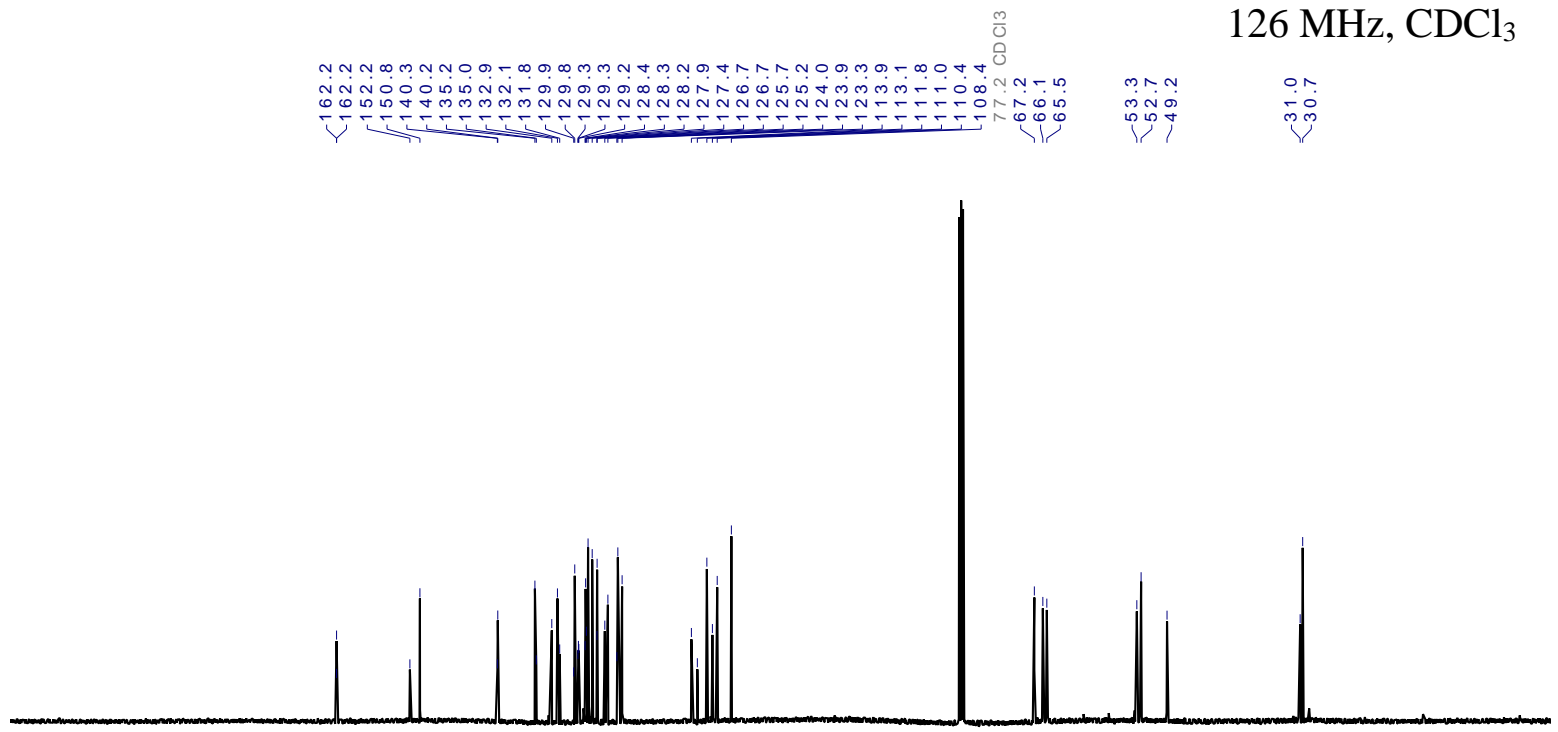

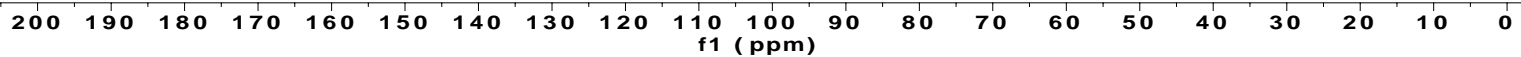




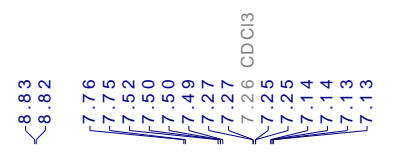

$500 \mathrm{MHz}, \mathrm{CDCl}_{3}$

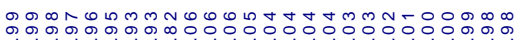

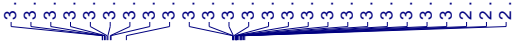<smiles>Cn1c(=O)c2sccc2c2c(N3CCOCC3)cccc21</smiles>

3I

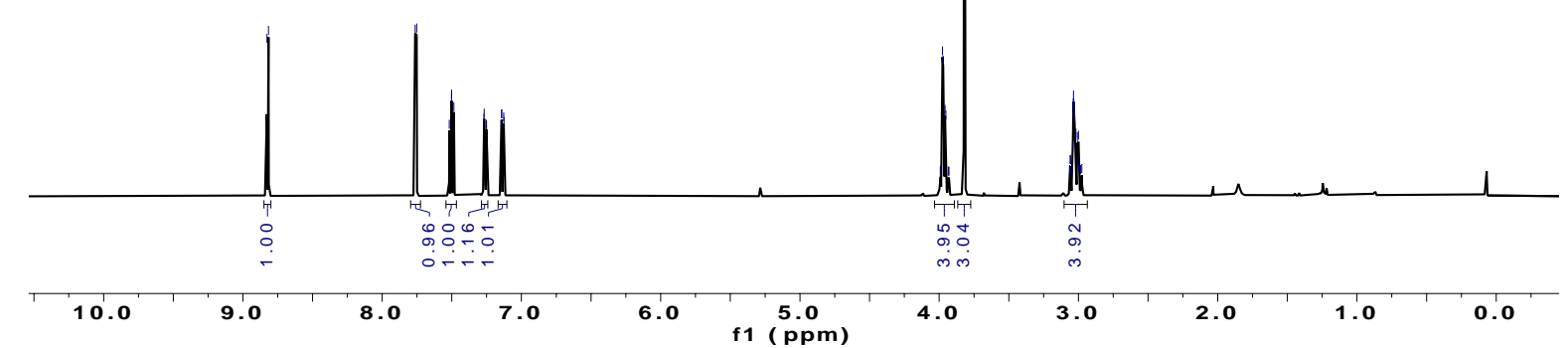

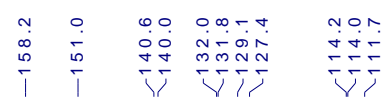

$126 \mathrm{MHz}, \mathrm{CDCl}_{3}$

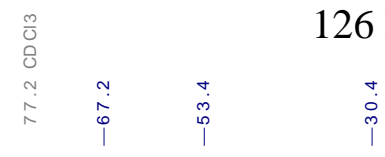

+
0
0

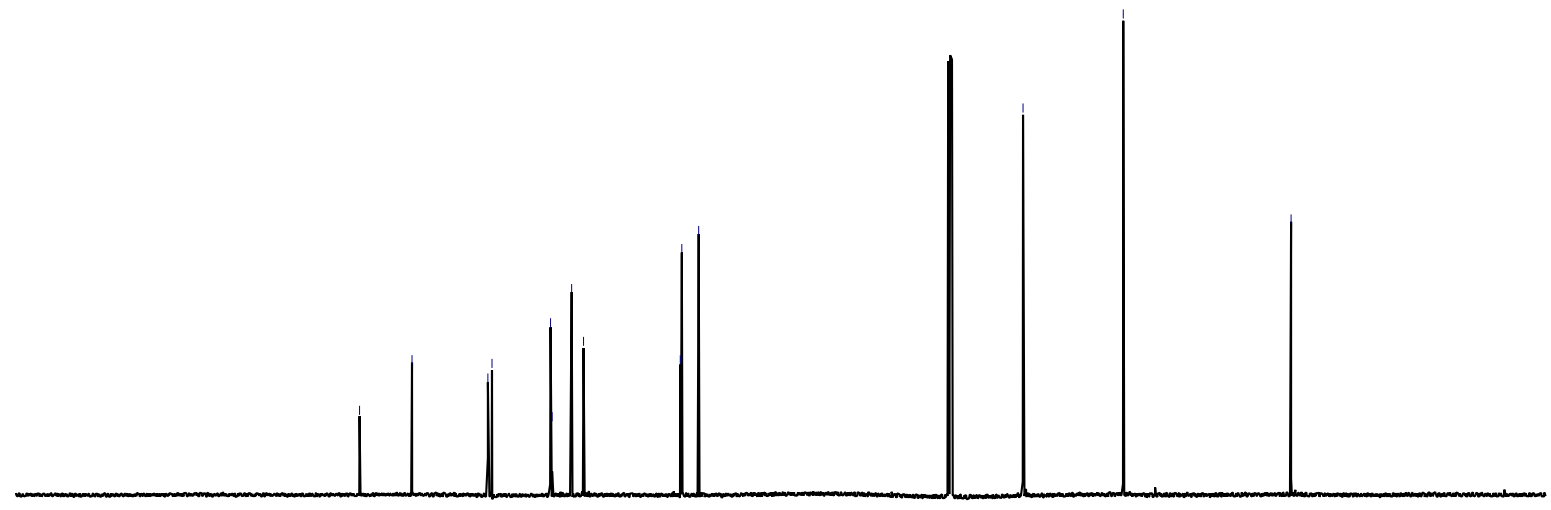

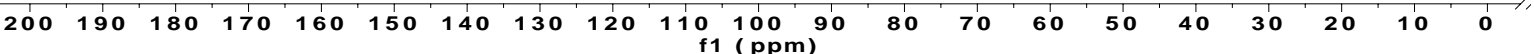




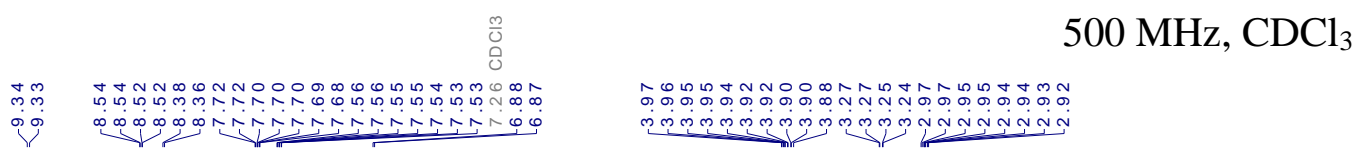<smiles>Cn1c(=O)c2ccccc2c2c(N3CCOCC3)ccnc21</smiles>

$3 \mathrm{~m}$
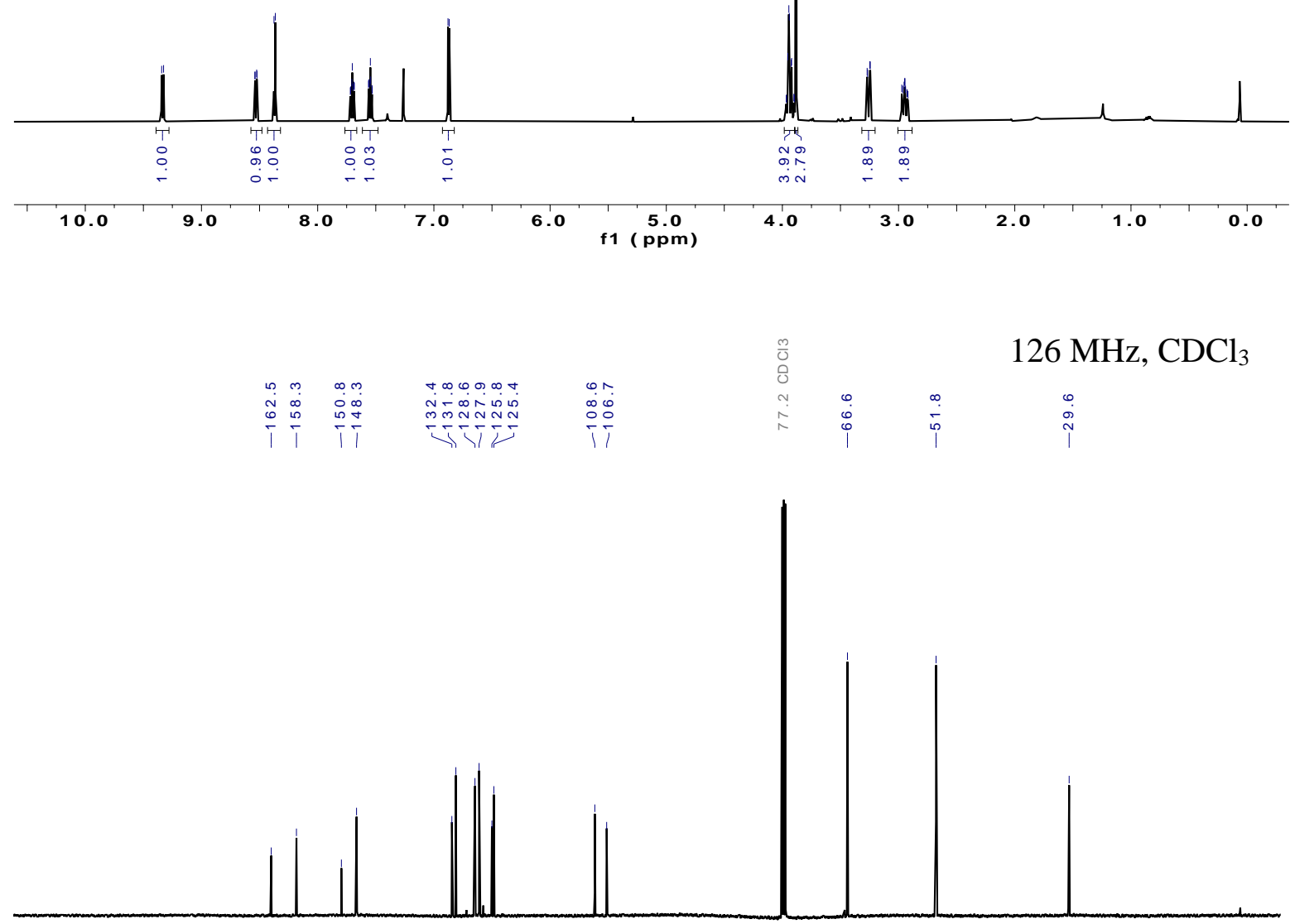

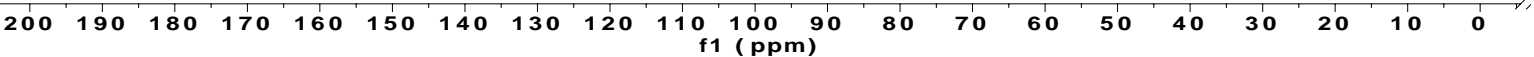






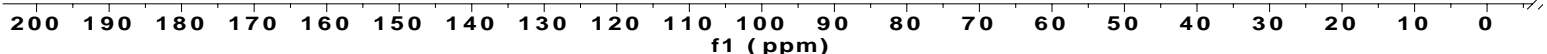



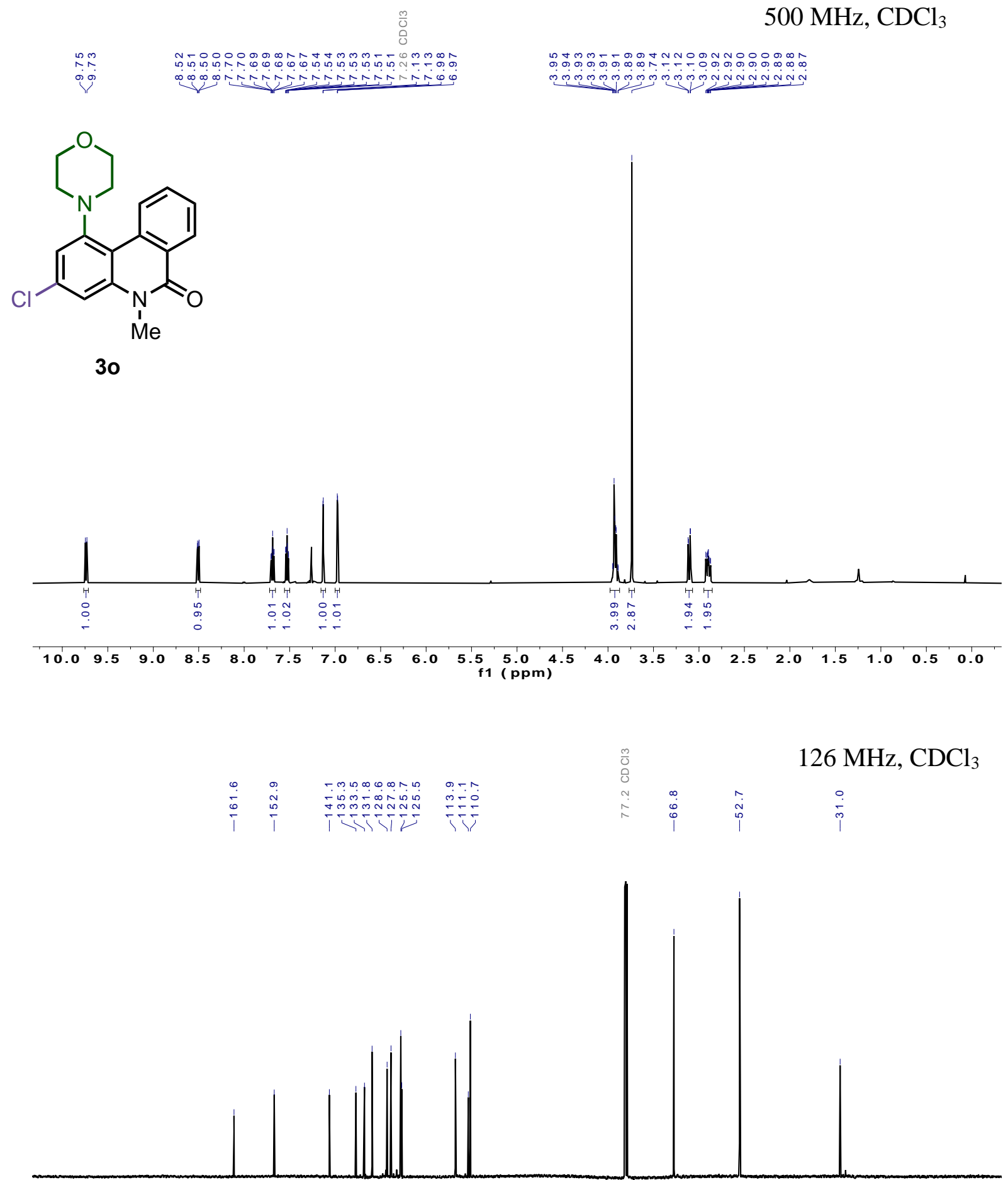

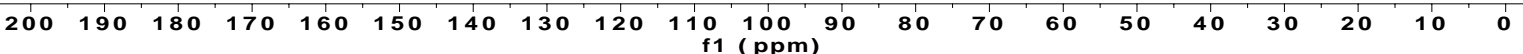



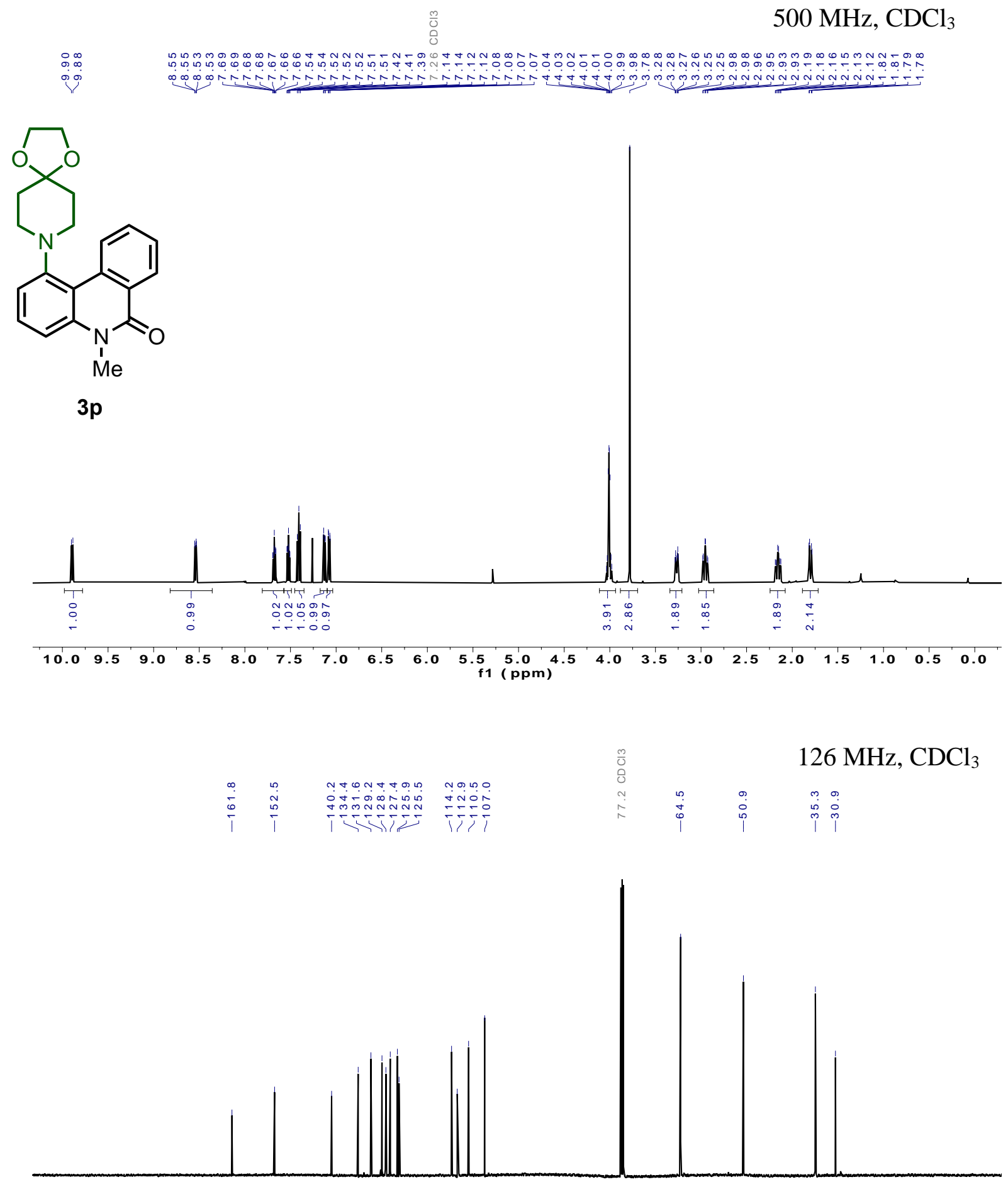

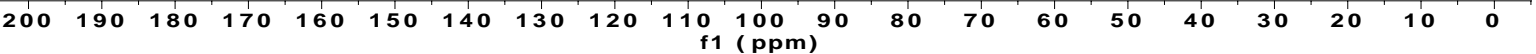


<smiles>Cn1c(=O)c2ccccc2c2c(N3CCN(C(=O)OCc4ccccc4)CC3)cccc21</smiles>

$3 q$
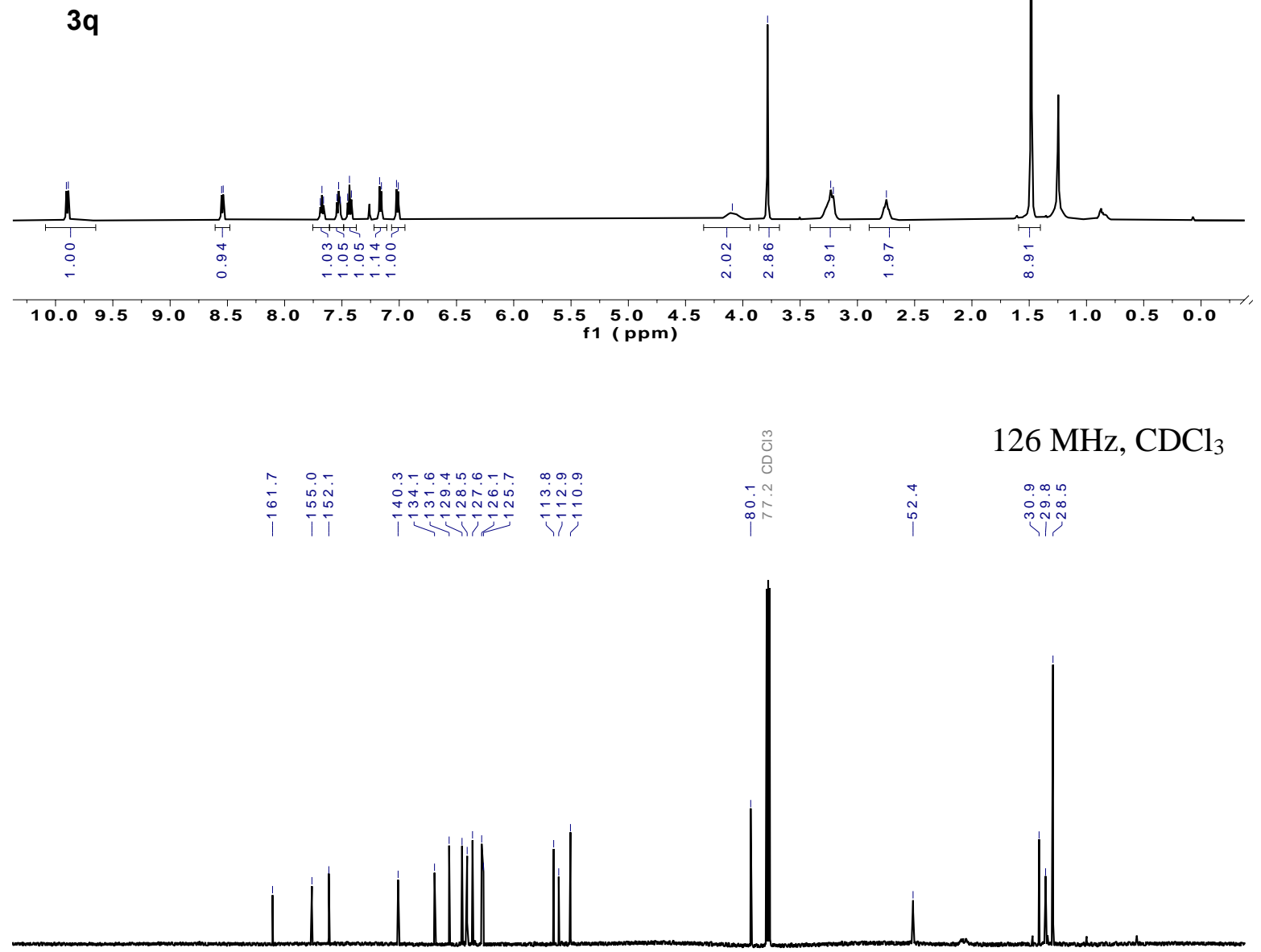

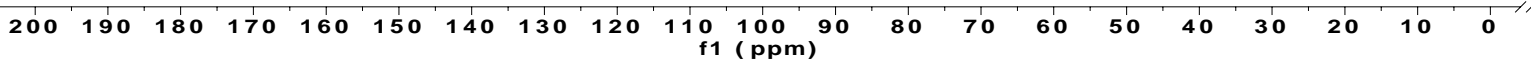




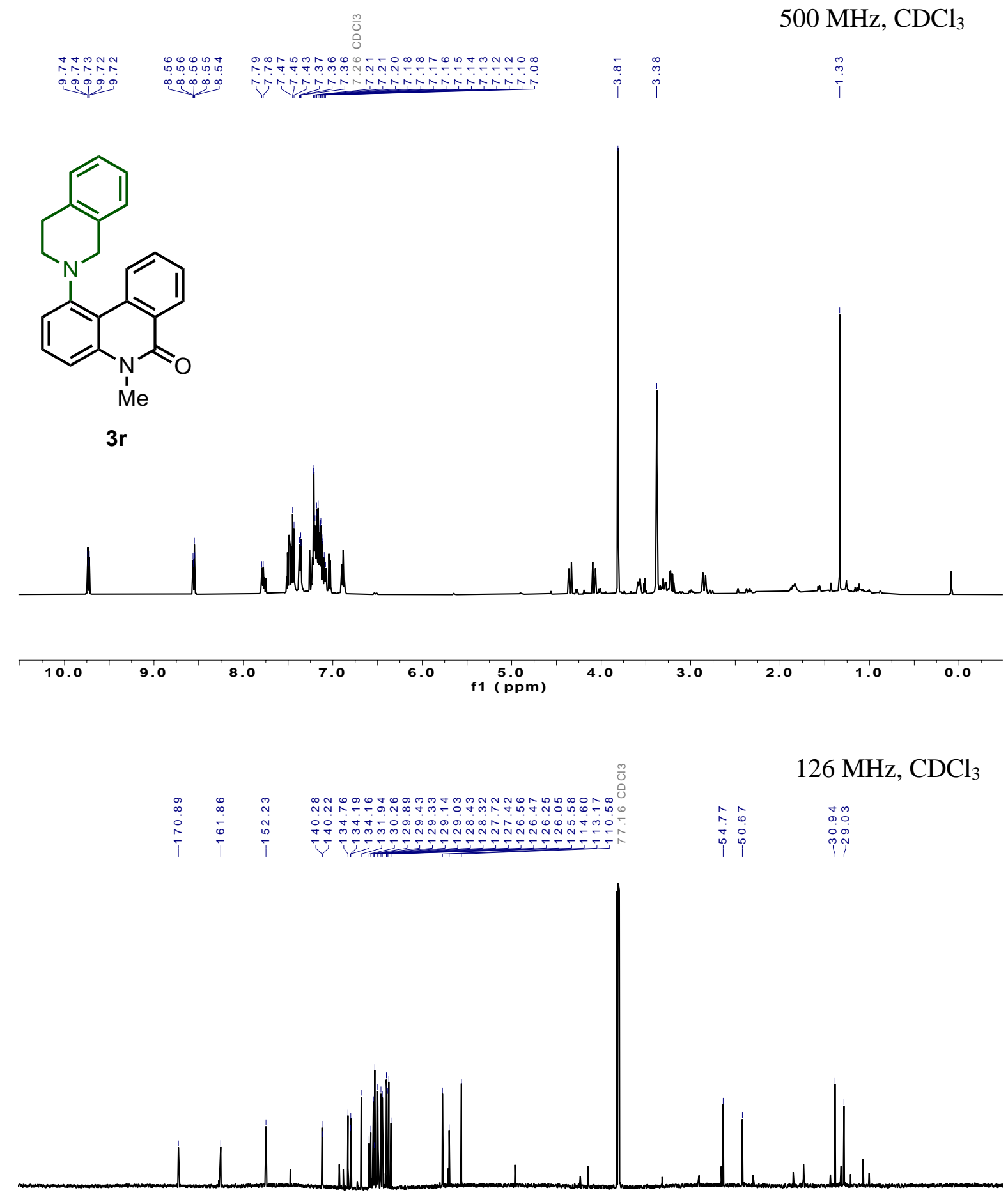

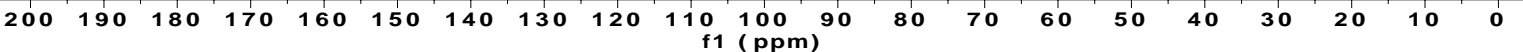




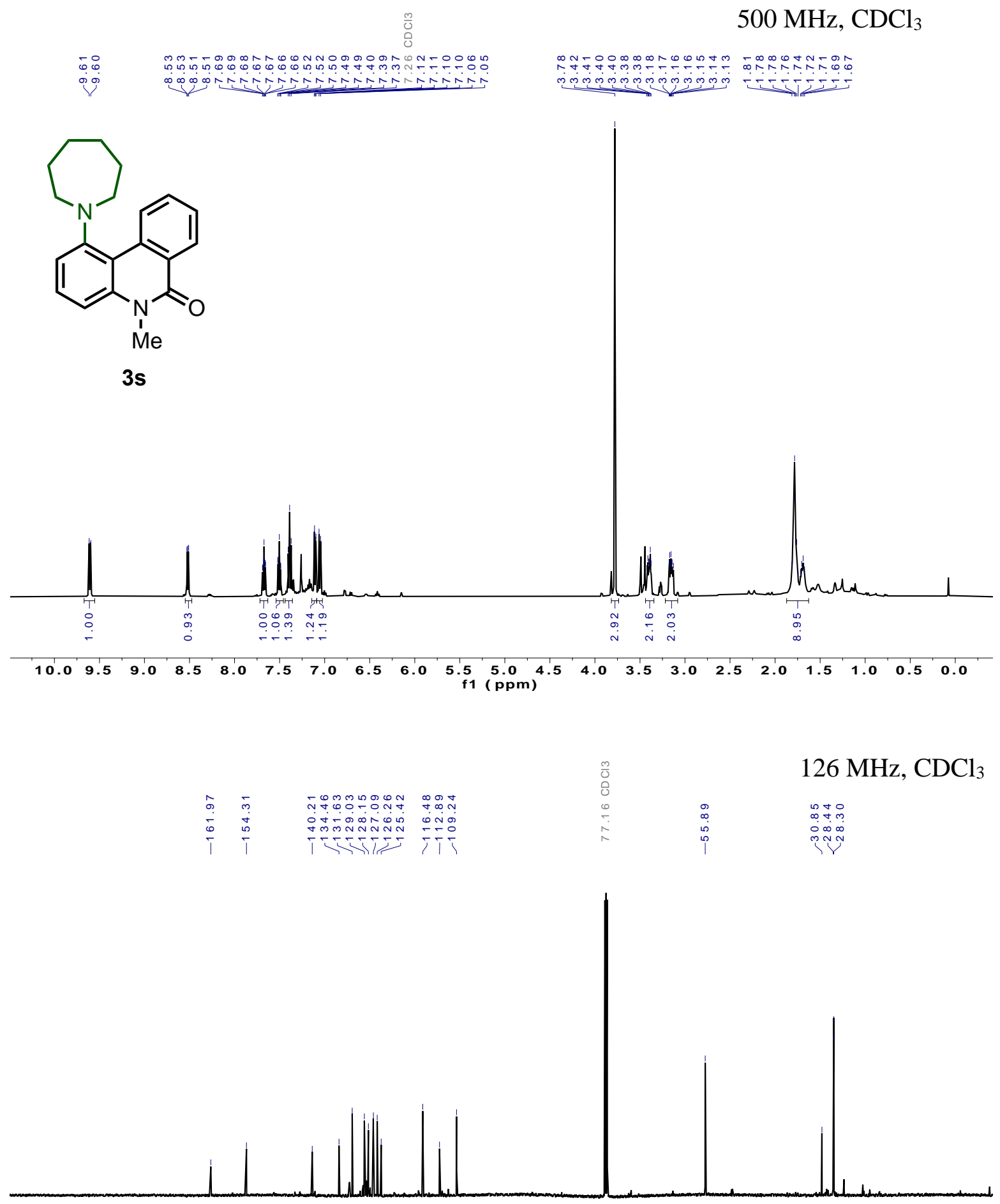

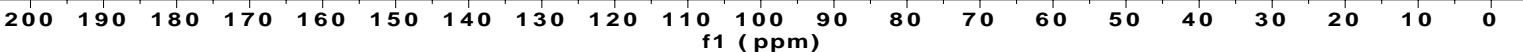




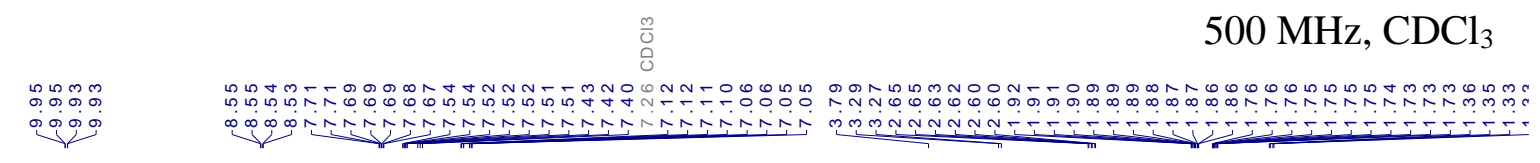<smiles>Cn1c(=O)c2ccccc2c2c(N3CCCCC3)cccc21</smiles>

3t

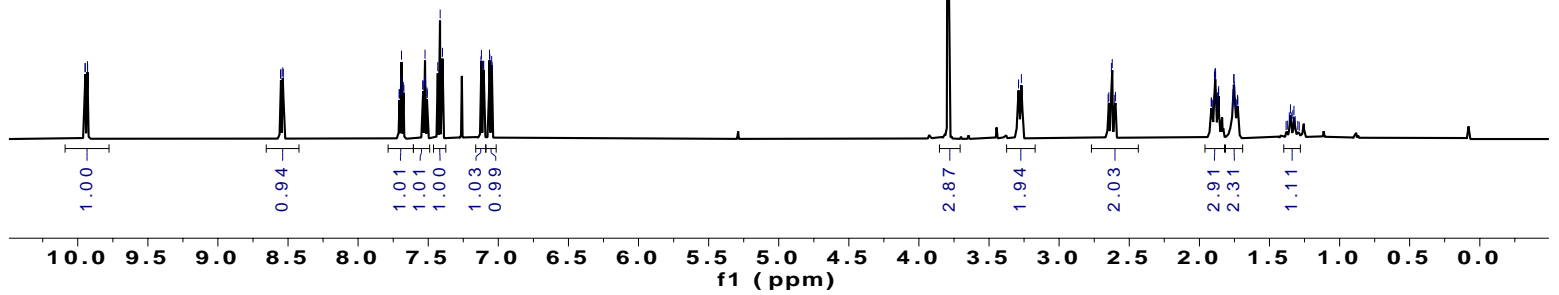

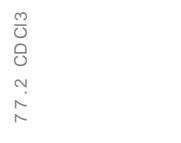

\begin{tabular}{ll}
1 & 1 \\
\hline & 01
\end{tabular}

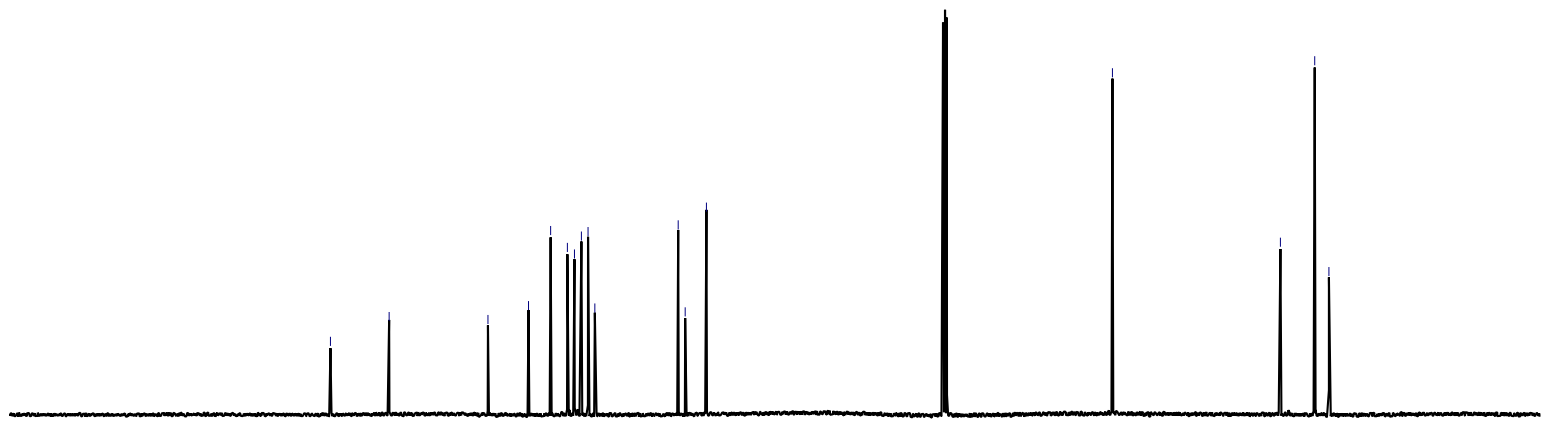

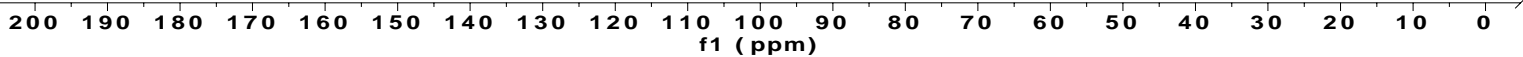




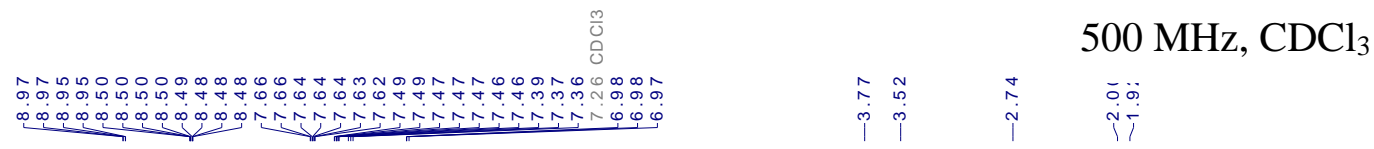<smiles>Cn1c(=O)c2ccccc2c2c(N3CCCC3)cccc21</smiles>

$3 u$
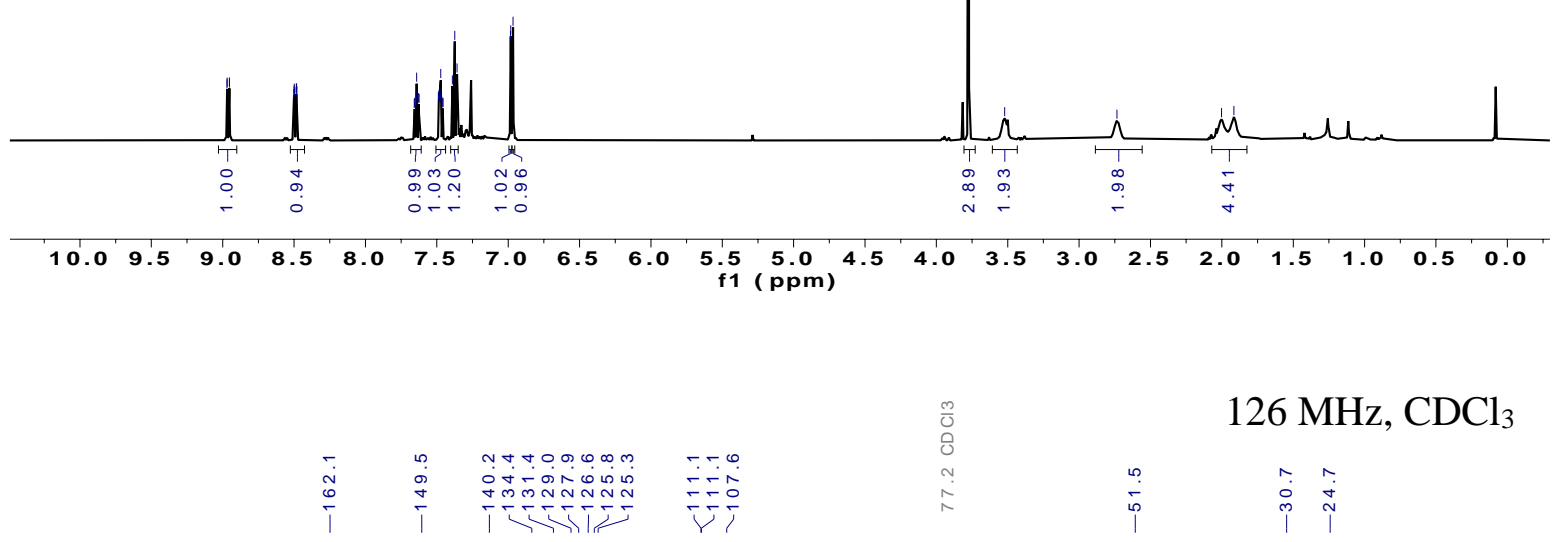

$126 \mathrm{MHz}, \mathrm{CDCl}_{3}$

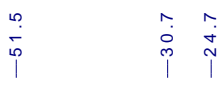

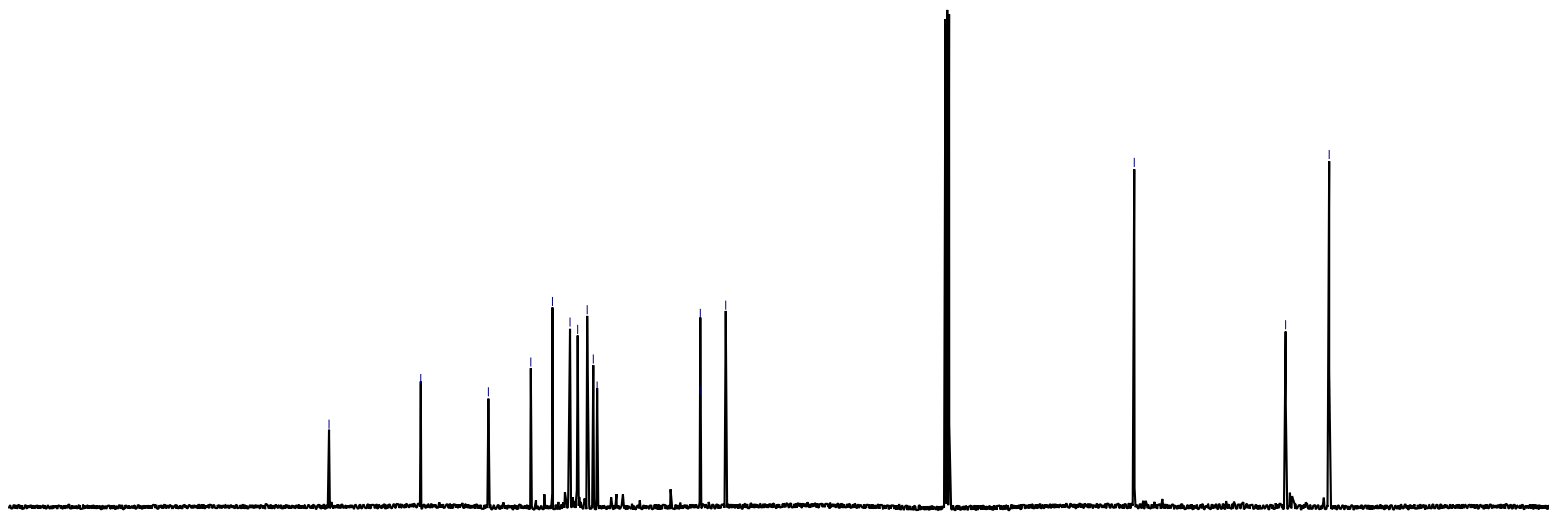

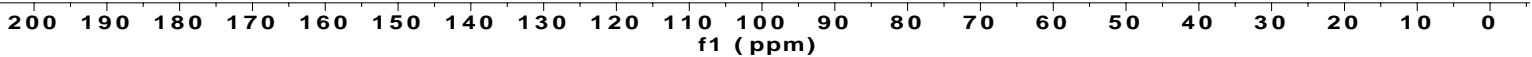




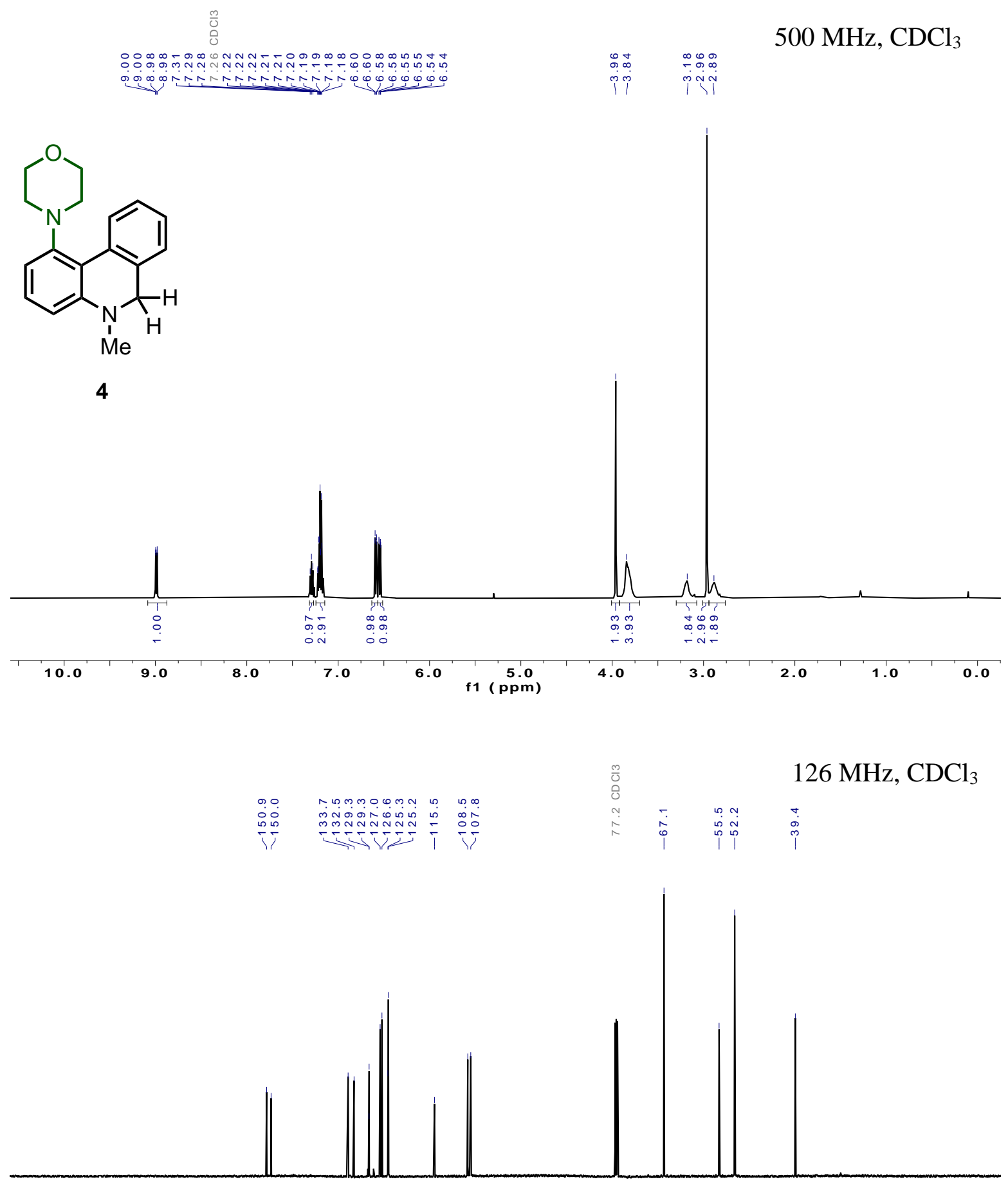

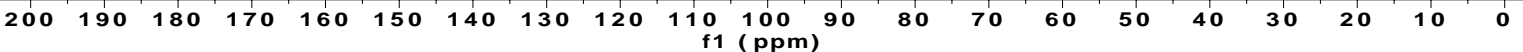




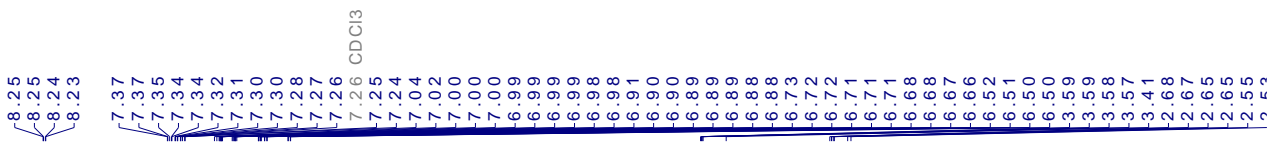

(c)

$6 a$

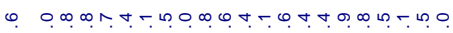

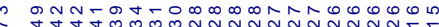

i
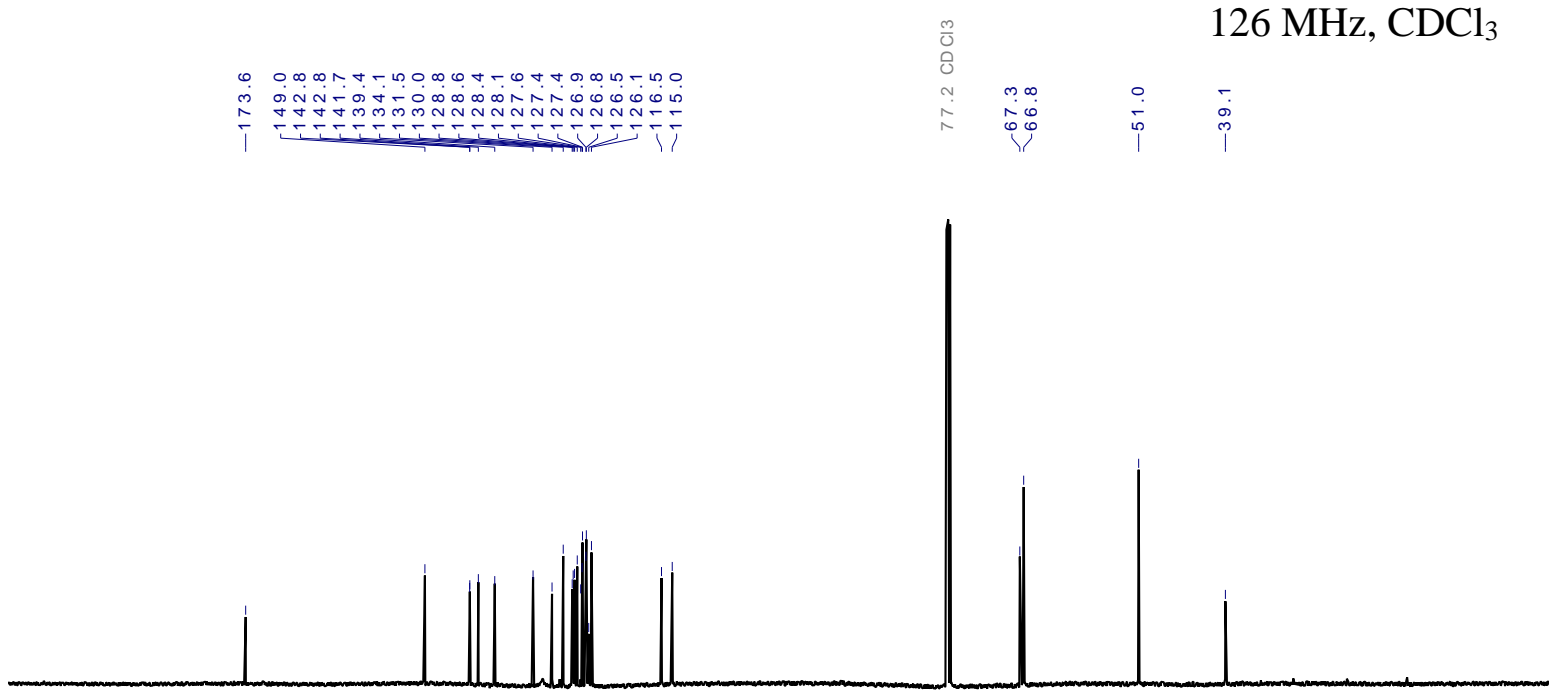

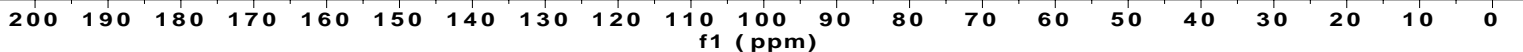




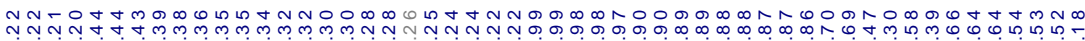

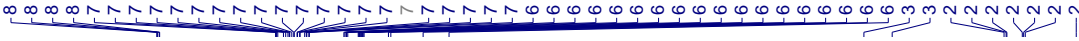<smiles>Cc1cc(N2CCOCC2)c2c(c1)N(C)C(=O)C(c1ccccc1)(c1ccccc1)c1ccccc1-2</smiles>
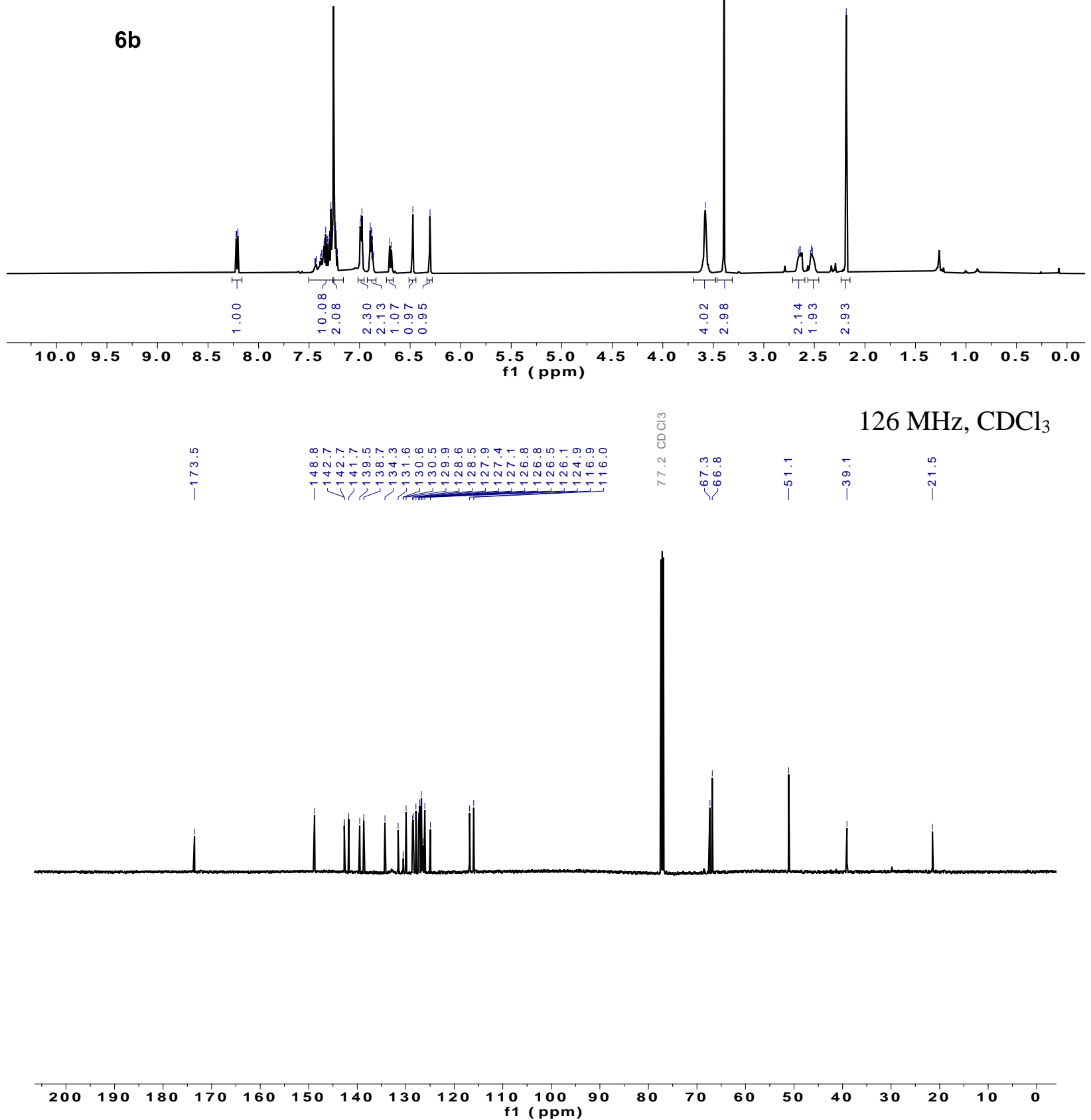
<smiles>Cn1c(=O)c2cccc(N3CCOCC3)c2c2ccccc21</smiles>

8
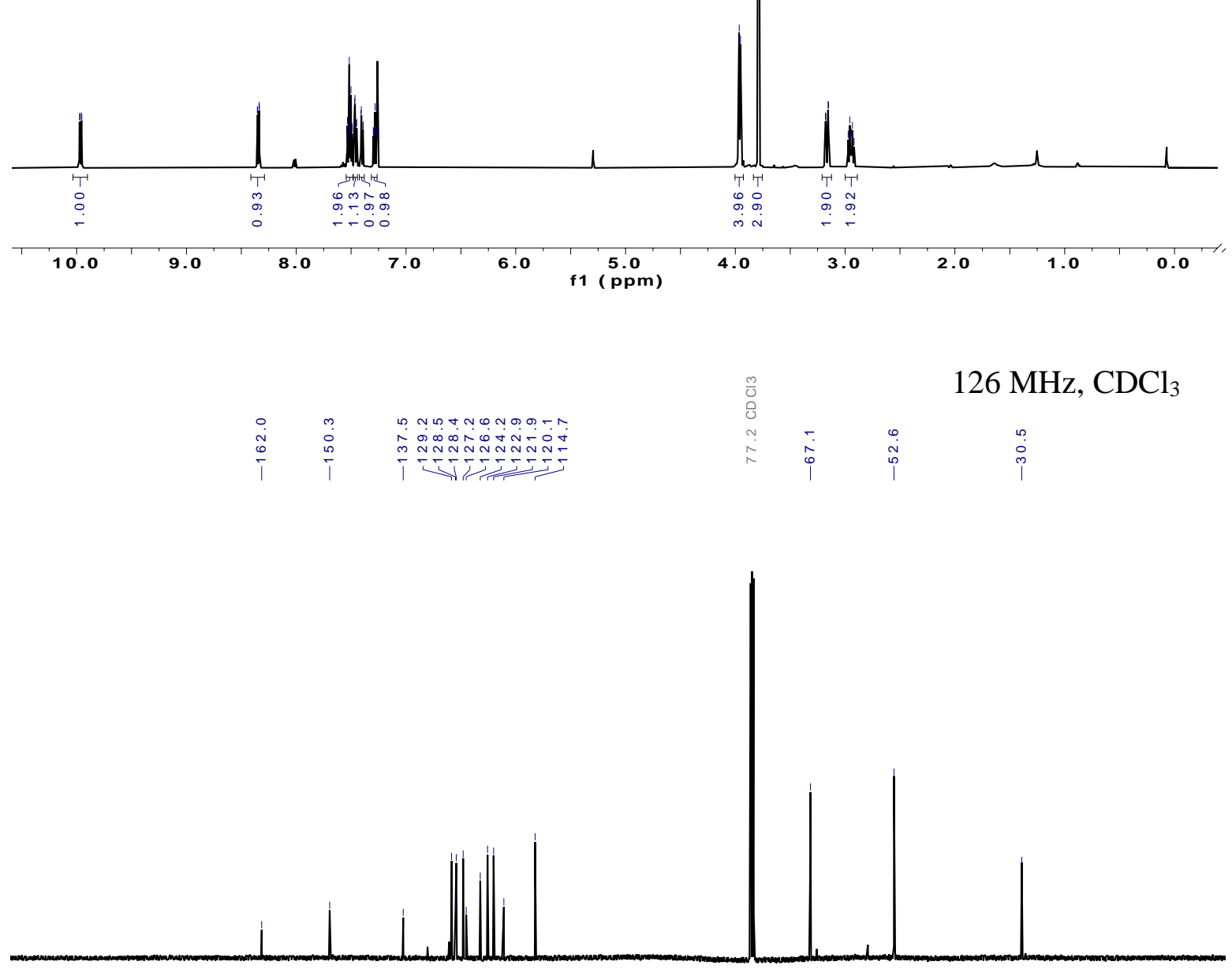

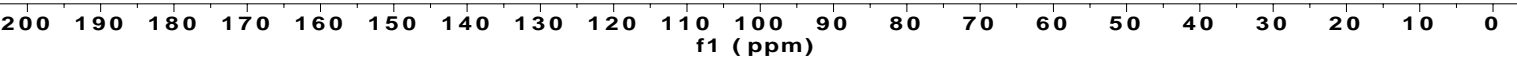




\section{References}

1. Berman, A. M.; Johnson, J. S., Copper-Catalyzed Electrophilic Amination of Organozinc Nucleophiles: Documentation of O-Benzoyl Hydroxylamines as Broadly Useful R2N(+) and RHN(+) Synthons. J. Org. Chem. 2006, 71, 219-224.

2. Zhang, Z.; Liu, Y.-H.; Zhang, X.; Wang, X.-C., KMnO4-mediated oxidative CN bond cleavage of tertiary amines: Synthesis of amides and sulfonamides. Tetrahedron 2019, 75, 27632770 .

3. Roman, D. S.; Takahashi, Y.; Charette, A. B., Potassium tert-Butoxide Promoted Intramolecular Arylation via a Radical Pathway. Org. Lett. 2011, 13, 3242-3245.

4. Iwasaki, H.; Eguchi, T.; Tsutsui, N.; Ohno, H.; Tanaka, T., Samarium(II)-Mediated Spirocyclization by Intramolecular Aryl Radical Addition onto an Aromatic Ring. J. Org. Chem. 2008, 73, 7145-7152.

5. González-López de Turiso, F.; Curran, D. P., Radical Cyclization Approach to Spirocyclohexadienones. Org. Lett. 2005, 7, 151-154.

6. Beccalli, E. M.; Broggini, G.; Martinelli, M.; Paladino, G.; Zoni, C., Synthesis of Tricyclic Quinolones and Naphthyridones by Intramolecular Heck Cyclization of Functionalized ElectronRich Heterocycles. Eur. J. Org. Chem. 2005, 2005, 2091-2096.

7. Matsumoto, S.; Takada, D.; Kageyama, H.; Akazome, M., Formation of benzo[c]thiophen1-aminium iodide by the reaction of o-alkynylbenzothioamide with iodine. Tetrahedron Lett. 2014, 55, 1082-1085.

8. Oda, K.; Ohnuma, T.; Ban, Y., A facile removal of the arenesulfonyl group by electrochemical reduction of sulfonamides in a new cooperative system of anthracene and ascorbic acid: the control of crisscross annulation. J. Org. Chem. 1984, 49, 953-959.

9. An, Y.; Zhang, B.-S.; Zhang, Z.; Liu, C.; Gou, X.-Y.; Ding, Y.-N.; Liang, Y.-M., A carboxylate-assisted amination/unactivated $\mathrm{C}(\mathrm{sp} 2)-\mathrm{H}$ arylation reaction via a palladium/norbornene cooperative catalysis. Chem. Comm. 2020, 56, 5933-5936. 